UCRL-53271

Distribution Category UC- 21

\title{
fotges
}

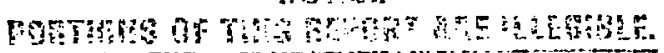

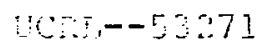

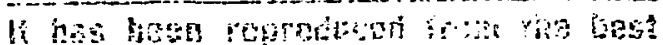

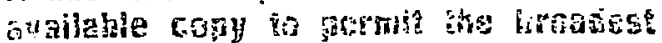

$-2-2 \quad 179$ fossible suabianiby ONW

\section{Time-Resolved Soft-X-ray Studies of Energy Transport in Layered and Planar Laser-Driven Targets}

\author{
Gary Lane Stradling
}

Ph. D. Thesis

Manuscript Date: April 19, 1982

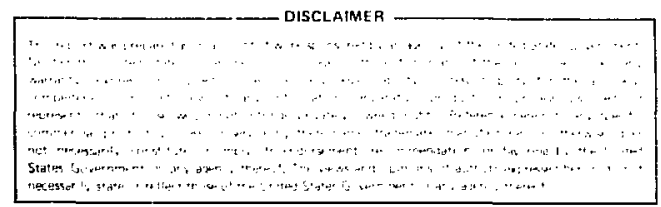

\section{LAWRENCE LIVERMORE LABORATORY University of California • Livermore, California • 94550}


Time Resolved Soft X-ray Studies of Energy Transport in Layered and Planar Laser-Driven Targets

\section{By}

\section{GARY LANE STRADLING}

B.S. (Brighan Young University) 1976

M.S. (University of California, Davis) 1979

M.S. (Brigham Young University) 1981

\section{DISSERTATION}

Submitted in partial satisfaction of the requirements for the degree of DOCTOR OF PHILOSOPHY

in

Engineering-Applied Science

in the

GRADUATE DIVISION

of the

\section{UNIVERSITY OF CALIFORNIA}

\section{DAVIS}

Approved:

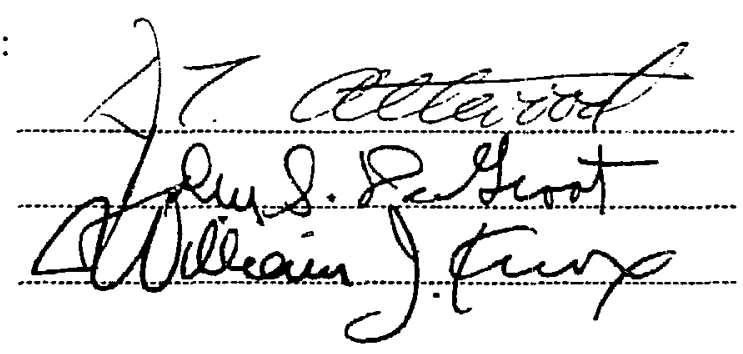

Committee in Charge

Deposited in the University Library 
TABLE OF CONTENTS

ABSTRACT. . . . . . . . . . . . . . . . . . . . . vi vi

ACKNOWLEDGEMENTS ANII DEIJICATION . . . . . . . . . . . . . . . . viii

LIST OF FIGURES . . . . . . . . . . . . . . . . . . . . . . . $x$

CHAPTER

1. INTRODUCTION - THE PROBLE: O OF TEMPORALLy RESOJlVEd LOW-

ENERGY X-RAY AND ENERGY-TRANSPORT DIAGNOSIS IN LASER-

HEATED PLASMAS. . . . . . . . . . . . . . . . . . . . . 1

Introduction. . . . . . . . . . . . . . . 1

Laser Plasma Investigations . . . . . . . . . . . l l

Low-Energy $\mathrm{X}$-Ray Enission . . . . . . . . . . . 2

Soft X-Ray Streak Camera (Chapters 2, 4)....... . 3

Spectral Resolution (Chapter 3)........... . 4

Thermal-Electron Energy Transport (Chapter 5) . . . 4

Sumnary . . . . . . . . . . . . . . . 5

References. . . . . . . . . . . . . . 6

2. NOVEL INSTRUMENTATION FOR OBSERVING X-RAY SIGNATURES OF

ENERGY TRANSPORT IN HIGH-INTENSITY LASER-MATTER

INTERACTION EXPERIMENTS . . . . . . . . . . . . . . . 7

Introduction. . . . . . . . . . . . . . 7

The Soft X-Ray Streak Camera. . . . . . . . . . 7

Concept. . . . . . . . . . . . . . . 7

Soft X-Ray Streak Camera Operation . . . . . 10

The photon detector.......... 10

Electron imaging and streaking. . . . . . 12

Signal amplification. . . . . . . . . 15

Data recording. . . . . . . . . . 15

System Resolution. . . . . . . . . . . 17

References................ . . . 22 
3. SPECTRAL DISCRIMINATION TECHNIQUES: ABSORPTION-EDGE

FILTERING, TOTAL EXTERNAL REFLECTION, AND BRAGG

REFLECTION With METAL MULTtlayers . . . . . . . . . . 24

Introduction. . . . . . . . . . . . . . 24

Absorption-Edge Filtering . . . . . . . . . . 25

Total External X-Ray Reflection . . . . . . . . 26

Theory . . . . . . . . . . . . . 26

Implementation of Reflector/Filter Spectral

Channels.............. 29

Metal-Multilayer X-Ray Interference Mirrors . . . . 32

Advantages and Characteristics . . . . . . 35

Spectrometer Design. . . . . . . . . . 38

Surface reflection. .......... . 38

Metal-Multilayer design........ 39

Metal-Multilayer Calibration . . . . . . 42

Channel Response Calculation......... 42

Spectrometer Assembly and Alignment. . . . . 43

Summary .................... . . 4 4

References. . . . . . . . . . . . . . 48

4. DYNAMIC SOFT-X-RAY STREAK-CAMERA AND PHOTOCATHODE

CHARACTERIZATION : SWEEP-SPEED, SWEEP LINEARITY,

DYNAMIC RANGE, CsI AND Au PHOTOCATHODES . . . . . . . 50

Introduction. . . . . . . . . . . . 50

Pulsed X-Ray Source: The Monojoule-Laser Irradiation Lab. . . . . . . . . . . . . . . 50

Temporal Calibration. . . . . . . . . . . . 54

Evaluation and Comparison of $A U$ and $\mathrm{CsI}$

Transmission Photocathodes . . . . . . . . 57

Side-by-Side Comparisons . . . . . . . . 60 
Temporal Characteristics.... . . . . . 62

References. . . . . . . . . . . . . 67

5. ELECTRON ENERGY-TRANSFORT EXPERIMENTS USTNG LAYERED-

AND SOLID-DISK GEOMETRIES . . . . . . . . . . . . . 68

Introduction. . . . . . . . . . . . . 68

The Experimental Approach . . . . . . . . . . 69

The Experimental Configuration . . . . . . . 70

Summary of Results.... . . . . . . . 74

Solid Disk Results. .. ....... . 74

Layered Disk Results. . . . . . . . . 74

Energy Transport. . . . . . . . . . . . 75

Interesting Experimental Designs. . . . . . . . . 77

Analysis.............. . . . . 82

LASNEX Simulations. . . . . . . . . . . . . 85

Energy Transport in Solid Disks as a Function

of Target Z................. . 88

Experimental Conditions. . . . . . . . . 88

Temporal-Emission Pulse-Shape Comparisons. . . 89

Au disk pulse-shapes at three laser intensities. . . . . . . . . . 90

Pulse-shape differences from disks of varying $Z^{\prime}$ s. . . . . . . . . . 91

Summaries of Pulse-Width Dependencies on

Laser Intensity and Target Z. . . . . . .

Summary of the variation with intensity of Au temporal pulse-width ...... 100

Summary of temporal pulse-width variations

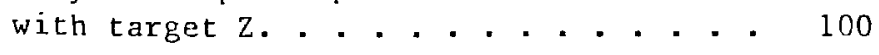

Spectral Data................. 103

Peak Emission Versus $\mathrm{Z}$ and $\mathrm{I}_{\text {laser. . . . . . }} 105$

Layered Disk Energy Transport Experiments . . . . 105 
Temporal Data Comparisons. . . . . . . 107

Peak Flux Comparisons. . . . . . . . . 111

Section Sumary. . . . . . . . . . . 113

Conclusion. . . . . . . . . . . . . . . 114

References.................. 116

APPENDIX

STREAK CAMERA DATA HANDLING, REDUCTION AND ANALYSis. . . 120

Introduction. . . . . . . . . . . . 120

Film Sensitometry.............. . 120

Sensitometer Configuration . . . . . . . 120

Film Processing. . . . . . . . . . . 121

Computer-Controlled Densitometry. . . . . . . . 121

Computer Image Processing and Noise Suppression . . . 122

D-vs-log,i Polynomial Fit . . . . . . . . 122

Streak Data Display. . . . . . . . . . 123

Row and Column Averaging . . . . . . . . 124

Data Smoothing . . . . . . . . . . 125

References. . . . . . . . . . . . . . 128 
Gary Lane Stradling Doctoral Dissertation June, 1982

Englneering-Applied Science University of California-Davis

TIME RESOLVED SOFT $X$-RAY STUDIES OF FNERGY TRANSPORT IN LAYERED

AND PLANAR LASER-DRIVEN TARGETS

\section{ABSTRACT}

New low-energy x-ray diagnostic techniques are used to explore energy-transport processes in laser heated plasmas.

Streak cameras are used for the first time to provide 15-psec time-resolution measurements of subkeV x-ray emission. A very thin $\left(50 \mu \mathrm{g} / \mathrm{cm}^{2}\right)$ carbon subsirate provides a low-energy $\mathrm{x}$-ray transparent window to the transmission photorathode of this soft $x$-ray streak camera. Active differential vacuum puinping of the instrument is required.

$X$-ray spectral resolution, in the region below $1 \mathrm{keV}$ which includes the spectral peak, is obtained through this initial use of the high-energy cutoff properties of $x$-ray reflectors with x-ray streak cameras. In this application absorption-edge filters provide spectral channel definition.

Enhanced spectral resolution in five subkeV spectral bands, 10-eV wide, are made possible through the use of state-of-the-art metal-multilayer $x$-ray interference mirrors. These large d-spacing Bragg reflectors, which are synthesized elsewhere, provide great flexibility in subkeV phocon energy-band selection and detector geometry. High peak reflectivity (10\%) and intermediate bandwidths 
( $10 \mathrm{eV})$ were obtained in this first low-energy application of these structures.

The first use of high-sensitivity, low secondary-electron energyspread CsI photocathodes in x-ray streak cameras is also described. Significant increases in sensitivity with only a small and intermittant decrease in dynamic range were observed.

These coherent, complomentary advances in subkev, time-resolved x-ray diagnostic capability are asplied to energy-transport investigations of $1.06-\mu m$ laser plasmas. Both solid disk targets of a variety of $Z^{\prime}$ s as well as Be-on-Al layered-disk targets were irradiated with 700-psec laser pulses of selected intensity between $3 \times 10^{14}$ $\mathrm{W} / \mathrm{cm}^{2}$ and $1 \times 10^{15} \mathrm{~W} / \mathrm{cm}^{2}$. The temporal pulse-shape of the low-energy thermal emission was analyzed and compared with one-dimensioral LASNEX calculational simulations of identical experiments. A number of interesting trends in the temporal data were observed including dependences of the temporal emission pulse width on target $Z$, laser intensity and $x$-ray photon energy. Be-on-Al layered targets did not exhibit a stepped rise in emission as expected. However

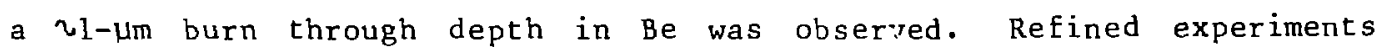
are proposed.

This Dissertation is also Lawrence Livermore National Iaboratory Report 非UCRL-53271 (1982). 


\section{ACKNOWLEDGMENTS AND DEDICATIONS}

A major benefit of this Doctoral program, in addition to the knowledge and handston experience gained, has been the opportunity to work with many excellent scientists and technicians and to learn from their experience.

Dr. David T. Attwood has provided support, motivation, guidance, and much useful insight as my superviser and advisor through these nearly five years. Of particular merit has been his careful effort to insure that I received credit for my contributions. I am grateful for the opportunity to have worked under him.

Dr. John S. DeGroot, my academic advisor at U.C./Davis, has greatly aided my understanding of plasma physics both in class and out.

I have benefited from unrestrained collaborations with both Dr. Burton L. Henke of the University of Hawaii and Dr. Troy W. Barbee, Jr., of Stanford University.

Dr. W. C. Mead has generously provided LASNEX computer simulations for comparison with the time-resolved energy-transport measurements presented here.

Drs. R. L. Kauffman, E. M. Campbell, M. D. Rosen, C. E. Max, W. K. Kruer, and N. M. Ceglio have all shared their insight in numerous useful discussions.

Dr. Hector Medecki has raised my own awareness of ingenious approaches to tough technical problems by his unhesitating and brilliant solutions to such challenges. 
S. W. Thomas, J. W. Houghton, E. L. Pierce, G. E. Phillips, C. H. Dittrure, D. P. Gaines and R. Hockaday have all provided technical assistance which $\mathrm{I}$ wish to acknowledge.

I also thank L. W. Coleman and H. G. Ahlstrom for their programmatic support at Lawrence Livermore National Laboratory as well as R. H. Day and $\mathrm{P}$. B. Lyons for their patience and support in allowing me to finish this Pissertation at Los Alamos National Laboratory.

The most significant support has been provided to me by my wife Rebecca and my children Alden Reid, Suzanne, Seth Gwynn, and Jonathan Gary who have all cheerfully endured many sacrifices of both resources and companionship during my graduate work. I am grateful for their patience and I dedicate this Dissertation to them. 
FICURES

Figure

Page

CHAPTER 2

2-1. X-ray transmission properties of x-ray streak camera photocathode substrates. . . . . . . . . . . . . .

2-2. A cutaway view of the sott $x$-ray streal camera . . . .

2-3. A 10-keV radiograph of the RCA 非73435A (Blank) image converter tube... . . . . . . . . . . . . . . . .

2-4. Microchannel-plate image-intensifier design schemntic. .

\section{CHAPTER 3}

3-1. Reflector/filter channei discriminator design. . . . .

3-2. Efficiencies of three selected $x$-ray reflectors.....

3-3. Three-channel reflector/filter design. . . . . . . . 31

3-4. The three reflector/filter spectral channel shapes

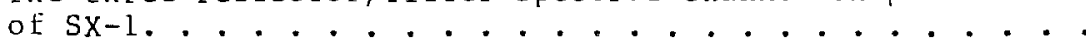

3-5. Schematic configuration of the reflector/filter spectral

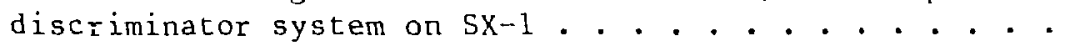

3-6. Constructive interference of $x$-rays, scattered from the periodic multilayer structure, results in Bragg diffraction peaks.....................

3-7. Low-energy surface reflection contributions fiom a metal-multilayer $x$-ray interference mirror may be eliminated using absorption filters. . . . . . . . .

3-8. Five narrow-band metal-multilayer channels for timeresolved subkeV $x$-ray diagnostics. . . . . . . . . . . . 44

3-9. Metal-multilayer spectrometer configuration. . . . . . 45

\section{CHAPTER 4}

4-1. The Monojoule laser, a 50-psec, 1-to-3 joule, neodymium:YAG laser used for diagnostic testing and development. . . . . . . . . . . . . . . 
4-2. The Monojoule target chamber configuration for caibration ald experiments. . . . . . . . . . . .

4-3. Soft x-ray streak camera calibration data. . . . . .

4-4. D-vs-loge characteristic curve of the $\mathrm{film}$, a 9-degree polynomial fit to step-wedge data.......

4-5. An 1ntensity-versus-position scan across the photocathole slit of scft $x$-ray streak camera calibration data. . . . . . . . . . . . . . . .

4-ó. Streaktd intensity-versus-time data obtained using a six-component composite photocathode . . . . .

4-7. Temporal profiles of tr $=1080-\AA \mathrm{CsI}$ and the

$150-\AA$ Au channels of a compesite photocathode response to a train of $270-p s e c x-r a y$ pulses.......

4-8. Relative response-versus-thickness of CsI and Au to ᄂ500-el excitatıon. . . . . . . . . . . . . .

\section{CHAPTER 5}

5-1. The soft $x$-ray streak camera views $x$-ray emission from the heated target through a spectral discrimination apparatus . . . . . . . . . . . . .

5-2. Experimertal geometry of disk target experiments at the Argus las:r facility. . . . . . . . . . . .

5-3. Laser energy is deposiled in the plasma up to critical density $\mathrm{n}_{\mathrm{c}}$. . . . . . . . . . . . . .

5-4. The energy delivered to the target is budgeted inco several distinct categories... . . . . . . . .

5-5. Calculated spatial profiles of the fundamental Be plasma parameters. . . . . . . . . . . . . . .

5-6. Measured and calculated $x$-ray emission temporal pulse-shapes from an Au disk irradiated at $3 \times 10^{13} \mathrm{~W} / \mathrm{cm}^{2}$ laser intensity . . . . . . . . .

5-7. Measirement and calculated $x$-ray emission temporal pulse-shapes from an Au disk irradiated at $3 \times 10^{14} \mathrm{~W} / \mathrm{cm}^{2}$ laser intensity ............ 
5-8. Measured and calculated $x$-ray emission temporal pulse-shapes from an $\mathrm{Au}$ disk irradiated at

$1 \times 10^{15} \mathrm{w} / \mathrm{cm}^{2}$ laser intensity . . . . . . . . . 94

5-9. Measured and calculated $x$-ray emission temporal

pulse-shapes from an Al disk irradiated at

$3 \times 10^{14} \mathrm{~W} / \mathrm{cm}^{2}$ laser intensity . . . . . . . . . \$5

5-10. Measured and calculated $x$-ray emission temporal

pulse-shapes from a Be disk irradiated at

$3 \times 10^{14} \mathrm{~W} / \mathrm{cm}^{2}$ laser intensity............ 95

5-11. The ratio of Au $x-r a y$ emission pulse-widths to

the laser pulse- $h^{-}$th as a function of laser

intensity. . . . . . . . . . . . . . . . . 98

5-12. X-ray emission pulse-widths as a function of

target $\mathrm{Z}_{\mathrm{eff}} \cdot . \cdot \cdot \cdot \cdot \cdot \cdot \cdot \cdot \cdot \cdot \cdot \cdot \cdot \cdot \cdot \cdot \cdot \cdot \cdot \cdot \cdot 99$

5-13. The peak $x$-ray emission level in the 200-eV

carbon channel from $S X-1$ is seen to vary nearly

linearly with the $z_{\text {eff }}$ of the emitting plasma. . . . . 102

5-14. Absolute Au spectral data from the reflector/

filter system $(S X-1)$, from the metal-multilayer

$x$-ray-interference-mirror system $(S X-6)$ and from

LASNEX calculations at $3 \times 10^{13} \mathrm{~W} / \mathrm{cm}^{2}$,

$3 \times 10^{14} \mathrm{~W} / \mathrm{cm}^{2}$, and $1 \times 10^{15} \mathrm{~W} / \mathrm{cm}^{2}$ laser

intensities. . . . . . . . . . . . . . . .

5-15. Absolute values of peak flux from $\mathrm{Au}, \mathrm{Ti}$, and

Be disks as a function of laser intensity on target. . . . 106

5-16. X-ray emission histories, at $200 \mathrm{eV}$, from

layered Be-on-Al disk targets. . . . . . . . . . 108

5-17. X-ray emission histories, at $700 \mathrm{eV}$, from

layered Be-on-A1 argets . . . . . . . . . . . .

5-18. Peak $x$-ray flux as a function of Be coating

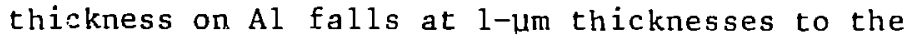

level of solid Be experiments. . . . . . . . . .

\section{APPENDTX}

A-1. Streaked image of an $x$-ray pulse from an Au disk

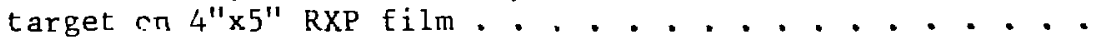

A-2. A seven-degree polynomial fit to the exposure step-

wedge densities. . . . . . . . . . . . . . . . . 
A-3. Density-vs-log exposure polynomial fit to the step

wedge from figure A-2. . . . . . . . . . . . . .

A-4. Iso-density contours of the data of figus $A-1 . . . .$.

A-5. The iso-density contour plot with late-time rows

eliminated and maximum resolution in the time

direction. . . . . . . . . . . . . . . . .

A-6. The previous figure converted to iso-intensity

contours........................

A-7. The previous figure with rows 70 through 110

designated for time averaging. . . . . . . . . . .

A-8. Time average of intensity values for rows

70 through 110 across the spatial extent of

the photocathode . . . . . . . . . . . . . .

A-9. Figure A-6 with the extent of the three channels

to be spatially averaged marked. . . . . . . . . .

A-10. The average value of the carbon filtered channel

as a function of time over $3.2 \mathrm{nsec} . .$. . . . . . . .

A-11. The average value of the vanadium filtered channel

as a function of time over $3.2 \mathrm{nsec}$. . . . . . . . .

A-12. The average valie of the iron filtered channel

as a function of time over $3.2 \mathrm{nsec..........}$

A-13. A composite of the three energy channels . . . . . .

A-14. Streaked image of low-level, noisy data from

a Be disk irradiation on $4^{\prime \prime} \times 5^{\prime \prime} \mathrm{film.} \mathrm{.} \mathrm{.} \mathrm{.} \mathrm{.} \mathrm{.} \mathrm{.} \mathrm{.} \mathrm{.} \mathrm{.}$

A-15. Polynomial fit to step wedge of figure A-14........

A-16. Density-vs-log exposure for the film of

figure A-14. . . . . . . . . . . . . . . .

A-17. Iso-density contour piui of the Be-data streaked image from figure A-14................

A-18. Converted iso-intensity contours from figure A-17. . . . . . . . . . . . . . . .

A-19. Iso-intensity contour plot marked for temporal averaging over rows 50 through 140 
A-20. Photocathode-position dependent, temporally-

averaged channel levels............... 148

A-21. Iso-intensity contour plot marked for spatial

averaging in three channels. . . . . . . . . . . . 149

A-22. Spatially averaged 200-eV (carbon filtered)

channel as a function of time. . . .......... 150

A-23. Spatially-averaged 500-eV (vanadium filtered)

channel. . . . . . . . . . . . . . . . 151

A-24. Spatially-averaged 700-eV (iron filtered) channel. . . . 152

A-25. The spalially-averaged 200-eV channel. . . . . . . . 153

A-26. The spatially-averaged $200-e V$ channel with

120-psec temporal smoothing. . . . . . . . . . . . 154

A-27. The spatially-averaged 200-eV channel with

180-psec temporal smoothing. . . . . . . . . . . . 155 
CHAPTER 1

\author{
INTRODUCTION - THE PROBLEM OF TEMPORALLY RESOLVED \\ LOW-ENERGY X-RAY AND ENERGY-TRANSPORT DIAGNOSIS \\ OF LASER $\cdots$ HEATED PLASMAS
}

\title{
Introduction
}

This Dissertation addresses the issues of subkilovolt $x$-ray diagnosis and thermal energy transport in $1.06-\mu m$ laser heated plasmas through the use of novel $x-r a y$ instrumentarion and diagnostic techniques. The instrumentation, including: subkev-sensitive, 15-psec soft $x$-ray streak cameras, reflector/filter (broadband) spectral discrimination, metal-multilayer $x$-ray interference mirror (intermediate resolution) spectral discrimination and high-sensitivity CsI photucathodes, all represent new and relevant contributions to the high temporal resolution, low-energy x-ray diagnostic capability of the laser-plasma community. The energy-transport diagnostic technique of temporally resolving thermal x-ray emission from both layered and homogeneous disk targets is also a new contribution to laser-plasma analysis and should result in improved modeling of the evolution of laser-heated plasmas.

\section{Laser Plasma Investigations}

Laser-heated p.lasinas are extremely complex phenomena. If they are to be understood and used effectively, progress must be made-pushing back the current limits of plasma theory, hydrodynamic calculational modeling, and plasma diagnostics. The processes of importance in such plasmas are interrelated and include: light absorption, electron heating, energy transport, hydrodynamic expansion, electron and ion plasma oscillat:ons, atomic processes, x-ray emission, hydrodynamic motion, and plasma instabilities, and magnetic field generation. In laser fusion related experiments these processes occur with scale lengths of microns to hundreds of microns and overall characteristic times ranging from picoseconds to hundreds of picoseconds. 1 
As with most complicated phenomena, improvements in theoretical and calculational modeling of laser-heated plasmas are compelled by and constrained by diagnostic observations. The more sophisticated, detailed, and reliable the plasma measurements, the more precise the modeling is constrained to be. Improved modeling of the plasma, in turn, directs the experimental efforts to relevant measurements which should provide more useful information.

\section{Low-Energy X-Ray Emission}

Because of the size, time scale, and nonuniformity of laser-heated plasmas, most of the processes mentioned above are not directly accessible for detailed measurement.

One of the most useful observables from $1.06-\mu \mathrm{m}$ laser-driven plasmas is the low-energy x-ray emission. This observable comprises a significant portion of the energy balance in the plasma, it is a diagnostic of the electron density and temperature, and it can provide dynamical information about energy transport in the plasma.

Electron temperature, a very important parameter, is a highly variable function of position and time. To date, the electron temperature is generally construed from coarse spectral-resolution, space- and time-integrated, x-ray emission measurements. Thermal x-ray emission from these 1.06- $\mu \mathrm{m}$ laser-driven plasmas, where the electron temperature in the peak emission region of the target is on the order of $100 \mathrm{eV}$, lies mostly in the photon-energy region below $1 \mathrm{keV}$.

At the time that the research presented in this Dissertation was initiated (1977), temporally resolved capability for measuring 
subkilovolt $x$-ray emission was limited to -200 psec resolution in broad spectral bands with $x$-ray diode (XRD) detectors coupled to fast oscilloscopes. ${ }^{2}$ These quasi-time-integrated measurements provided the amount of energy in overall x-ray emission, as well as a rough spectral shape. Other completely time integrated, high spectral resolution measurements could provide a detailed spectral shape. However, the emission spectrum changes a great deal with time as the plasma rapidly heats and then slowly cools. The instrumentation available then was inadequate to allow the dynamic study of thermal plasina processes such as energy transport.

The first four chapters of this Dissertation describe the extension of $x$-ray diagnostic capabilities to higher temporal resolution, improved spectral resolution, enhanced sensitivity and a spectral sensitivity range down to $100 \mathrm{eV}$. In Chapter 5 these refined capabilities are applied to the problem of evaluating energy transport characteristics of laser plasmas under a variety of conditions. An Appendix follows with a discussion of $x$-ray streak camera data analysis.

\section{Soft X-Ray Streak Camera (Chapters 2,4 )}

Temporal resolution of $\sim 15$ psec and spectral sensitivity down to $100 \mathrm{eV}$ was made available for the first time through the modification of hitherto $>1-\mathrm{keV}$ x-ray streak camera technology. 3 The resulting soft $x$-ray streak camera design included very thin carbon films as photocathode substrates, demountable photocathode assembiies, and a differential, active pumping scheme to maintain the necessary vacuum. The use of streak cameras for fast detectors makes available large 
dynamic range, shot noise limited sensitivity, and a large number of contiguous, synchronized detection channels in addition to 15-psec temporal resolution.

Photocathode evaluations performed with this flexible system established 1100- $\AA$ thick $\mathrm{CsI}$ as a useful, high-sensitivity photocathode for $x$-ray streak cameras.

\section{Spectral Resolution ( $r$ apter 3)}

Broad but discrete spectral channels were obtained in this difficult (subkeV) spectral region, which encompasses the peak of the emission spectrum, using $x$-ray reflectors to cut off high-energy portions of the spectrum passed by absorption-edge filters. This first use of this reflection technique with $x$-ray streak cameras avoids a less precise, iterative procedure in which the subtraction of overlapping spectral channels is usad to obtain broad spectral resolution.

Another increase in spectral resolution of time-resolved subkeV x-ray emissions was first achieved in this work through the use of metal-multilayer $x$-ray interference mirrors. These state-of-the-art, large d-spacing, Bragg reflectors (developed elsewhere) provided intermediate spectral resolution in five $10-\mathrm{eV}$ bands between $100 \mathrm{eV}$ and $1 \mathrm{keV}$.

\section{Thermal-Electron Energy Transport (Chapter 5)}

Detailed thermal-energy transport in layered targets may be dynamically observed using spectrally labeled materials as the various layers. The ability to temporally resolve thermal x-ray emission with 
good spectral resolution allowed this thermal-energy transport diagnostic technique to be implemented for the first time.

Although the detailed experiments were not performed due to laser scheduling constraints, initial efforts with sinpler-than-desired targets are described along with suggestions for more refined experiments.

The simple-target experiments provided a number of useful observations on the t, supcral and spectral $x$-ray emission characteristics both of homogeneous targets of various Z's and of Be-on-Al layered targets. Detailed comparisons are shown with one-dimensional hydrodynamic calculations using a Lagrangian, multigroup, hydrodynamic code LASNEX ${ }^{4,5}$ to simulate the experiments. These comparisons point to further refinements necessary to bring calculational modeling of the experiments closer to the observed phenomona.

\section{$\underline{\text { Summary }}$}

The contributions to laser-plasma investigation described in this Dissertation are a coherent series of technical advances opening the way for refined knowledge of subkeV x-ray emission signatures of both energy-balance and thermal-energy-transport phenomena.

Comparison of the improved diagnostic measurements with state-ofthe-art computational modeling of plasma evolution has identified areas for improvement of the modeling.

A number of areas for further experimental work are discussed. 


\section{References}

1. D. T. Attwood, IEEE, J. Quantum Electron. QE-14, 909 (1978).

2. K. G. Tirsell, H. N. Kornblum, V. W. Slivinsky, Bull. Amer. Phys. Soc. 23, 807 (1978); Lawrence Livermore National Laboratory report, UCRL-81378 (1979, unpublished).

3. C. F. MeConaghy and L. W. Coleman, Appl. Phys. Lett. 25, 268 (1974).

4. W. C. Mead, private communication.

5. G. B. Zimmerman and W. J., Kruer, Comments Plasma Phys. 2, 85 (1975); G. B. Zimnerman, Lawrence Livermore Laboratory report, UCRL-74811 (1973, unpubljshed). 


\title{
Chapter 2
}

\author{
NOVEL INSTRUMENTATION FOR OBSERVING X-RAY SIGNATURES \\ OF ENERGY TRANSPORT IN HIGH-INTENSITY LASER- \\ MATTF.R JNTERACT ION EXPERIMENTS
}

\section{Introduction}

This chapter discusses the temporally and spectrally resolving instrumentation used to observe $x$-ray emission characteristics of laser produced plasmas. Pertinent aspects of soft $x$-ray streak camera (SXRSC) technology, including the photocathode function, the image converter, intensifier, and recording units, and the temporal resolution of the system are described here.

\section{The Soft X-Ray Streak Camera}

\section{Concept}

The soft $x$-ray streak camera, an extension of existing $x$-ray streak camera $^{1,2}$ technology, is an $x$-ray detector with 15-psec time resolution, large dynamic range, subkilovolt sensitivity and synchronous multichannel recording capability. 3,4 High temporal resolution is obtained by converting time-varying $x$-ray photon flux into similarly time-varying space current of secondary electrons emitted from a photocathode. This electron emission is electrically imaged onto a phosphor screen. Rapid deflection of the electron stream displaces the image of the photocathode exission with time. This results in a streaked image with one dimension of temporal information. Early development and application of electro-optical streak cameras in the USSR, England, Canada, and the USA are described in references 5-17. A continuous photocathode provides a dimension of spatial discrimination which is 
used to obtain simultaneous multichannel detection of a spectrally or spatially resolved signal imaged onto the photocathode.

The streak camera is an extremely sensitive, shot noise limited, quantum detectur with large dynamic rangu. "ihe dynamic range is limited by shot-roise statistics at low flux levels. At high flux levels, the dynamic range is limited by the recording medium and by distortions of focusing from space-charge repulsion at the photocathode and in the electron crossover region. ${ }^{18}$ While dynamic range 19 depends on the temporal resolution required, values of $210^{3}$ are obtainable for temporal detail relevant to inertial fusion.

Sensitivity in the subkilovolt portion of the $x$-ray spectrum is obtained by modifying a well-established $x$-ray streak camera design ${ }^{1}$ with a thin, low-Z, soft $x$-ray transmitting window and an active vacuum pumping arrangement. ${ }^{4}$ previous $x$-ray streak camera designs utilized a vacuum-tight beryliium window to transmit $x$-rays of energies greater than a kilovolt (figure 2-1). The original LLNL x-ray streak camera used an Au transmission photocathode vacuum deposited on the inside of the beryllium vacuum window. In the soft $x$-ray streak camera design the beryllium is replaced by a 0.25 micron thick carbon or a 0.1 micron thick Paralene-N $\left(\mathrm{C}_{8} \mathrm{H}_{8}\right)$ film ${ }^{20-22}$ as the x-ray window/photocathode substrate. These films efficiently transmit $x$-rays down to $100 \mathrm{eV}$ (with a gap just above the carbon-K absorption edge). The transmission properties of the $\mathrm{Be}, \mathrm{C}$, and Paralene-N windows are compared in figure 2-1. 


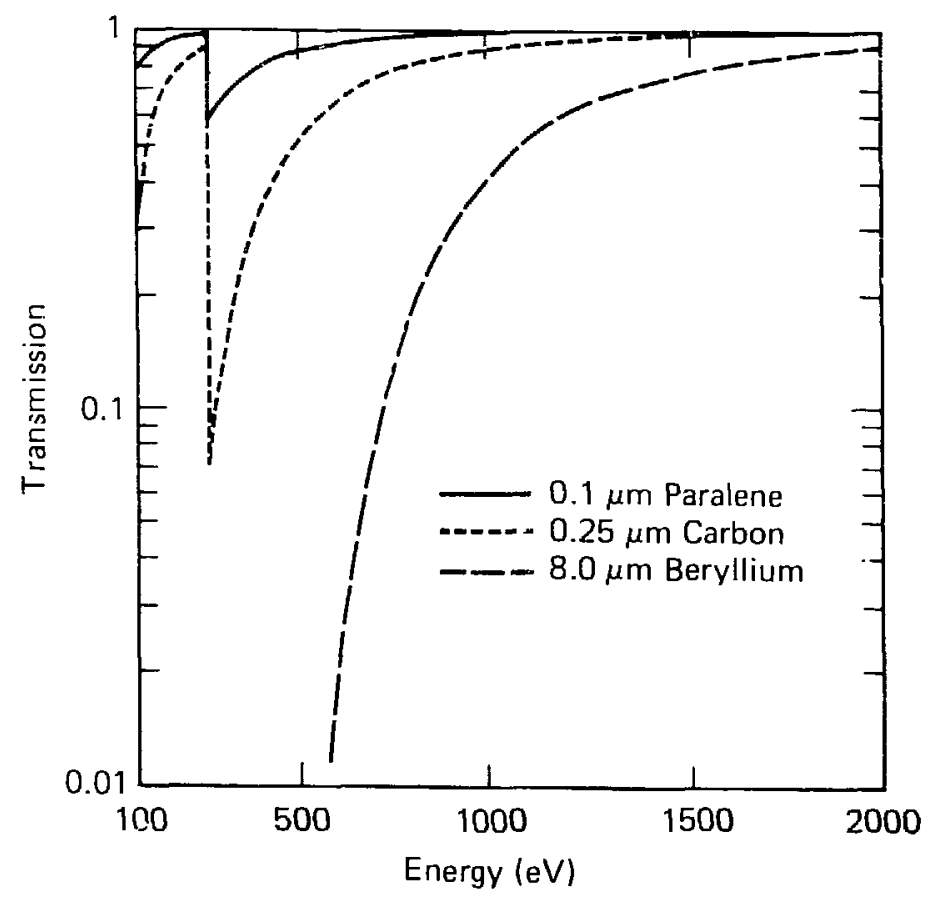

Fig. 2-1. X-ray transmission properties of x-ray streak-camera photocathode substrates. Rolled, 8.0- $4 \mathrm{~m}$ thick beryllium foils are useful for sensitivity above $1 \mathrm{keV}$. Carbon and Paralene-N ( $\left.\mathrm{C}_{8} \mathrm{H}_{8}\right)$ foils $0.25-\mu m$ and $0.1-\mu m$ thick, respectively, provide efficient $x$-ray transmission down to $100 \mathrm{eV}$. The carbon foils are strong but brittle. Paralene-N does not fragment when broken but is less conductive than carbon. 


\section{Soft X-Ray Streak Camera Operation}

The streak camera consists of four major components (figure 2-2): The photocathode, the image converter tube, an image intensifier, and an image recording device. The combined response sharacteristics of these components determine the sensitivity, tempora' and spatial resolution and dynamic range of the complete instrument.

The photon detector. A photocathode converts photon flux into electron smission. Efficiency of the photocathode in terms of emitted elections per incident photon depends on the photoionization efficiency of the material and the secondary electron escape depth. 23,24

A 1 x-ray photon incident on the photocathode is absorbed by or scattered off of an atom, in the process ejecting $a$ high-energy photoelectron. This photoelectron scatters from other atoms in the photocathode, losing energy to loosely-bound secondary electrons which are freed in the process. This cascade process continues until the energetic electrons reach the vacuum interface or until they are therma $1-$ ized. If they have sufficient energy to pass over the vacuum potential shelf they are emitted into the image converter tube and are captured by an extraction field.

The thickness of material traversed by the secondary electron cascade, before the average secondary-electron energy is reduced below the vacuum potential, is the electron escape depth. Clearly, for photoabsorption mean-free-paths greater than the escape defth, as is the case for $x$-ray energies greater than $100 \mathrm{eV}$, the optimum photocathode thickness is the escape depth. 


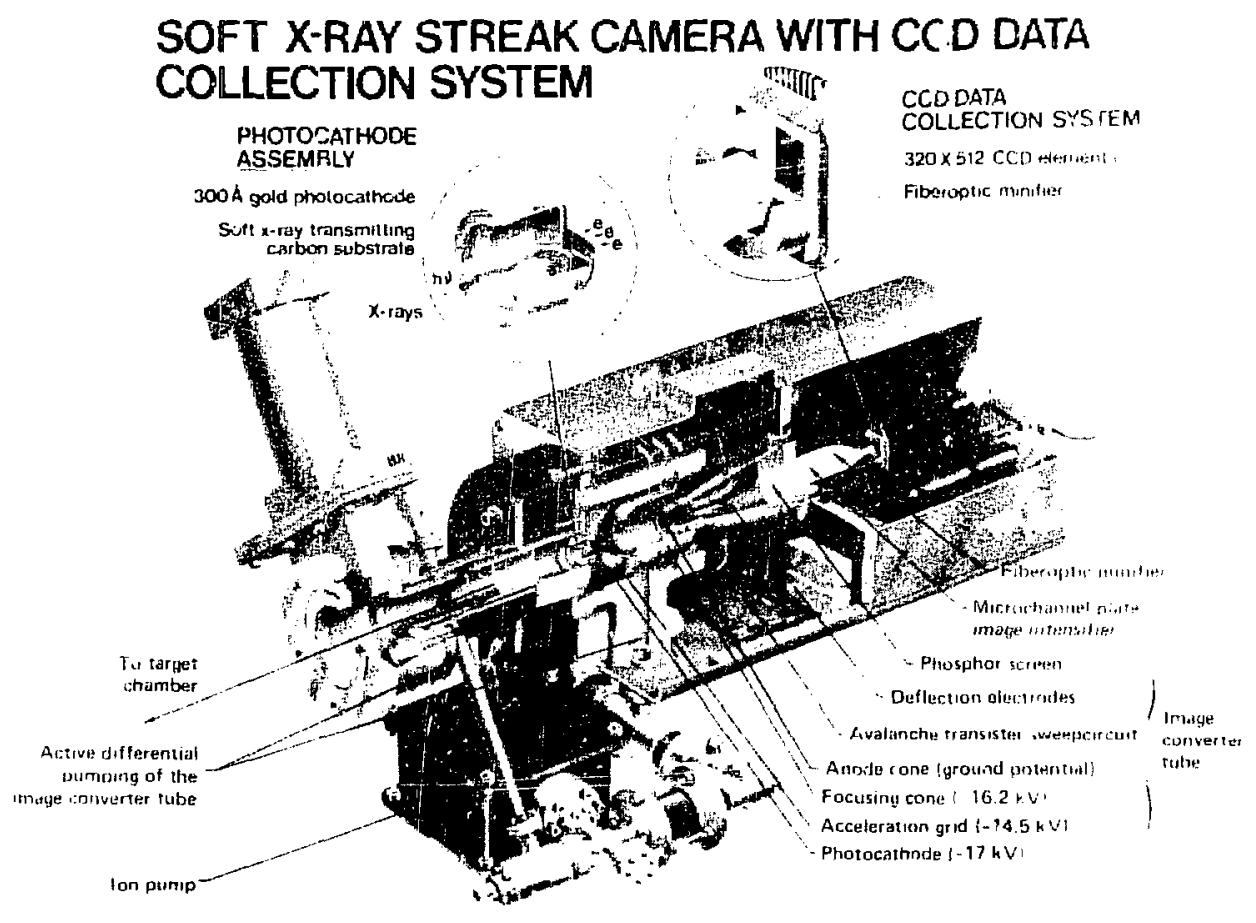

Fig. 2-2. A culaway view of the soft $x$-ray streak camera showing the transmission photocathode and substrate configuration, details of the RCA C73435^ image converter tuhe, a microchannelplate image intensifier and a CCD active data-readout system. Also shown is the active differential vacuum pumping of the ICT and camera front end. 
The electron-scattering cross section, or equivalently the electron mean-free-fath, depends upon the availability of energy states which may be assumed by the electron when it is scattered. For low-energy secondary electrons, a significant band of energy states is forbidden in an insulator which would be available in a conductor thus reducing the scattering cross section for insulators. At a given vacuum potential, an insulator should then have a larger escape depth than a conductor.

If the photon absorption efficiency of an insulator. with a thickness of one escape depth, is greater than the absorption efficiency of a conducting film with a thickness corresponding to its smaller escape depth, the overall electrons-per-incident-photon efficiency of the insulator will be higher. This characteristic of insulating photocathodes has been verified by Henke et al. 24,25

Both conducting gold and insulating cesium iodide have been used as photocathodes in soft $x$-ray streak cameras. Comparison studjes of the temporal response and thickness dependence of the reponse of the two materials ${ }^{26}$ is presented in Chapter 4.

Electron imaging and streaking. The image converter tube (ICT) manipulates the electron emission current from the photocachode to produce a time-resolved streaked image of the incident signal. A rajiograph of the RCA 非73435A image converter tube used in this instrument is shown in figure 2-3. The space current from the photocathode is rapidly accelerated by the $6-\mathrm{kv} / \mathrm{cm}$ extraction field between the cathode and grid. An electrostatic focusing field then images the electron current onto a P-11 aluminum-coated phosphor screen. Between the focusing optics and the phosphor screen, the electron stream passes between 


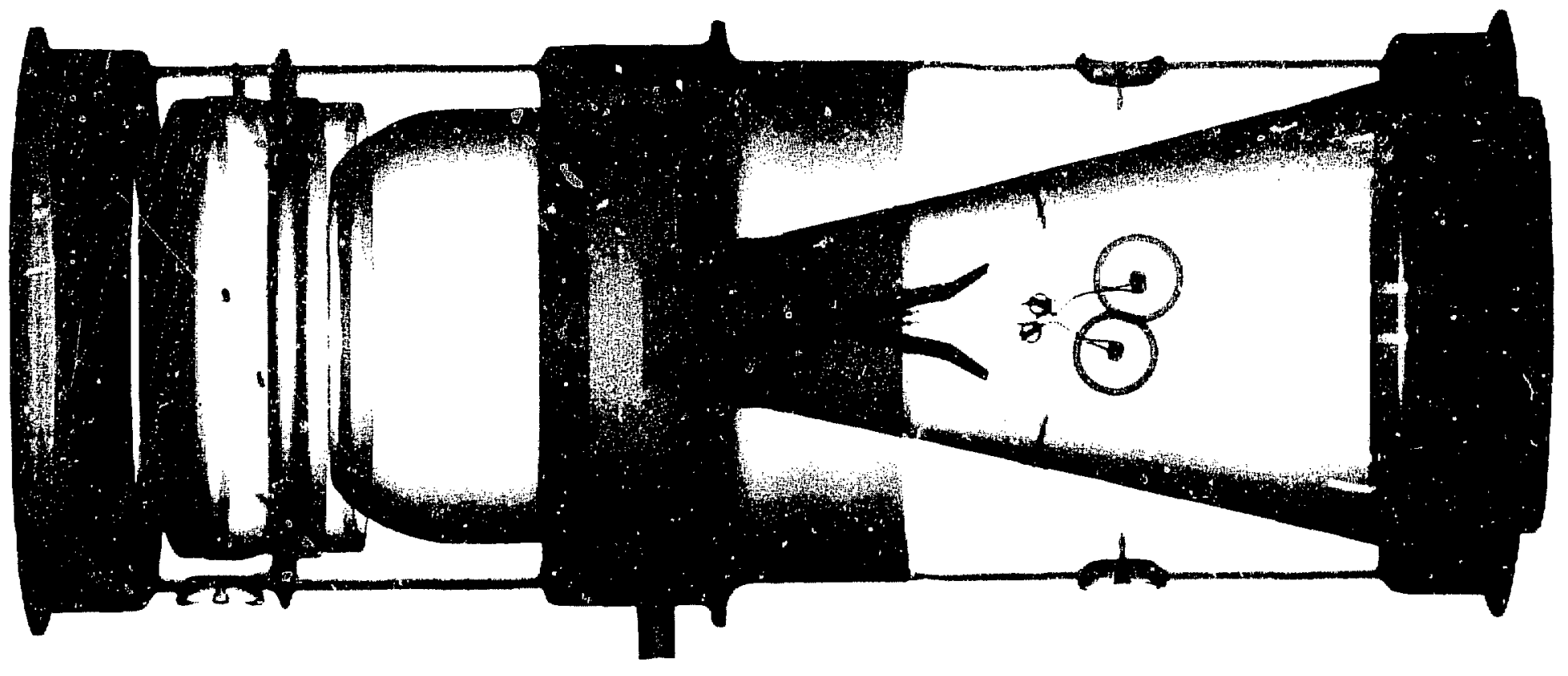

Fig. 2-3. A 10-keV radiograph of the RCA \#C73435A (Blank) image converter tube. 
two deflection electrodes. A ramped voltage between the deflection electrodes is synchronously triggered by a laser pulse to match the ärival time of the $x$-ray signal. The resulting rampeci field deflects the photocathode image across the phosphor screen linearly with time producing a visible streaked imase of the incident x-ray flux with very-high temporal resolution. At LJNL, we have used a 100-um wide by 1-cm long rectangular photocathode to optinize the temporal resolution of the resulting signal. Lerche and Phillips ${ }^{27}$ have recently shown that wider photocathodes may be used for increased detection sensicivity by varying the axially asymetric ICT focus to project a wide emission region onto a narrow image. This imaging is possible due to the perturbation of the axially symmetric focusing fields in the ICT by ihe transverse extraction-grid structure used in this design.

The ICT requires very low operating pressures to avoid electrical breakdown in tigh-field regions and the background noise accompanying such a discharge. In applications where a vacuum-tight beryllium window serves as the photocathode substrate, the ICT is baked out under vacuum and sealed.

The very thin carbon foil or Paralene-N film substrate materials used in the soft $x$-ray streak cameras are very fragile and will not withstand a large pressure differential. Consequently, the ICT is actively pumped with provision to differentially pum across the thin-foil photocathode substrate. The active differentialpumping design may be seen in figure 2-2. An ion pump maintains vacuum through a pumping port in the side of the ICT and may pump on the region between the photocathode and the gate valve as well. 
Prudence dictates that the fragile substrates be replaceable. A demountable photocathode fixture is provided for the soft $x$-ray version - i the streak camera. This feature has proven useful for convenient testing of experimental photocathodes ${ }^{26}$ as well as for a replacement of numerous casualties of "vacuum accidents".

Signal amplification. A mieru-channel-plate image intensifier amplifies the faint streaked image on the phospior screen to recordable levels. The P-11 phosphor screen of the ICT is fiber optically coupled to the 40-mm S-20 photocathode of the intensifier. The electron emission from this photocathode is electrically accelerated along open channels etched through the centers of a two dimensional array of microscopic lead glass fibers as shown in figure 2-4. Cascades of eiectrons are produced by collisions along the walls of the channels and are directed to a P-20 phosphor screen where the amplified data streak is produced with good spatial fidelity. Spatial resolution values of 20 $1 \mathrm{p} / \mathrm{mm}$ and luminous gains of $23 \times 10^{3}$ to $2 \times 10^{4}$ are typical characteristics of the ITT micro-channel plate image intensifiers. ${ }^{28}$

Data recording. The amplified, streaked image is recorded for digital analysis by one of two techniques. The image may be contact recorded on photographic film, which is individually calibrated with an exposure stepwedge for film density-to-data-intensity conversion. 29 Typical images and stepwedges on 4"x5" RXP film are shown in the Appendix, where data reduction and analysis techniques are detailed. The image and the stepwedge are reduced to arrays of density values using a computer controlled microdensitometer. The advantage of the film 


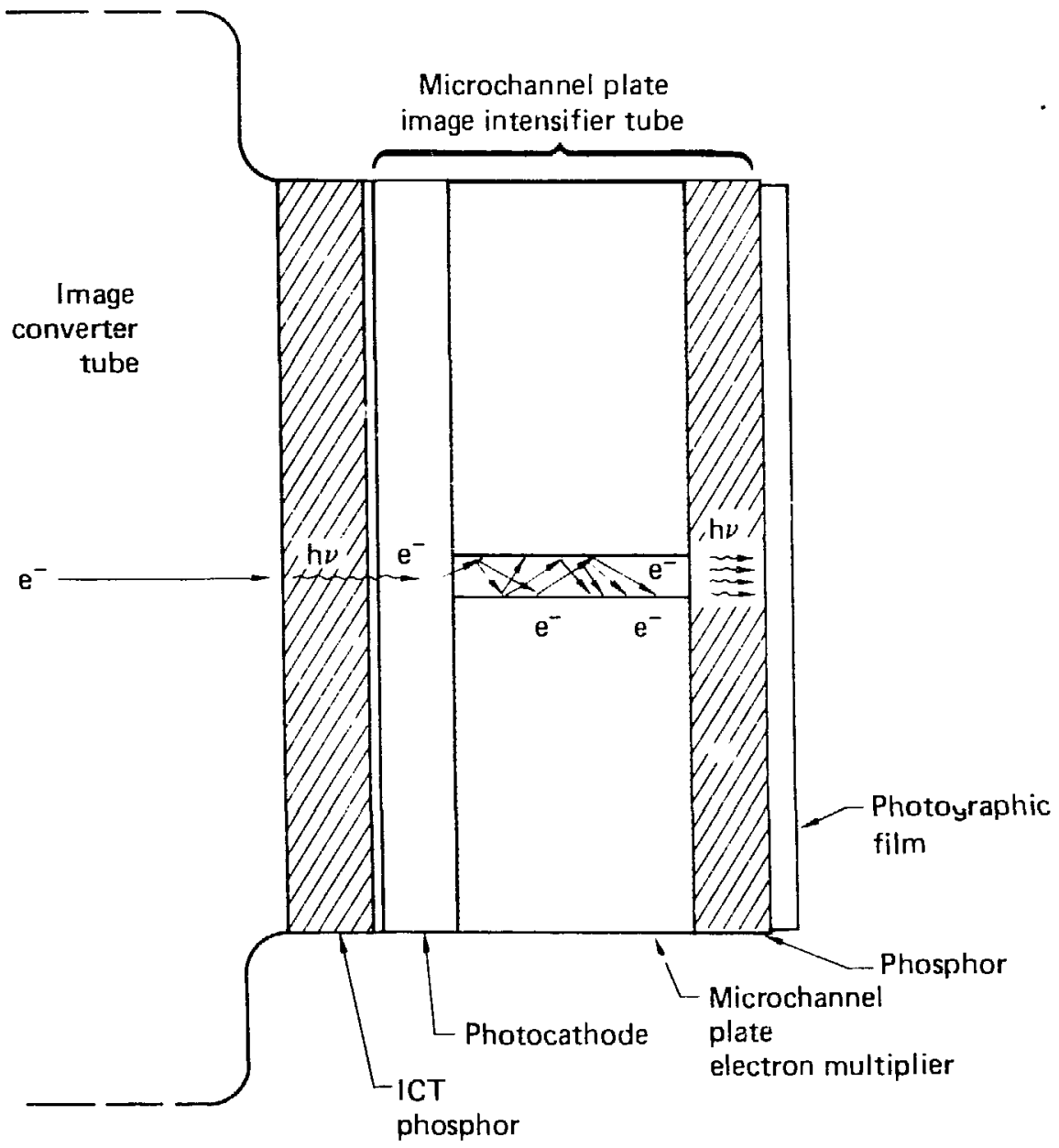

Fig. 2-4. Microchannel-plate image-intensifier design schematic. Photons eject electrons from thie microchannel-plate photocathode. These electrons are accelerated through very narrow lead-glass channels and produce cascades of electrons when they impact the high electron-density walls of the channels. This amplified electron signal strikes a fluorescent screen and produces an image of photographable intensity. 
recording technique is the large dynamic range available with careEully calibrated photographic film. The disadvantage is that film processing and digitization procedures are time consuming and that operator error is inherent in manual processing of film.

A second te:hnique of automatic digital data retrieval as shown in Eigure 2-2 is also used. An array of CCD solid-state detectors ${ }^{30}$ are Eiber optically relayed with the image-intensifier phosphor. A comput:r reads out and stores the detected signal in each pixel oi the array. The dynamic range of state-of-the-art CCD systems is $10^{2}$. This is somewhat less than is available with film. Environments with high $x-r a y$ fluxes or large electromagnetic pulses require additional shielding of the CCD data collection hardware. ${ }^{31}$

\section{System Resolution}

The temporal detail resolvable by the streak camera is limited by the temporal and spatial blurring inherent in the individual signal transfer functions of the camera. If the incident signal were Gaussian in time and the individual resolution contributions were also Gaussian, the total system resolution could be calculated as the quadratic sum 5 of the resolutions of the various contributions:

$$
\tau_{\text {tot }}=\left(\sum_{i} \tau_{i}^{2}\right)^{1 / 2},
$$

where $\tau_{i}$ are the contributions to the temporal resolution limit from the various camera signal transter functions. Although the components of this resolution system will not likely be statistically independent 
Gaussians, this method of analysis can be used to estimate the limits of the total system resolution. 5

Temporal blurring results from transit time dispersion of the electron signal from the photocathode as it traverses the ICT to the optical imaging phosphor. This spread in eransit times depends on the initial distribution of electron velocities and on the field configuration in the tube.

The transit-time dispersion caused by an jaitial electron-energy spread, $\Delta \varepsilon(\mathrm{eV})$ FWHM, accelerated through a region c.f Field strength $E(V / c m)$, can be obtained by a straightforward trajectory calculation to be $:^{7}$

$$
\Delta \tau_{\varepsilon}=3.4 \times 10^{8} \frac{(\Delta \varepsilon)}{E}^{1 / 2} \quad \text { (psec) }
$$

By approximating the field configuration along the axis of the tube as stepped regions of constant field strength, Jaanimagi ${ }^{32}$ has found the total transit-time dispersion to be:

$$
\Delta \tau_{\varepsilon}=7.1(\Delta \varepsilon)^{1 / 2}+0.60 \Delta \varepsilon \quad(\mathrm{psec})
$$

A correction term, $\Delta \tau_{c} \leq 1.5 \Delta \varepsilon$ (psec) has been proposed by Jaanimagi for dispersion induced in high-charge-density crossover regions of the ICT. Thus the approximate dynamic dispersion would be: ${ }^{32}$

$$
\Delta \tau_{\text {disp }} \simeq 7.1(\Delta \varepsilon)^{1 / 2}+2.1 \Delta \varepsilon \quad(\mathrm{psec})
$$

This method of analysis has been observed to closely match trajectory calculations for more detailed field distributions. 
recording technique is the large dynamic range available with carefully calibrated photographic film. The disadvantage is that film processing and digitization procedures are time consuming and that operator error is inherent in manual processing of film.

A second technique of automatic digital data retrieval as shown in figure 2-2 is also used. An array of CCD solid-state detectors 30 are fiber opticaliy relayed with the image-intensifier phosphor. A computer reads out and stores the detected signal in each pixel of the array. The dynamic range of state-of-the-art CCD systems is $10^{2}$. This is somewhat less than is available with film. Environments with high $x$-ray fluxes or large electromagnetic pulses require additional shielding of the CCD data collection hardware. 31

\section{System Resolution}

The temporal detail resolvable by the streak camera is limited by the temporal and spatial blurring inherent in the individual signal trinsfer functions of the camera. If the incident signal were Gaussian in time and the individual resolution contributions were also Gaussian, the total system resolution could be calculated as the quadratic sum ${ }^{5}$ of the resolutions of the various contributions:

$$
\tau_{\text {tot }}=\left(\sum_{i} \tau_{i}^{2}\right)^{1 / 2},
$$

where $\tau_{i}$ are the contributions to the temporal resolution limit from the various camera signal transfer functions. Although the components of this resolution system will not likely be statistically independent 
Gaussians, this method of analysis can be used to estimate the limits of the total system resolution. 5

Temporal blurring results from transit time dispeision of the electron signal from the photocathode as it traverses the ICT to the optical imaging phosphor. This spread in transit times depends on tine initial distribution of electron velocities and on the field configuration in the tube.

The transit-time dispersion caused by an initial electron-energy spread, $\Delta E(e V)$ FWHM, accelerated through a region of field strength $E(V / c m)$, can be obtained by a straightforward trajectory calculation to be : ${ }^{7}$

$$
\Delta \tau_{E}=3.4 \times 10^{8} \frac{(\Delta \varepsilon)^{1 / 2}}{E} \quad(\mathrm{psec})
$$

By approximating the field configuration along the axis of the tube as stepped regions of constant field strength, Jaanimagi ${ }^{32}$ has found the total transit-time dispersion to be:

$$
\Delta \tau_{\varepsilon}=7.1(\Delta \varepsilon)^{1 / 2}+0.60 \Delta \varepsilon \quad(\text { psec })
$$

A correction term, $\Delta \tau_{c} \leq 1.5 \Delta \varepsilon$ (psec) has been proposed by Jaanimagi for dispersion induced in high-charge-density crossover regions of the ICT. Thus the approximate dynamic dispersion would be: ${ }^{32}$

$$
\Delta \tau_{\mathrm{disp}} \simeq 7.1(\Delta \varepsilon)^{1 / 2}+2.1 \Delta \varepsilon \quad(\mathrm{psec})
$$

This method of analysis has been observed to closely match trajectory calculations for more detailed field distributions. 
Henke et al..$^{25}$ have measured the secondary-electron-emission energy distribution of a variety of photocathode materials. They have reported energy spreads for Au and CsI of $3.9 \mathrm{eV}$ and $1.6 \mathrm{eV}$ respectively. Using the newly proposed dynamic dispersion of Jaanimagi $\Delta \tau_{\text {disp }}$ for an Au photocathode is then 222 psec and for a CsI photocathode is 212 psec, which are somewhat larger values than previously used, but not inconsistent with presently available data.

The temporal resolution of the system is also constrained by dc image resolution at the camera output in the sweep direction. This minimum image size in the time dimension of the LLNL/RCA streak camera system has been observed by Lerche and Phillips ${ }^{27}$,o be $175 \mu \mathrm{m}$. A range of photocathode slit widths were focused to this minimum size without accompanying distortions in the transverse resolut...

The time required to sweep the image through its spatial extent is the static resolution contribution to the temporal resolution:

$$
\Delta \tau_{s}=v d \quad(p s e c)
$$

where $d(u m)$ is the static image size in the time dimension and $V(\mathrm{psec} / \mathrm{mm})$ is the inverse speed of the sweep.

The fastest sweep circuit available for this instrument produces a 34-psec/mm inverse sweep speed with a corresponding static contribution to the temporal resolution of $6.0 \mathrm{psec}$.

The limit of resolution with a $34 \mathrm{psec} / \mathrm{mm}$ sweep circuit and a CsI photocathode can be approximated by:

$$
\Delta \tau_{\text {tot }} \simeq\left[\Delta \tau_{\text {disp, }}^{2} \text { ss }+\Delta \tau_{s}^{2}\right] 1 / 2
$$




$$
\Delta \tau_{\text {tot }} \simeq\left[(12 \mathrm{psec})^{2}+(6.0 \mathrm{psec})^{2}\right]^{1 / 2}=13.7 \mathrm{psec}
$$

A direct measurement ${ }^{33}$ of the time resolution limit of the system would be preferable to indirect calculations. A very short pulse, 21-psec, x-ray source is not currently available to us, but measurements of $30-p s e c$-ray pulses with a $34 \mathrm{psec} / \mathrm{mm}$ sweep card are not inconsistent with a value of $\Delta \tau_{\text {tot }}$ of approximately $15 \mathrm{psec}$.

The temporal measurements presented in this Dissertation purposely used sweep rates three times slower than maximum to allow temporal histories as long as several nanoseconds to be recorded. In these measurements state of the art temporal resolution is not required. Two soft $x$-ray streak cameras were used to take these data: SX-1 (soft $x$-ray streak camera 非) with a gold photocathode and an inverse sweep rate of $134 \pm 7 \mathrm{psec} / \mathrm{mm}$ and SX-6 with a CsI photocathode and an inverse sweep rate of $116 \pm 6 \mathrm{psec} / \mathrm{mm}$. $\tau_{\mathrm{s}, \mathrm{SX} 1}$ is $23.5 \mathrm{psec}$ and $\tau_{\mathrm{s}, \mathrm{SX} 6}$ is $20 \mathrm{psec}$. Thus the temporal resolution of $\mathrm{sX}-1$ measurements is:

$$
\Delta \tau_{\mathrm{SX} 1} \simeq\left(\Delta \tau_{\text {disp,Au }}^{2}+\Delta \tau_{\mathrm{s}, \mathrm{sX} 1}^{2}\right)^{1 / 2}
$$

and

$$
\Delta \tau_{S \times 1} \simeq 32 \mathrm{psec}
$$

For $\mathrm{SX}-6$ we have:

$$
\Delta \tau_{\mathrm{SX} 6} \simeq\left(\Delta \tau_{\mathrm{disP}, \mathrm{CsI}}^{2}+\Delta \tau_{\mathrm{s}, \mathrm{SX6}}^{2}\right)^{1 / 2}
$$

and

$$
\Delta \tau_{\text {SX6 }} \simeq 24 \mathrm{psec}
$$

The effect of a non-zero resolution limit on the measured signal can be roughly estimated with the Gaussian quadrature approximation used 
above. For example, a 100-psec signal detected with a 32-psec resolution system will be broadened by $ح 5$ percent. X-ray-emission temporal deraii on a scale relevant to laser fusion experiments ( 2100 psec) are thus well resolved with this conservative system resolution. 


\section{$\underline{\text { References }}$}

1. C. F. McConaghy and L. W. Coleman, Appl. Phys. Lett. 25, 268 (1974).

2. D. T. Attwood, IEEE J. Quantum Electron, QE-14, 909 (1978).

3. G. L. Stradling, D. T. Attwood, J. W. Houghton, E. L. Pierce, and D. P. Gaines, Bull. Am. Phys. Soc. 23, 880 (1978).

4. G. L. Stradling, M. S. Thesis, Brigham Young University, 1981, aiso Lawrence Livermore National Laboratory, UCRL-52568.

5. E. K. Zaroiskii and S. D. Fanchenko, Dok, Akad. Nauk. SSSR ISov. Phys. Dokl.]. Vol. 10, 66l (1955); Dok. Akad. Nauk. SSSR [Sov. Phys. Dok1.1, Vol. 108, 218 (1955); Appl. Opt. 古, 1155 (1965).

6. V. V. Korobkin and ir. Ya. Schelev, Proceedings of the 8 th International Congress on High-Speed photography, Stockholm (Wiley, New York, 1968), p. 36 .

7. V. V. Korobkin, A. A. Malyutin, and M. Ya. Schelev, J. Photogr. Sci., 17, 179 (1969).

8. D. J. Bradley, B. Liddy, and W. E. Sleat, Opt. Commun. 2 , 391 (1971).

9. D. J. Bradley, B. Liddy, W. Sibbett, and W. E. Sleat, App? Phys. Lett. 20, 219 (1972).

10. M. Ya. Schelev, M. C. Richardson, and A. J. Alcock, Appl. Phys. Lett. 18,354 (1971).

11. M. Ya. Schelev, M. C. Richardson, and A. J. Alcock, Reiv. Sci. Instrum. 43, 1819 (1972).

12. M. M. Buslov, V. V. Korobkin, A. M. Prokhorov, B. M. Stepanov, and M. Ya Schelev, Proceedings of the loth International Congress on High-Speed Photography, edited by E. Laviron (Association Nationale de la Recherche Technique, Paris, France, 1972), p. 122.

13. N. G. Basov, et al., presented at the Conference on Nonlinear Optics, Minsk, USSR, 1972 (unpublished).

14. S. W. Thomas and L. W. Coleman, Appl. Phys. Lett. 20, 83 (1972).

15. S. i. Thomas, G. R. Tripp, and L. W. Coleman, Proceedings of the 10th International Congress on High-Speed Photography, edited by E. Laviron (Association Nationale de la Recherche Technique, Paris, France, 1972) p. 127 .

16. L. W. Coleman and S. W. Thomas, IEEE J. Quant. Elec. QE-9, 713 (1973). 
17. L. W. Coleman, presented at the Society of Photo-optics Instruments and E.ıgineering Meeting, San Diego, Cal., 1973 (unpublished).

18. R. Kalibjain, in Proceedings of the 13th International Congress on High Speed Photography and Photonics, edited by Shin-Ichi Hyodo (Japan Society of Precision Engineering, Tokyo, Japan, 1979), p. 452.

19. S. W. Thomas and G. E. Phillips, In Proceedings of the 13th International Congress on High Speed Photography and Photonics, edited by Shin-Ichi Hyodo (Japan Society of Precision Engineering, Tokyo, Japan, 1979), p. 471; S. N. Thomas, Ibid. p. 838.

20.W.F. Gorham, J. Poly. Sci. 4, 3027 (1966).

21. M. A. Spivack, Rev. Sci. Instrum. 41, 1614 (1970).

22. Paralene-N used in the soft $x$-ray streak cameras is obtained from Lebow Co., Goleta, Ca., who manufactures it under license from Union Carbide.

23. B. L. Henke, J. A. Smith, D. T. Attwood, Appl. Phys. Lett. 29, 539 (1976).

24. B. L. Henke, J. P. Knauer, and K. Permeratne, J. Appl. Phys. 52, $1509(1981)$.

25. B. L. Henke, J. Liesgang, and S. D. Smith, Phys. Rev. Bl9, 3004 (1979).

26. G. L. Stradling, H. Medecki, R. L. Kauffman, D. T. Attwood, and B. L. Henke, Appl. Phys. Lett. 37, 782 (1980).

27. R. A. Lerche and G. E. Phillips, Bull. Am. Phys. Sor., 26, 910 (1981); also Lawrence Tivermore Nationa1 Laboratory, UCRL-86405.

28. ITT Electro-Optical Products Division, Data Sheets, Generation II Proximity Focused Channel Intensifier Tubes, 2-72; F-4117, 7-77.

29. C. H. Dittmore established and maintains the excellent sensiometric facilities available at LLN''s Tech Photo group.

30. J. Cheng, G. R. Tripp, and L. W. Coleman, presented at the International Conference on the Application of CCD Devices, San Diego, Cal., 1978.

31. J. T. Noonan, H. Medecki, and R. L. Kauffman, private communication (1980).

32. P. A. Jaanimagi, Ph.D. Thesis, University of Waterloo, Waterloo, Ontario, Canada, 1981.

33. D. J. Bradley, A. G. Roddio, W. Sibbett, M. H. Key, M. J. Lamb, C. L. S. Lewis, and P. Sachsenmaier, Opt. Commun. 15, 231 (1975). 
Chapter 3

\author{
SPECTRAL DISCRIMINATION TECHNIQUES: \\ ABSORPTION-EDGE FILTERING, TOTAL EXTERNAL REFLECTION, \\ AND BRAGG REFLECTION WITH METAL MULTILAYERS
}

\title{
Introduction
}

$X-r a y$ emission from a laser-heated plasma carries information about energy balance and light-to-x-ray conversion efficiency in the experiment as well as information about the temperature, electron-density geometry and ionization states of the emission region. However, the $x$-ray flux must be measured with meaningful resolution of spectral, temporal, and spatial parameters. Such detailed x-ray measurements, combined with other ccmplimentary measurements provide access to important physical processes in the experiment.

The degree of spectral resolution required in a diagnostic instrunent depends on the t/pe of measurement to be made. Knowledge of the light-to-x-ray conversion efficiency and the general shape of the spectral envelope may be obtained with broad-band spectral channels covering the more intense regions of the spectrum. The history of line emission from the plasma requires narrower channels located at appropriate spectral energies. Line shapes and detailed spectral features require continuous high-resolution coverage over the region of interest.

Manipulation of $x$ rays in the $100-e V$ to $1000-e V$ spectral region is made challenging by the characteristically strong absorption of subkilovolt photons in all matter and by the difficulty of abtaining periodic structures on the order of $30 \AA-100 \AA$ suitable for the efficient diffraction of $x$-rays at these energies.

This chapter will describe some appropriate spectral discrimination techniques used in time-resolved soft $x$-ray measurements, their advantages and disadvantages. Specifically, techniques applying absorptionedge filters, total-external-reflection mirrors and metal-multilayer* $x-r a y$ interference mirrors will be discussed.

\footnotetext{
* The term metal multilayer is commonly used to distinguish the sputtered multilayers from the stearate type of synthetic multilayers. To this point, metals have been used exclusively as the scattering layers in multilayers. Non-metals may serve this scattering function in future efforts.
} 


\section{Absorption-Edge Filtering}

Rough spectral discrimination with thin absorption foils can be obtained using the energy-dependent mass-absorption features characteristic of atomic-resonance thresholds. Exponentiaily decreasing transmission efficiency through a foil with decreasing photon energy may be used to filter out the low-energy component of a plasma emission spectrum. The sharp drop in transmission efficiency at an absorption edge followed by a gradual rise of transmission with increasing photon energy will also discriminate against photons in the energy band immediately above the absorption edge. These transmission characteristics are apparent in the carbon-filcer function in figure 2-1.

This filtering feature can be used most effectively if the $x$-ray spectrum falls rapidly with increasing photon energy and has negligible contribution in the rising transmission region above the absorption edge of the filter. In such cases, spectral resolution $E / \Delta E$ of $<4$ are obtainable. Without a falling spectrum, contributions to the transmission lobe below the absorption edge and to the transmission region above the absorption edge significantly degrade the channel resolution. Even when the spectrum is not falling, however, some spectral detail may be obtained by self-consistently unfolding the response of a number of different filtered channels. ${ }^{1,2}$ This analysis requires careful calibration of the individual spectral response-functions of the various channels.

While absorption filtering can obtain only broad spectral discrimination in a limited number of naturally occuring spectral windows, the 
large throughrit obtainable with this technique enhances measurement statistics.

\section{Total External X-Ray Reflection}

The resolution of an energy channel obtained with an absorption-edge filter may be increased using an $x$-ray mirror to cut off transmitted signal above the absorption edge. ${ }^{3}$ The effectiveness of this technique is illustrated in figure 3-1. Resolution values E/ $\mathrm{E}$ of 3-5 may be obtained with this reflection technique, even in the presence of a non-decaying emission spectrum. X-ray reflecting mirrors have been used here for the first time with $x$-ray streak cameras for highenergy signal suppression.

Total external reflection of x-rays from a polished mirror occurs at grazing angles for photon energies up to a critical energy $E_{c}$. Portions of the incident signal with photon energies above the critical energy are strongly absorbed and reflection is dramatically attenuated.

This phenomena is the result of the refractive index, $n$, dipping below unity in the $x$-ray region of the spectrum. ${ }^{3}$

\section{Theory}

The low-pass filter, high-energy cutoff character of total external reflection in the $x$-ray regime is due to the energy dependent index of refraction $n(E)$ which increases toward unity with increasing photon energy .

The dielectric function $\varepsilon=n^{2}$ may be written $\mathbf{a s}^{4}$ :

$$
E(E)=1-\frac{4 \pi N e^{2} \hbar^{2}}{m_{e}} \sum_{i j} f_{i j}\left(E^{2}-E_{i j}^{2}+i E g_{i j}\right)^{-1}
$$



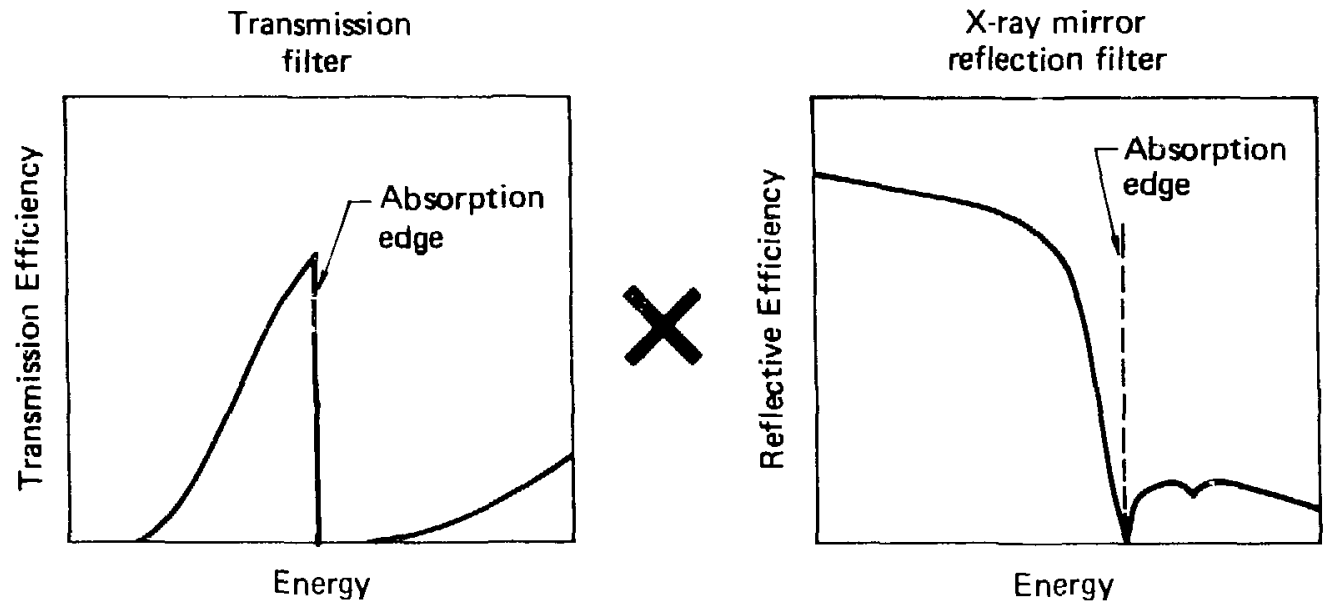

Channel

response

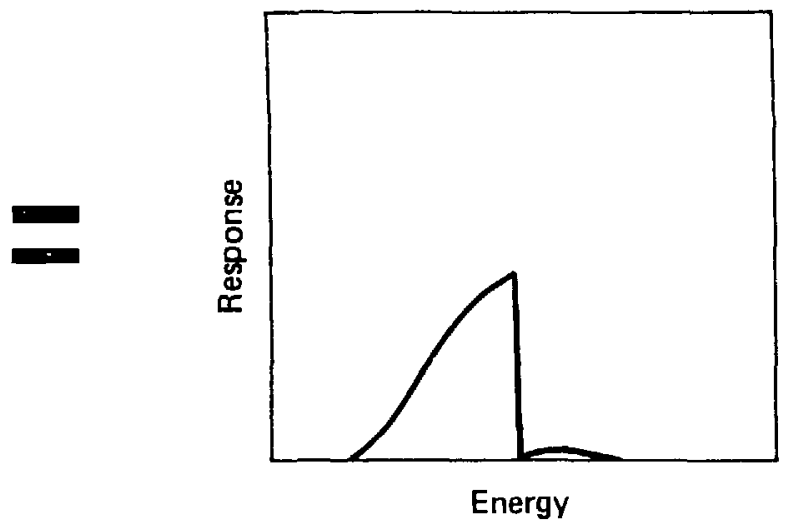

Fig. 3-1. Reflector/filter channel discriminator design. The absorption edge of a transmission filter combined with the high-energy fall-off in reflectivity of an $x$-ray mirror provide a moderately narrow energy channel below the spectral peak. 
where, in this semi-classical discription, $F_{i j}$ is the oscillator strength of the $j^{\text {th }}$ transition (which has the energy $E_{i j}$ ) of the $i^{\text {th }}$ electron in the atom and which has a damping constant $g_{i j}$. The photon energy, electron charge and mass are $E, e$, and $m$ respectively. There are $N$ atoms per unit volume and $Z$ electrons per atom. The oscillator strenth $\mathrm{f}_{\mathrm{ij}}$ satisfies the sum rules,

$$
\begin{aligned}
& \sum_{j} E_{i j}=1 \\
& \sum_{i j} F_{i j}=z .
\end{aligned}
$$

Total external reflection results when the angle of incidence $\theta_{i}$ exceeds the critical angle,

$$
\theta_{c}=\sin ^{-1}[n(E)],
$$

which is a function of the index of refraction. In this case the refracted wave vector is parallel to the vacuum mirror interface and no energy is propagated into the medium. 5 Consequently all of the incident energy is reflected. For a given grazing angle of incidence 'inc, the total reflection condition is met if the index of refraction $n(E)$ is less than the critical value obtained from Snells law:

$$
\mathrm{n}_{\mathrm{c}}(\mathrm{E})=\sin \theta_{\text {inc }}
$$

Thus for photon energies below the critical energy, which corresponds to the critical refractive index, the incident flux is not transmitted through the mirror/vacuum interface but is reflected. At 
photon erergies above the critical energy, $x$-rays are refracted into the mirror and are strongly absorbed. Calculations of the reflectivity ${ }^{6}$ variation with photon energy of carbon at a $5^{\circ}$ grazing angle and of nickel at grazing angles of $3^{\circ}$ and $5^{\circ}$ are plotted in figure 3-2.

For a critical-energy value not near an absorption edge, i.e. $E=$ $E_{i j}$, the steepness of the reflectivity cutoff is strongly affected ${ }^{3}$ by the size of the damping terms $g_{i j}$ in $E(E)$ compared to the photon energy, $E$. Large values of the damping term degrade the sharpness of the cutoff through increased extinction of the evanescent signal at photon energies belcw the critical energy.

A total-external-reflector and an absorption-edge filter may be used in series for enhanced spectral resolution. The high-energy cutoff is enhanced with the critical energy of the reflector positioned slightly below the absorption edge of the filter material. The shape of the resulting channel response is then the filter transmission below the absorption edge. Above the absorption edge, the strong absorption of the filter complements the falling reflectance of the mirror, resulting in negligible contribution to the channel response.

\section{Implementation of Reflector-Filter Spectral Channels}

The first soft $x$-ray streak camera $(S X-1)$ was fielded in 1978 with three different reflector-filter channels. ${ }^{7,8}$ A vitreous carbon mirror was matched at a $5^{\circ}$ grazing angle with a $400-\mu g / \mathrm{cm}^{2}$ carbon absorption filter. Two nickel mirrors at $5^{\circ}$ and $3^{\circ}$ grazing angles were paired with $500-\mu \mathrm{g} / \mathrm{cm}^{2}$ vanadium and $700-\mu \mathrm{g} / \mathrm{cm}^{2}$ iron filter foils respectively. The total response of these channels is illustrated in figure $3-3$. The total system response is calculated by taking the product of the photon-energy 


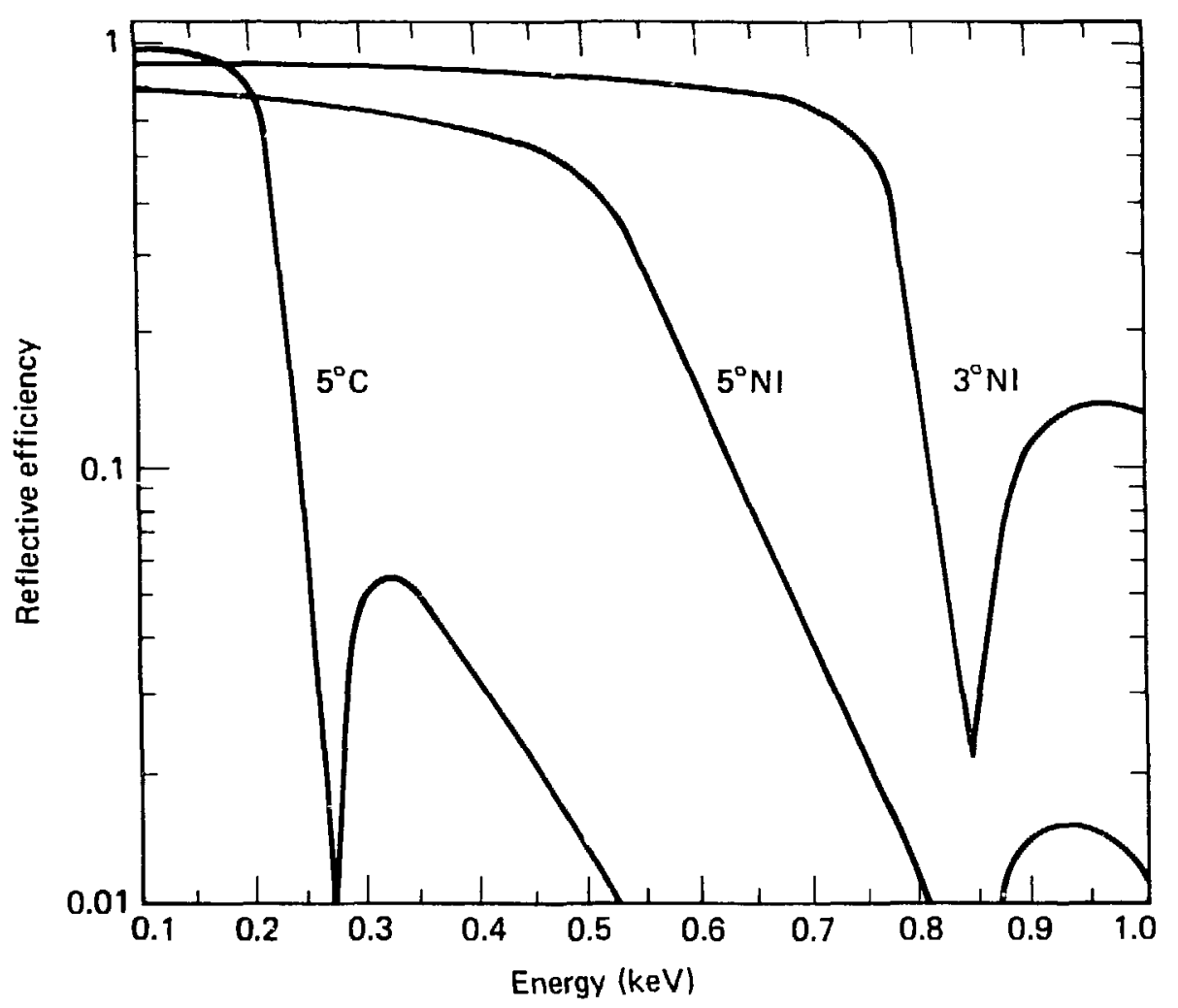

Fig. 3-2. Efficiencies of three selected $x$-ray reflectors: carbon at $5^{\circ}$, nickel at $5^{\circ}$, end nickel at $3^{\circ}$. Reflectivity falls off above the angle-dependent critical energy $E_{c}$, and is strongly affected by resonance absorption structure in the material. 

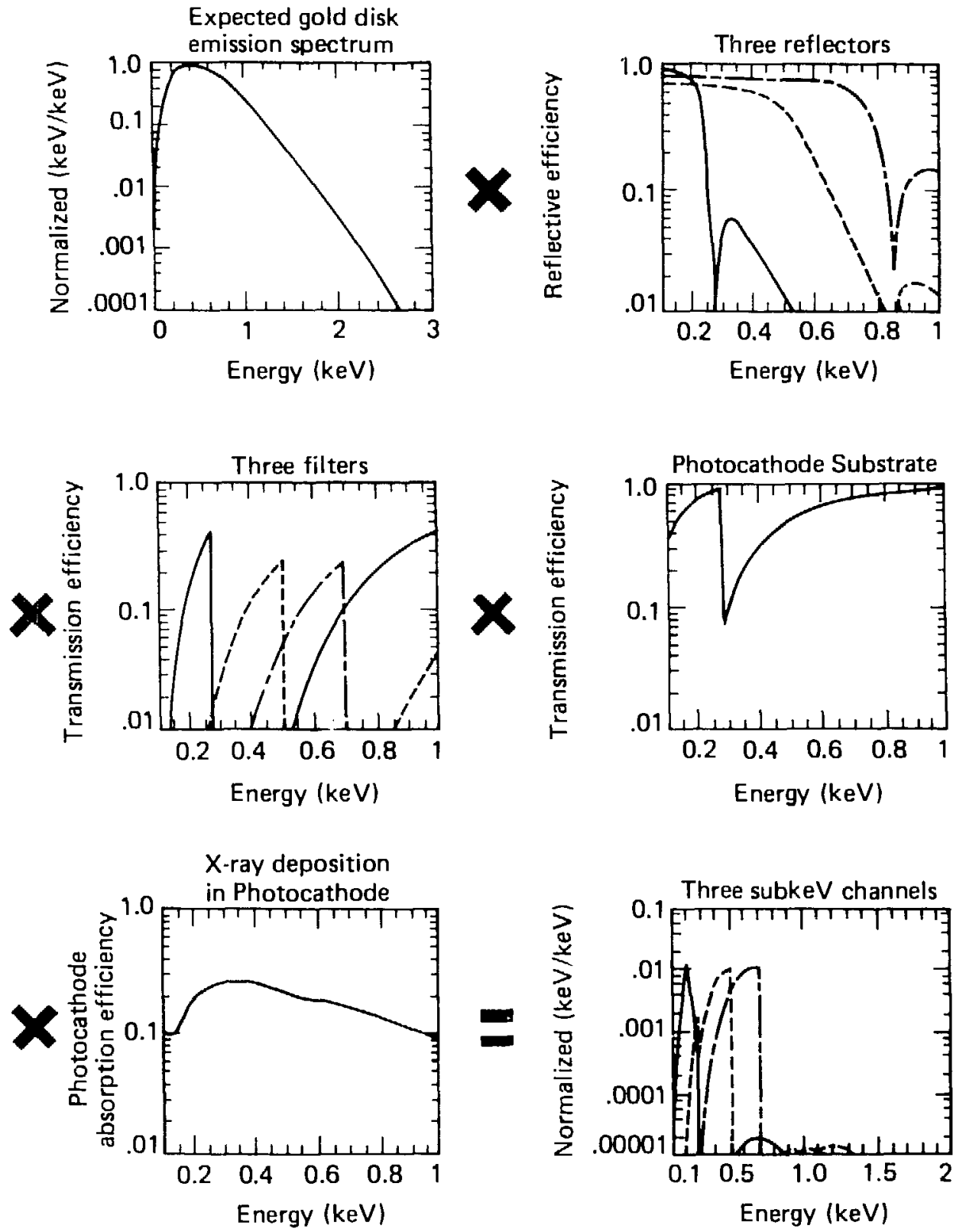

Fig. 3-3. Three-channel reflector/filter design. The three broadband spectral channels are obtained by folding together the source spectrum, the reflective efficiencies of the $x$-ray mirrors, the transmission efficiencies of the absorption filters, the transmission efficiency of the photocathode substrate, and the photocathode conversion efficiency. 
dependent responses of the mirrors, the filter foils, the photocathode and carbon substrate. The absorption-filter thicknesses were selected to give nearly uniform signal levels in the three channels from an incident emission spectrum characteristic of a laser-heated gold disk. Recently, the filter thicknesses on $S x-1$ have been reduced to $300-\mu \mathrm{g} / \mathrm{cm}^{2} \quad$ carbon, ${ }^{9} \quad 220-\mu \mathrm{g} / \mathrm{cm}^{2}$ vanadium, 10 and $310-\mu \mathrm{g} / \mathrm{cm}^{2}$ iron, 10 providing a closer match to the filtering of several XRD detectors in the Dante system. ${ }^{1}$ This change allowed absolute calibration of the SX-1 channels through comparisons with XRD measurements. 11 The spectral responses of these channels are given in figure 3-4. The schematic configuration of this first soft $x$-ray streak camera with reflector/filter spectral discrimination is shown in figure 3-5.

\section{Metal-Multilayer X-Ray Interference Mirrors}

Very respectable spectral resolution and impressive integrated reflectivity coupled with tremendous design flexibility has recently become available in the form of synthetic Bragg reflectors. These devices consist of alternating microscopic layers of materials which have a large contrast in refractive indices at photon energies of interest. These intermediate-resolution, multilayer, $x$-ray interference mirrors have also been used for the first time with $x$-ray streak cameras in the plasma studies reported here.

Both vacuum evaporation 12,13 and sputtering techniques ${ }^{14}$ have been used successfully to fabricate these multilayered structures. Several synonymous names are used in the literature referring to these new synthetic multilayers including: layered synthetic microstructures 


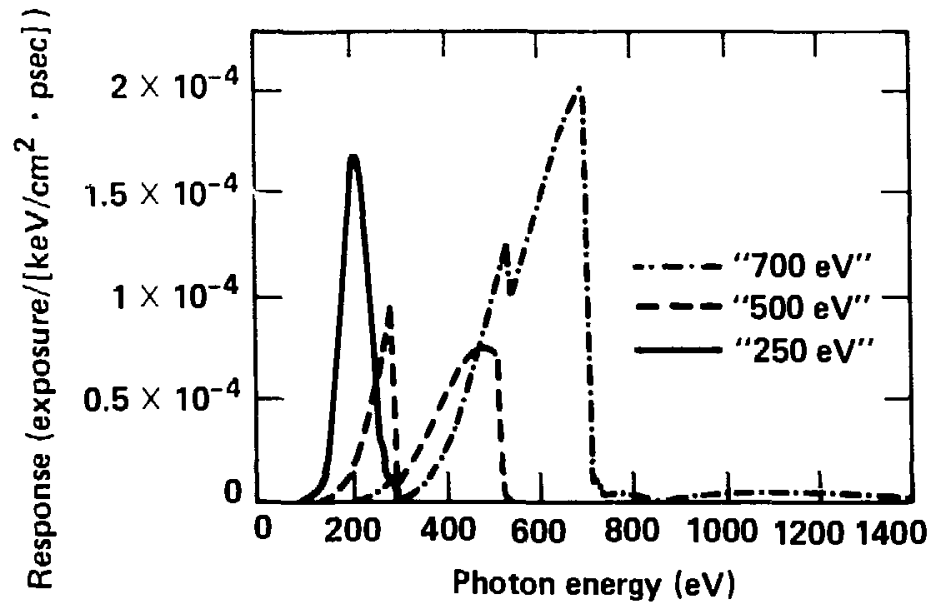

Fig. 3-4. The three reflector/filter spectral channel shapes of $\mathrm{sX}-1$ used for the data presented in this Dissertation. The $2200-e V$ carbon channel consists of a $300-\mu g / \mathrm{cm}^{2}$ vanadium foil and a $5^{\circ}$ nickel reflector. The $\nu 700-\mathrm{eV}$ iron channel uses a $310-\mu \mathrm{g} / \mathrm{cm}^{2}$ iron filter and a $3^{\circ}$ nickel reflector. Included in these spectral window shapes are the $50-\mu g / \mathrm{cm}^{2}$ carbon photocathode substrate and the 300-A thick Au photocathode response. 


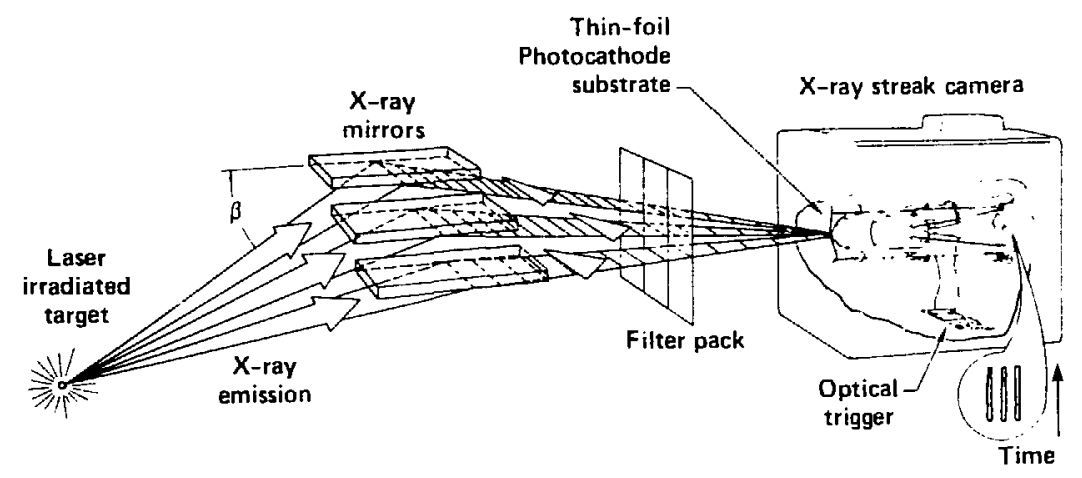

Fig. 3-5. Schematic configuration of the reflector/filter spectral discriminator system on $S X-1$. $X$ rays with energies below $E_{c}$ from the laser-plasma source are reflected from the mirrors and are filtered by the thin-foil absorption-edge filters and the photocathode substrate. 
(LSM), metal multilayers, evaporated/sputtered multilayers (ESM), and multilaver $x$-ray interference mirrors.

\section{Advantages and Characteristics}

These metal multilayer structures diffract $x$-rays in the same way as the smaller d-spacing, natural-crystal, nlane structures or the fixedd-spacing molecular multilayers. ${ }^{15} \mathrm{x}$ rays coherently scatter from the parallel planes of material interfaces. Increased refractive-index contrast enhances the scattering efficiency. At certain reflection angles, which are wavelength dependent, the scattered waves constructively interfere to give Bragg diffraction peaks. Constructive interference as shown in figure 3-6 occurs when the difference in path length $2 d$ $\sin \theta_{i}$, between rays scattered from the two adjacent planes of separation $d$, is equal to an integral number of wavelengths:

$$
\mathrm{m} \lambda=2 \mathrm{~d} \sin \theta_{i}
$$

The amplitude of the diffracted wave as well as the angular divergence possible for the diffracted beam depend upon the number of planes which contribute to the diffracted beam. In the subkev $x$-ray region, absorption properties of available materials limit the number of diffraction planes $\mathrm{N}$ which can appreciably add to the diffracted beam. For this reason, obtainable energy resolution, $E / \Delta E{ }_{N}$, depends strongly on the photon energy and the material components of the multilayer.

High peak reflectivity is always desirable. The energy resolution needed depends upon the specific application. In some instances, where available $x$-zay flux is low or where line structure in the spectrum might distort energy-band measurements of spectral shape, noderately- 


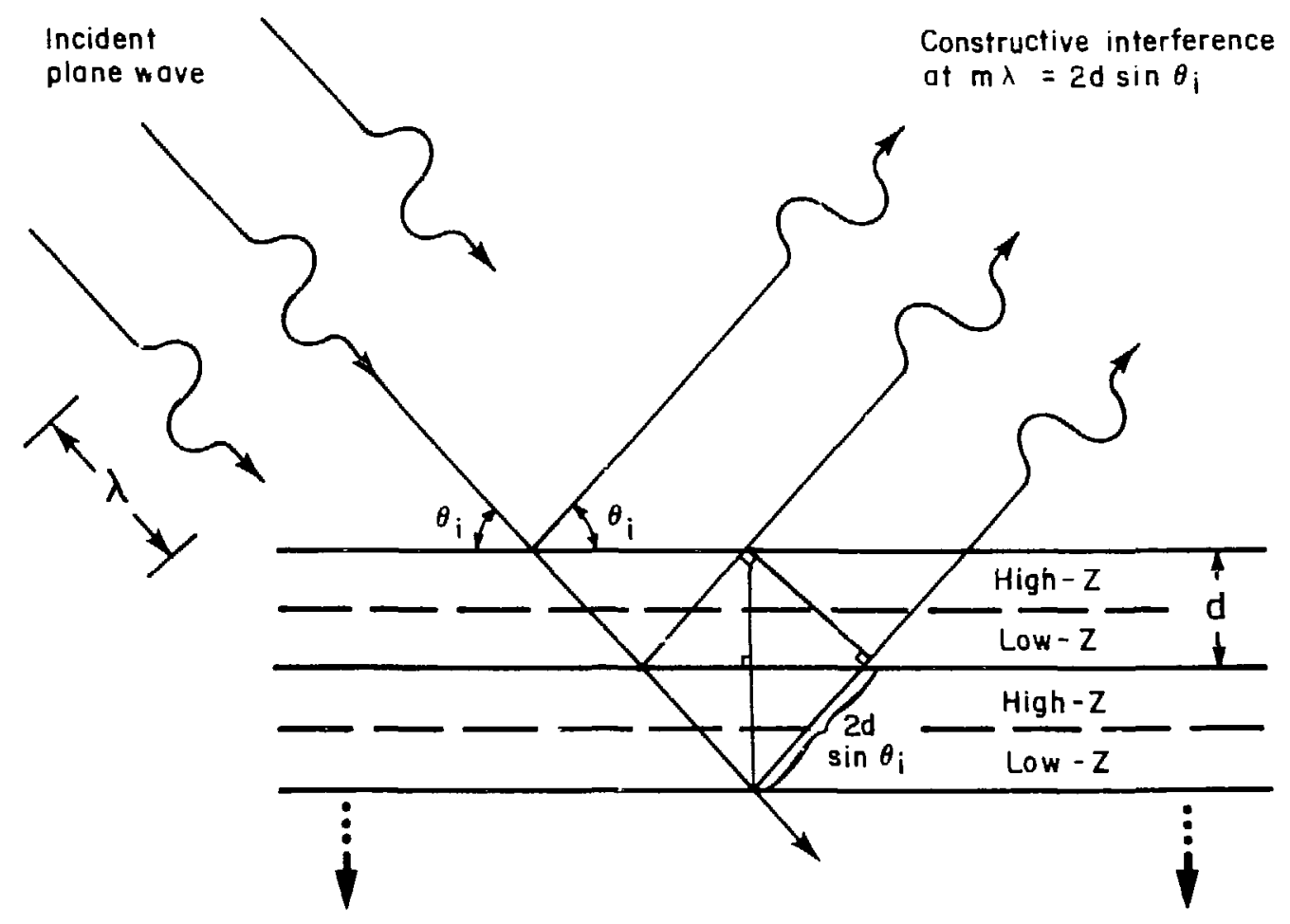

Fig. 3-6. Constructive interference of x-rays, scattered from the periodic multilayer structure, results in Bragg diffraction peaks at angles $\theta_{i}$ satisfying $m \lambda=2 d \operatorname{din} \theta_{i}$. The diffraction order is m and $\lambda$ is the $x$-ray wavelength. The structure period d is variable between $15 \AA$ and $200 \AA$. 
broad resolution in the multilayer is needed to provide higher diffracted flux, integrated over a spectral band of intermediate width.

The broadest multilayer spectral bands remain much narrower than reflector/filter channels and provide a less ambiguous spectral diagnostic with a greater number of data points available in the subkeV region.

Narrow energy resolution can be used effectively in continuouslydispersed (not energy-band) spectral measurements to observe structure on the spectral shape. To date, high resolution (E/ $\mathrm{E} \geq 100)$ has not been obtained in the subkev spectral region using metal multilayers.

There is great potential for engineering the diffraction properties of these devices to meet a variety of requirements. The number of layers sampled at a given wavelength may be enhanced using appropriate materials with low absorption properties. Improvements in the interface roughness will enhance peak reflectivity.13,16 Resolution may be broadened using graded d-spacings which decrease with distance from the surface. 12,13 Aspect ratios of the multilayer components and d-spacings can be varied not only with position in the stack but with two dimensional surface position as well. Metal multilayers may also be deposited on curved or bendable substrates (though not currently with the convenience with which molecular multilayers may be coated onto tightly radiused geometries) for a variety of imaging and dispersion schemes. 17,18

The immediate need of the LLNL laser-fusion program, at the time of the design of the metal-multilayer streaked spectrometer, was for an increased number of subkeV spectral points--more cleanly spectrally and temporally resolved. These intermediate resolution (E/ $\Delta E \sim 10)$ 
measurements were needed both to avoid late-time contributions to spectral shape which compromised time-integrated high-resolution grazinginciderice spectrometer measurements ${ }^{19}$ and to provide more complete and less ambiguous subkilovolt spectral coverage than was available with the reflector/filter spectral discrimination system on SX-1. At implementation, the system was slightly modified to look ror line emission from specific multilayered energy-transport targets described in Chapter 5. Two of the spectrometer channels were centered at characteristic emission energies of ionized cxygen and fluorine lines from $\mathrm{SiO}_{2}$ and teflon layers of the target.

\section{Spectrometer Design}

As a first effort, the metal multilayer spectrometer was designed as a five-channel narrow-energy-band spectral discrimination system. The lowest energy channel was positioned at $102-e V$ to verify the low-energy spectral roll-over which is characteristic of the anticipated semiPlankian spectrum. The second energy channel was positioned at $267-\mathrm{eV}$, near the expected spectral peak. Energy channels at $653-\mathrm{eV}, 737--\mathrm{E}$, and 943-eV were to measure the high-energy side of the spectral shape in the subkeV region.

Surface reflection. In addition to the Bragg peak, surface reflection from the synthetic crystal adds some background to the diffracted signal. As discussed above, this grazing-incidence surface reflection is efficient for a continuous range of photon energies below the criti$c a l$ energy $E_{c}$ determined by the grazing angle of incidence. Some reflection also occurs with lower efficiency about $E_{c}$. Large grazing 
angles reduce this background significantly at high energies. Relatively large Bragg angles, near $25^{\circ}$, were selected for this reason. However, the reflected signal (integrated over photon energy) can still be subsiantial compared with the narrow-energy-band diffracted signal. Measurements of reflectivity versus $2 \theta$ by Henke 20 and Underwood's 21 calculations of reflectivity at various photon energies corroborate this concern.

In order to avoid such unwanted low-energy contributions to the narrow-band-diffracted signal, thin-foil absorption filters were used to cut off the low-energy reflections. The k-absorption edges of a

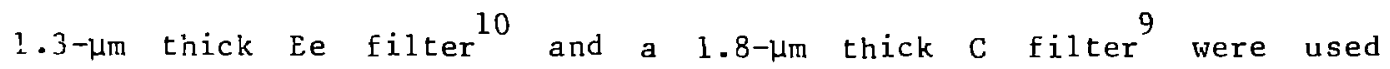
to minimize surface reflection background in the $102-\mathrm{eV}$ and $267-\mathrm{eV}$ channels respectively. The effectiveness of this isolation filter technique is illustrated in figire 3-7 where, in the first plot, the surface or Fresnel reflection baskground contributes to the Bragg diffraction of a $55-\AA$ d-spacing $\mathrm{Ti} / \mathrm{C}$ structure at $25^{\circ}$. This spectral signal, filtered by the carbon filter spectral transmission function in the second plot, results in a very clean and narrow spectral shape. The three higher-energy channels were filtered by $0.87-\mu m-t h i c k$ Al foil. 10

Metal-multilayer design. The d-spacing of the metal-multilayer structures which were fabricated was partially determined in this case by the Bragg angle which had previously been selected to meet constraints of low-energy reflection suppression and of instrument geometry and alignment. The required photon energy bands, which are selected for maximum transmission through the isolation filters or to match emission 


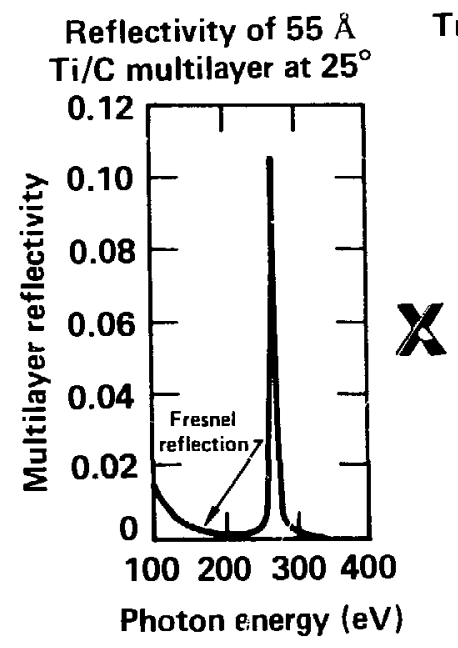

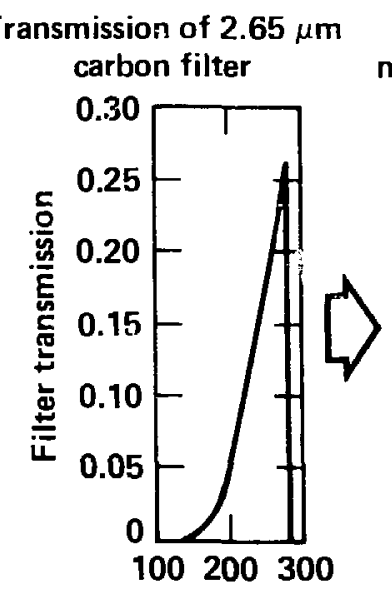

Photon energy (eV)
Efficiency of multilayer-filter combination

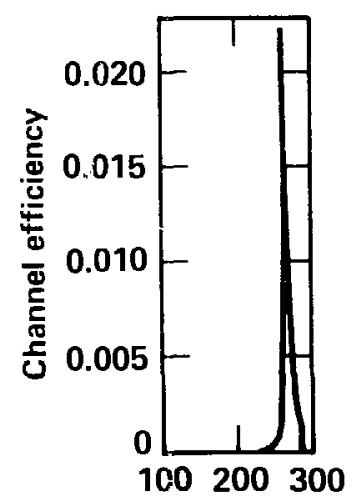

Photon energy (eV)

Fig. 3-7. Low-energy surface reflection contributions to the signal from a metal multilayer $x$-ray interference mirror may be eliminated using absorption filters. Here the Bragg peak is designed to lie under the carbon-filter transmission peak. A relatively large $25^{\circ}$ Bragg angle is used to suppress surface reflection. 
line energies, combine with the pre-selected Bragg angle to completely determine the d-spacing through equation $3-5$.

The component materials which form the layers of the structure are then selected to provide the needed index of refraction contrast and the desired $x$-ray transmission properties at the photon energy of interest. This selection is also constrained by sputtering (or vapor deposition) and interface characteristics of the materials. The metal-multilayer structures selected for a nominal $25^{\circ}$ (with exceptions noted) were 143- $d$-spacing $V / C$ for for $102 \mathrm{eV}, 55-\AA$ d-spacing $T i / C$ for $267 \mathrm{eV}$, 21- $\AA$-spacing $W / C$ at $27.1^{\circ}$ and at $23.8^{\circ}$ for $653 \mathrm{eV}$ and $737 \mathrm{eV}$ respectively, and $15-\AA \mathrm{d}$-spacing $\mathrm{W} / \mathrm{C}$ for $943 \mathrm{eV}$. Characteristics of these five multilayer structures are listed in Table $3-1$. Because of the precision achieved with a sputtering technique by Dr. Barbee at Stanford University, most of the structures were achieved with acceptable d-spacings in single runs. The V/C multilayer, designed for use at $102 \mathrm{eV}$, required several attempts, however, because of the uncertain knowledge of complex refractive index data at these very low and greatly unexplored $x$-ray energies. A reduction in refractive index from higher calibration energies resulted in a different effective d-spacing at 102 eV. The effective d-spacing $d_{\text {eff }}$ is obtained to first order accuracy in $\delta$ (where $\delta=1-n$, the decrement of the index of refraction $n$ ) by: 16

$$
d_{\text {eff }}=d_{0}\left(1-\delta / \sin ^{2} \theta_{i}\right)
$$

where $d_{0}$ is the actual d-spacing and $\theta$ is the Bragg angle. Consequently several iterations were required with d-spacing checks at the $B e-K_{\alpha}$ emission line performed by Dr. B. L. Henke's 1 lab $^{22}$ at the 
Table 3-1. Characteristics of Multilayer Interference Mirrors and Filter

\begin{tabular}{|c|c|c|c|c|c|}
\hline Channel Energy & $102 \mathrm{eV}$ & $267 \mathrm{eV}$ & $653 \mathrm{eV}$ & $737 \mathrm{eV}$ & $943 \mathrm{eV}$ \\
\hline Bragg Angle & $25^{\circ}$ & $25^{\circ}$ & $27.1^{\circ}$ & $23.8^{\circ}$ & $25^{\circ}$ \\
\hline d-spacing $(\AA)$ & $\begin{array}{l}178 \\
143 \AA)\end{array}$ & 55 & 21 & 21 & 15 \\
\hline $\begin{array}{l}\text { Scattering material/ } \\
\text { spacer }\end{array}$ & $\mathrm{V} / \mathrm{C}$ & $\mathrm{Ti} / \mathrm{C}$ & $\mathrm{W} / \mathrm{C}$ & $\mathrm{W} / \mathrm{C}$ & $\mathrm{W} / \mathrm{C}$ \\
\hline Width $\Delta E(\mathrm{eV})$ & 10 & 7 & 8 & 8 & 10 \\
\hline $\begin{array}{c}\text { Integrated reflec- } \\
\text { tivity (mrad) }\end{array}$ & 3.6 & 1.89 & 0.46 & 0.46 & 0.12 \\
\hline Peak reflectivity (\%) & 10.8 & 10.6 & 5.1 & 5.1 & 1.6 \\
\hline Filter material & $\mathrm{Be}$ & $\mathrm{C}$ & $\mathrm{A} 1$ & A1 & $\mathrm{Al}$ \\
\hline Filter thickness $(\mu \mathrm{m})$ & 1.3 & 1.8 & 0.87 & 0.87 & 0.87 \\
\hline
\end{tabular}


University of Hawaii. The actual d-spacing was $178 \AA$ for an effective d-spacing of $143 \mathrm{~A}$ at $102 \mathrm{eV}$ and $25^{\circ}$.

Metal-Multilayer Calibration

Each of the multilayer structures was calibrated for d-spacing, integrated reflectivity (over angle), and resolution $\Delta E$, at a cold emission line close to the photon energy of interest, as well as at several other emission lines. Peak reflectivity values $P$ are also available from these parameters. These calibrations, which are summarized in table 3-1, were performed in collaboration with Dr. B. L. Henke using fluorescent-line sources at his low-energy $x$-ray calibration facility at the University of Hawaii. 22

\section{Channel Response Calculation}

The absolute efficiency of each energy channel, in terms of photons converted to secondary electrons at the photocathode of the streak camera, may be obtained by folding the calibrated multilayer-diffraction efficiency with the calibrated filter-transmission efficiencies and the photocathode response.

The multilayer diffraction efficiency as a function of photon energy may be modeled as a Lorentzian of width $\Delta E$ and amplitude $P$. Both $\Delta E$ and $\mathrm{P}$ may be obtained from Henke's measurements.

Filter foil thicknesses are measured to $\pm 10 \%$. The filter transmission effiriencies are then obtained using tables of measured massabsorption coefficients.

The photocathode response is approximated from absolute measurements by Henke et. al. ${ }^{23}$ interpolated with an $\operatorname{E\mu (E)}$ fit, where $E$ is the photon energy and $\mu(E)$ is the photon-energy dependent mass-absorption 
coefficient. Low-flux levels in the low-Z energy transport measurements described in Chapter 5 necessitated the use of a $1100-\AA$ CsI transmission photocathode. 23,24 other measurements using a CsI photocathode with this streaked multilayer spectrometer are presented in Ref. 25. Again these photocathodes are vacuum deposited on $0.25-\mu m \pm 10 \%$ thick carbon foils. ${ }^{9}$

The spectral shapes resulting from these combined factors are shown together in figure 3-8. The low-energy lobe apparent on the 102-eV channel is the result of high surface reflectivity from $V / C$ at that energy, which is only partially reduced by the Be filter.

Spectrometer Assembly and Alignment

The metal-multilayer spectrometer system was initially intended to operate with a $25^{\circ}$ Bragg angle for all of the multilayers. This design would allow the five synthetic (one dimensional) crystals to be coplanar, enhancing the ease of alignment. In this side-by-side ar$x$ angement, the multilayers were trimmed to 1.5 -mm wide strips to allow all five channels to simultaneously illuminate the 13-mm long photocathode without overlap (figure 3-9). Different source-tomultilayer distances could be accommodated by this configuration without altering the multilayer mounting hardware. Once bench aligned, a coplanar system could be adjusted as a single unit. This inital design was modified in anticipation of observing emission lines from ionized $\mathrm{SiO}_{2}$ and teflon (CF) layers of an energy transport target at $653 \mathrm{eV}$ and $737 \in V$ respectively. Because the required angles of $27.1^{\circ}$ and $23.8^{\circ}$ are close to the initial $25^{\circ}$, a modification of the design was possible which retained the initial alignment advantages. This design 
Five narrow-band synthetic crystal channels

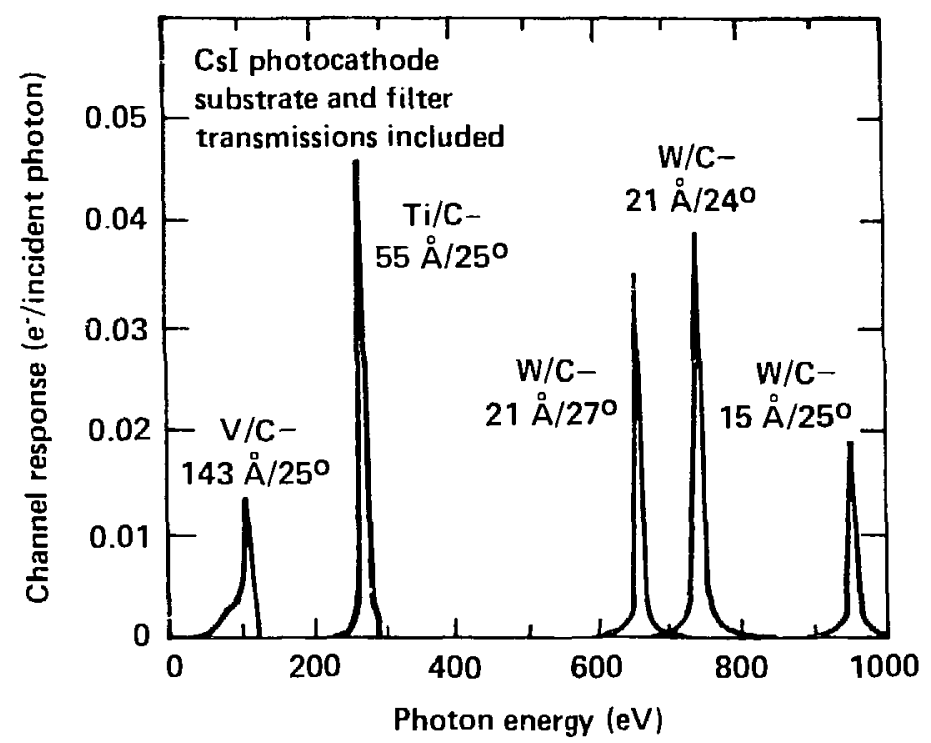

Fig. 3-8. Five narrow-band metal-multilayer channels for time-resolved subkeV x-ray diagnostics. Contributions to these spectral window shapes include calibrated diffraction characteristics of the multilayers, calculated surface reflection, calibrated transmission characteristics of filters and photocathode substrate, and the efficiency of the 100-A thick CsI photocathode. 


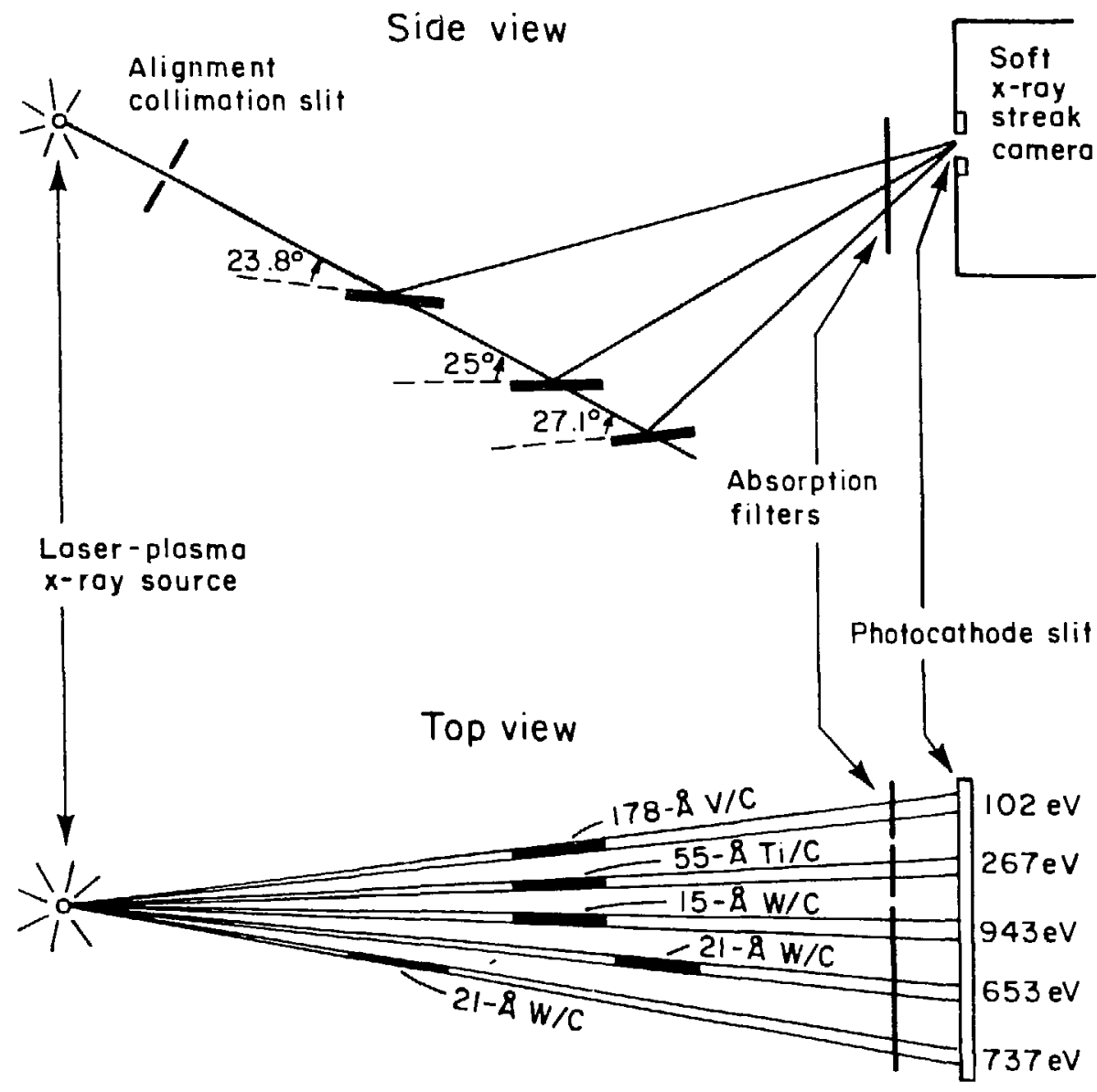

- Mefal-multilayer x-ray

interference mirrors

Fig. 3-9. Metal-multilayer spectrometer configuration. The 1.5-mm wide multilayer strips are positioned along an $x$-ray emission beam such that all reflect at the predetermined s.igle, through filter foils and onto adjacent sections of the streak carera photocathode. The collimation slit is removed after alignment. 
is schematically displayed in figure 3-9. The three $25^{\circ}$ multilayer strips remained coplanar while the other two multilayer strips were positioned along the same line of sight (though displaced out of the plane of incidence) such that they reflect the same incident beam onto the photocathode slit of the soft $x$-ray streak camera.

The system was bench aligned and the multilayers were fixed into position with respect to each other. The system was then positioned with respect to the source and streak camera using a $c w$, He-Ne laser beam which converged through a $300-\mu m$ focal waist at the target position and diverged from there to the multilayer system, illuminating all of the multilayer strips. A collimator slit, across the multilayer array, allowed a narrow beam to project across the center of each multilayer strip. The multilayer system, positioned at a set, predetermined distance from the photocathode, was then oriented to reflect the five strips of light onto the photocathode slit. Once aligned, the alignment collimation slit was removed. The $100-\mu \mathrm{m}$ wide photocathode slit provides collimation beyond the alignment precision needed for data acquisition.

Streaked measurements, with out filters, were used to specify the position of the diffracted beams at the photocathode slit and thus determine the necessary filter positions. Filters are positioned immediately in front of the photocathode (figure 3-9) to obscure any stray scatter from the spectrometer apparatus. 


\section{Summary}

In increasing degrees of refinement, absorption-edge filters, filters with grazing-angle $x$-ray mirrors, and metal multilayer $x$-ray interference mirrors have been used for spectral definition. Grazingangle reflectors have allowed energy resolution $E / \Delta E$ of between 3 and 5 to be obtained with absorption-edge filters below the spectral emission peak. Energy bands of $210 \mathrm{eV}$ are available and have been implemented with sputtered metal multilayers. 


\section{$\underline{\text { References }}$}

1. K. G. Tirsell, H. N. Kornblum, V. W. Slivinsky, Bull. A:..ər. Phys. Soc. 23, 807 (1978); Lawrence Livermore lvational Laboratory Report UCRL-81378, 1979 (unpubl ished).

2. R. H. Day, P. Lee, E. B. Saloman, and D. J. Nagel, Los Alamos National Laboratory Report, LA-7941-MS, 1981 (unpublished).

3. Victor Rehn, Low Energy X-Ray Diagnostics - 1981, edited by D. T. Attwood and B. L. Henke (AIP, New York, 1981), p. 162 .

4. J. D. Jackson, Classical Electrodynamics, 2nd ed. (Wiley, New Ycrk, 1975), P.285.

5. Ibid., P. 279 .

6. A. Toor and H. F. Finn, computer code REFLECT2 (Lawrence Livermore National Laboratory, Livermore, Cal.). The photoionization cross sections for subkilovolt \& rays used by this code are from B. L. Henke, University of Hawaii. The cross sections for higher energy $x$ rays are from the LLNL cross section library file cSLOW $3 / 77$.

7. G. L. Stradling, D. T. Attwood, J. W. Houghton, E. L. Pierce, and D. P. Gaines, Bull. Am. Phys. 23, 880 (1978).

8. R. L. Kauffman, G. L. Stradling, and D. T. Attwood, Bull. Am. Phys. Scc. $\underline{23}, 880(1978)$.

9. Arizona Carbon Foil Co., Tucson, Ariz.

10. Lebow Co., Goleta, Ca.

11. R. L. Kauffman, G. L. Stradling, E. L. Pierce, and H. Medecki, Low Energy X-Ray Diagnostics - 1981, edited by D. T. Attwood and B. L. Henke (AIP, New York, 1981), p. 65.

12. E. Spiller, Appl. Opt. 15, 2333 (1976); Appl. Phys. Lett. 20, 365 (1972).

13. E. Spiller, Low Energy X-Ray Diagnostics - 1981, edited by D. T. Attwood and B. L. Henke (AIP, New York, 1981), P. 124.

14. T. W. Barbee, Jr., Low Energy X-Ray Diagnostics - 1981, edited by D. T. Attwood and B. L. Henke (AIP, New York, 1981), P. 131; T. W. Barbee, Jr., and D. C. Keith, Stanford Linear Accelerator Center, SSRL Report No. 78/04, III-26 (1978).

15. B. L. Henke and M. A. Tester, Advances in X-Ray Analysis, Vol. 18 (Plenum Press, New York, 1975), P. 76; Low Energy X-Ray Diagnostics - 1981, edited by D. T. Attwood and B. L. Henke (AIP, New York, 1981), P. 85 . 
16. J. H. Underwood and T. W. Barbee, Jr., Low Energy X-Ray Diagnostics - 1981, edited by D. T. Attwood and B. L. Henke (AIP, New York, 1981), p. 170 .

17. B. L. Henke and T. J. Tanaka, Bull. Am. Phys. Soc. 26, 992 (1981).

18. J. P. Henry, E. Spiller, and M. Weiskopf, Proceedings of the Brookhaven Conference on High Resolution Soft X-Ray Optics, Brookhaven National Lab., New York, Nov. 1981 (in press).

19. D. Matthews, private communication.

20. B. L. Henke, private communication.

21. J. H. Underwood, private communication.

22. B. L. Hen'se and E. S. Ebisu, Advances in X-Ray Analysis, edited by C. L. Grant, C. S. Barrett, J. B. Newkirk, and C. O. Ruud (Plenum, New York, 1974), Vol. 17, pp. 150-213.

23. B. L. Henke, J. P. Knauer, and K. Permeratna, J. Appl. Phys. 52, 1509 (181).

24. G. L. Stradling, H. Medecki, R. L. Kauffman, D. T. Attwood: and B. L. Henke, Appl. Phys. Lett. 37, 782 (1980).

25. G. L. Stradling, T. W. Barbee, Jr., B. L. Henke, E. M. Camptell, and W. C. Mead, Low Energy X-Ray Diagnostics - 1981, edited by $D$. T. At twood and B. L. Henke (AIP, New York, 1981), p. 292. 
Chapter 4

DYNAMIC SOFT X-RAY STREAK CAMERA AND PHOTOCATHODE

CHARACTERIZATION: SWEEP-GPEED, SWEEP LINEARITY, DYNAMIC RANGE, CSI AND Au PHOTOCATHODES

\section{Introduction}

As with all diagnostic instruments, careful characterization is a necessary preliminary to reliable, credible data aquisition and analysis. The relative importance of various streak camera parameters is application dependent; however the unique characteristics of high temporal resolution and shot-noise limited sensitivity as well as sweep linearity, large dynamic range, 1 and the number of channels which can be continuously recorded across the photocathode are all important parameters. The evaluation of these characteristics is addressed in this chapter. Absolute spectral sensitivity, ${ }^{2}$ although a very important area of evaluation, will not be discussed in detail.

The optimization of photocathodes for both fast and ultra-fast $x$-ray detectors is an area of active interest in the $x$-ray diagnostic conmunity.3-11 $\mathrm{Au}$ and CsI transmission-photocathode evaluations in side-byside in situ measurements are reported here. The temporal response and sensitivity of the two materials are compared. Photocathodes were evaluated over a wide range of thicknesses.

\section{Pulsed X-ray Source: The Monojoule-Laser Irradiation Lab}

Very short ( 2 70-psec), intense $x$-ray bursts are needed for dynamic characterization of the soft $x$-ray streak camera. Such signals may be obtained from laser-produced high-Z plasmas. Irradiation conditions greater than $10^{13}-10^{14} \mathrm{~W} / \mathrm{cm}^{2}$ intensity with a few joules of $1.06-\mu_{m}$ light delivered in $50 \mathrm{psec}$ or less are very satisfactory for the production of such plasmas. In the LLNL laser fusion program, the Monojoule-laser irradiation 1 ab is used for such pulsed $x$-ray diagnostic testing and development.

The Monojoule laser is a neodymium:YAG laser capable of producing single laser pulses with energies between one and three joules in 50-psec or 150-psec laser pulse widths, respectively. The laser oper- 
ates with a passively mode-locked Nd:YAG oscillator. Mode-locking and $Q$ switching are done with a saturable absorber dye cell. A 1-mJ pulse is switched out near the peak of the oscillator wave train by a nitrogen spark gap. The oscillator pulse passes through two 3/8-inch YAG preamplifiers, a 5/8-inch glass amplifier and a one-inch glass amplifier as shown in figure 4-1. This amplification process raises the pulse energy as high as three joules. 12

For x-ray streak camera sweepspeed calibrations, the laser pulse is directed through a variable-spacing etalon to produce a series of optical pulses, typically 200-500 psec apart, with each pulse having nominally one-half the energy of the preceding pulse. X-ray pulse-trains are produced by irradiating a high-Z slab target with the laser pulsetrain, in an evacuated $\left(10^{-4}-10^{-5}\right.$ Torr $)$ target chamber. In fullpower operation the Monojoule laser can fire with a three-minute repetition period for efficient calibration and testing procedures.

The configuration of the etalon, target chamber, target and soft x-ray streak camera are shown schematically in figure 4-2. A matter of interest is that the physical spacing between laser pulses 500 psec apart is $15 \mathrm{~cm}$. This pulse spacing is obtained with a $7.5 \mathrm{~cm}$ spacing between mirrors in the etalon. The source-to-flange distance of the target chamber is $30.5 \mathrm{~cm}$. Without the $\mathrm{x}$-ray mirror apparatus, the streak-camera photocathode may be positioned $53 \mathrm{~cm}$ from the source.

Triggering of the soft $x$-ray streak camera is accomplished by diverting part of the laser oscillator pulse directly to a photodiode. The photodiode generates an electrical pulse which triggers the streak camera's avalanche-transistor sweep circuit of the streak camera. Path length traveled by the optical/electrical pulse controls the trigger 


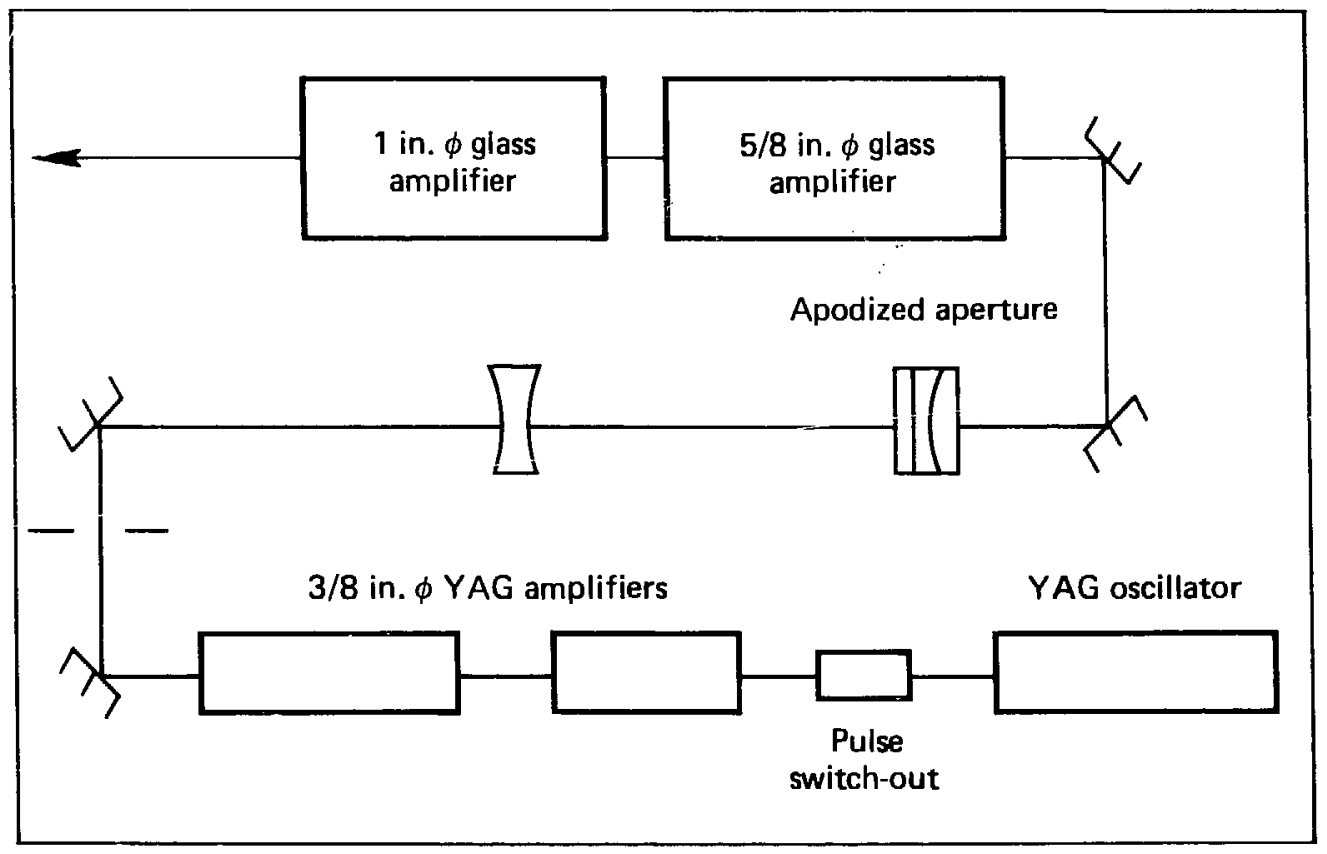

Fig. 4-1. The Monojoule laser, a 50-psec, 1 to 3 joule, neodymium:YAG laser used for diagnostic testing and development. 


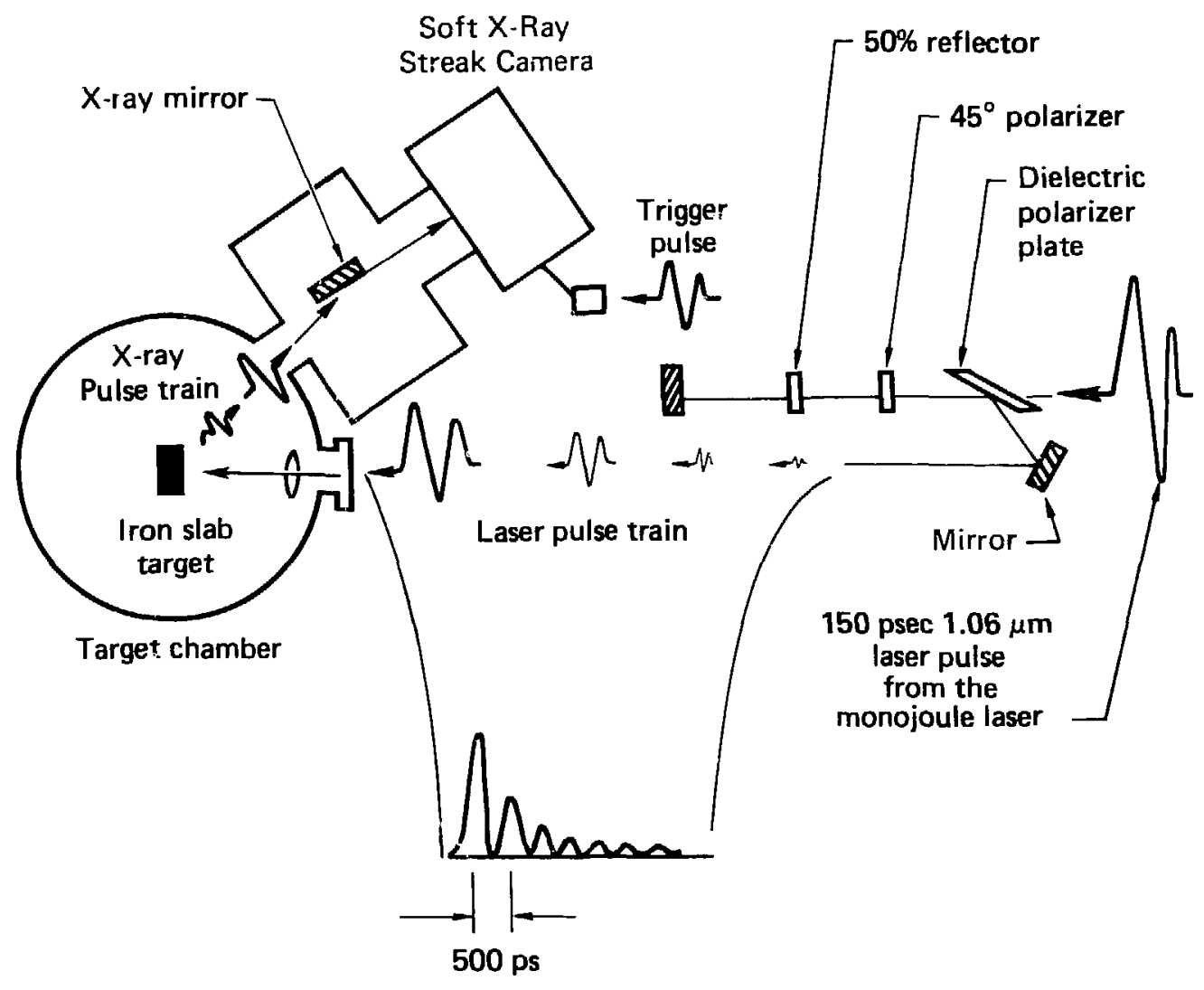

Fig. 4-2. The Monojoule target chamber configuration for calibration and experiments. A 500-psec etalon splits the 1.06- $\mu \mathrm{m}$ laser pulse into a pulse train which heats the iron-slab target and produces a train of $x$-ray pulses. The $x$-ray pulse-train is reflected by an x-ray mirror onto the streak camera slit. 
timing relative to the $x$-ray pulse train incident on the streak camera photocathode.

\section{Temporal Calibration}

The soft $x$-ray streak camera temporal-calibration procedure consists of recording a string of $x$-ray pulses with known temporal spacing. The resultant streak camera data may then be routinely reduced and analyzed to show sweep-speed, sweep linearity, dynamic range, and photocathode uniformity.

Figure 4-3 is the photographic record of an x-ray pulse-train. These data were recorded without filtering the x-ray signal. The incident laser pulses were 150-psec wide FWHM and separated by 500 psec.

The data are taken on Koday Royal-X Pan film for maximum sensitivity and resolution. After the data are recorded and before the film is developed, a strip at the edge of the film is exposed to a stepwedge which is chromatically matched to the image-intensifier phosphor (figure 4-3). The film is then developed for 4.5 minutes in Kodak DK-50 deve1oper. This development procedure raises the film speed to ASA 2000 and reduces the contrast of the film to allow a large dynamic range to be recorded. Intensity-versus-position is extracted using a computercontrolled micro-densitometer. The stepwedge data are interpolated using a polynomial fit to produce a D-vs-logE calibration curve, characteristic of both the particular development and piece of film (figure 4-4). The density data are then unfolded with the D-logE curve to produce intensity-versus-position information. A detailed discussion of the data reduction and analysis is undertaken in the Appendix. 


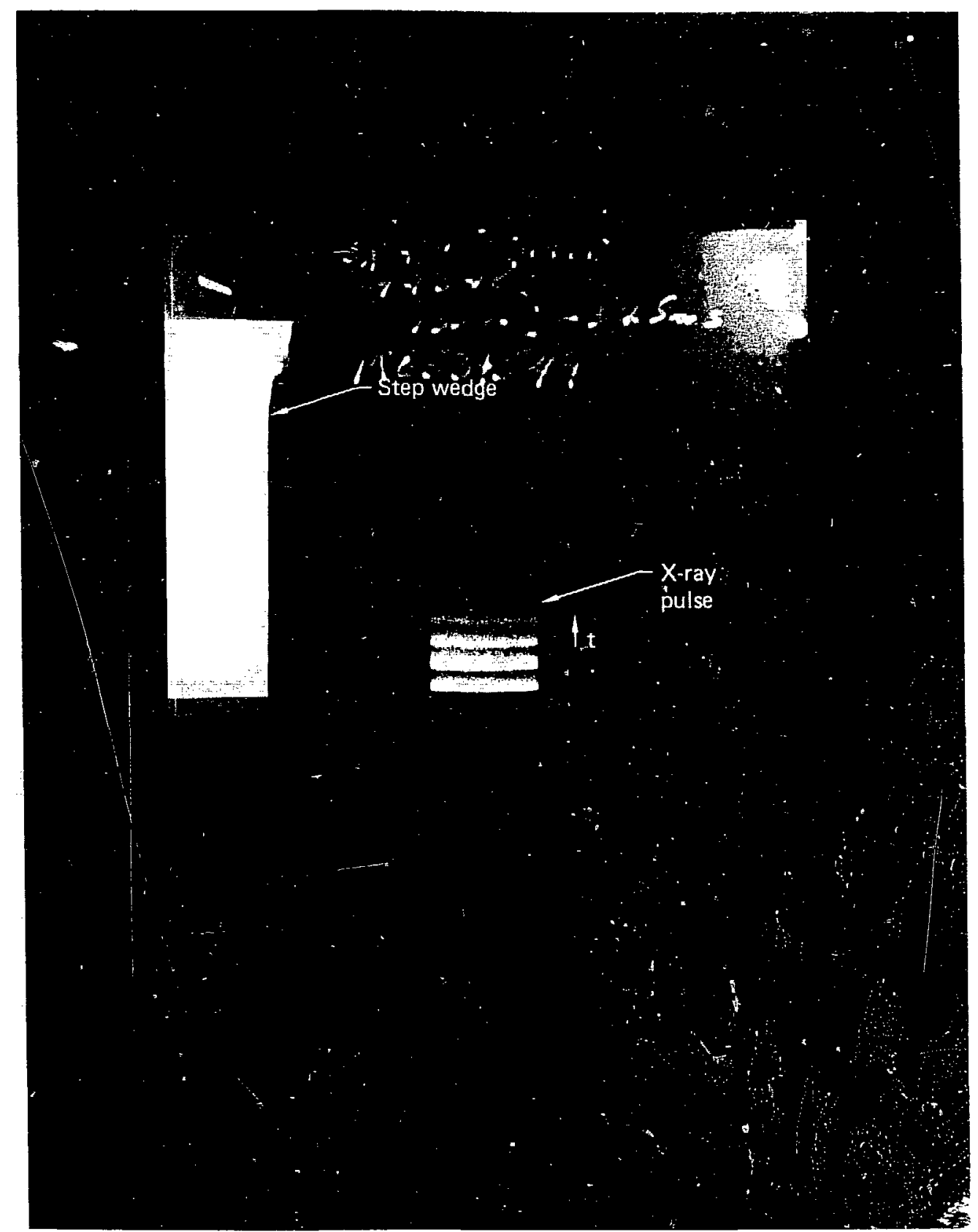

Fig. 4-3. Soft $x$-ray streak camera calibration data. The streaked $x$-ray pulse-train image is exposed on the center of the Kodak Royal-X Pan film. A step wedge is exposed on the edge of each film to permit a density-versus-intensity reduction of the recorded data. 


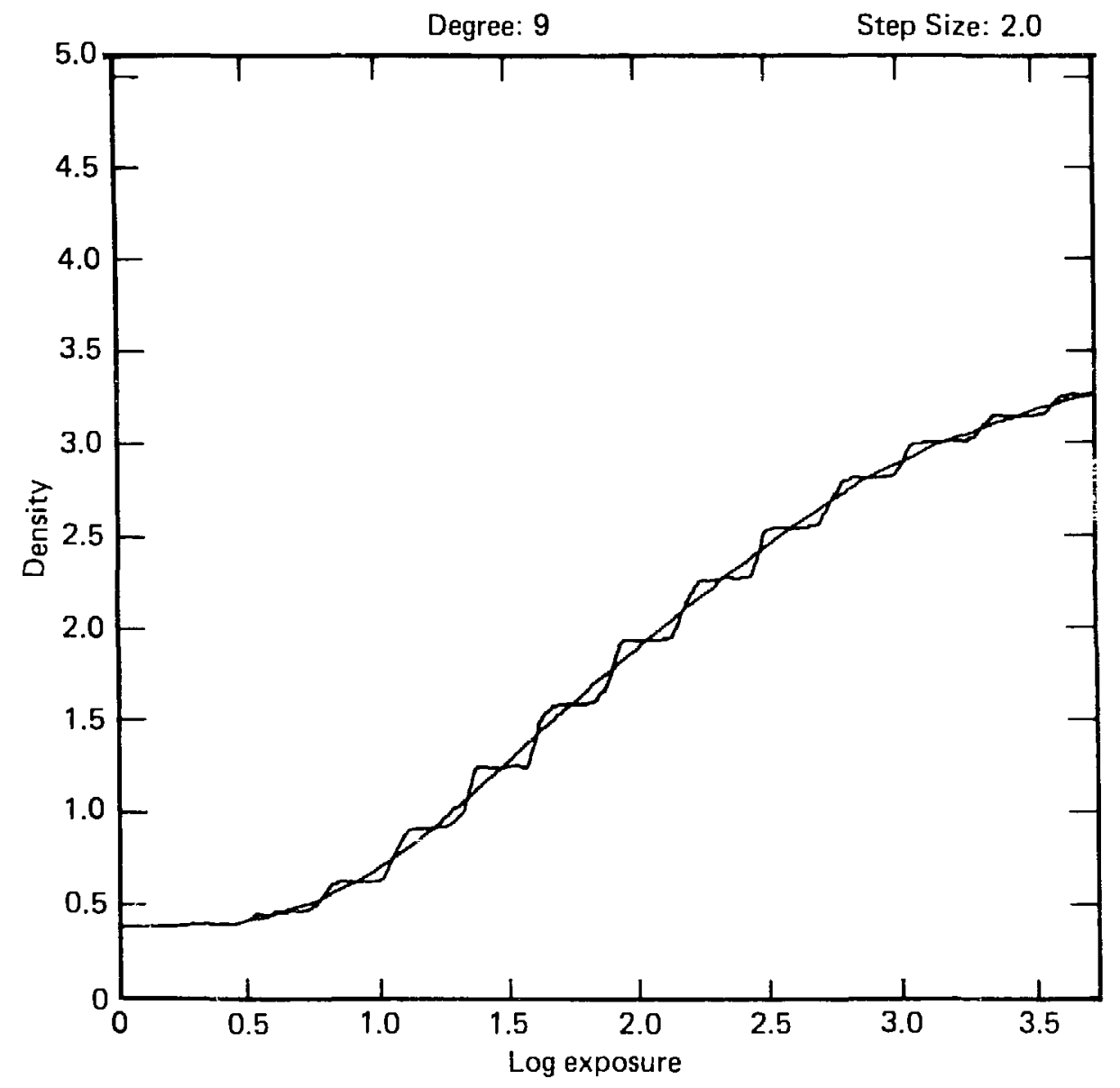

Fig. 4-4. D-vs-LogE characteristic curve of the film, a 9-degree polynomial $\mathrm{fit}$ to step-wedge data. 
The spatial separation of intensity peaks divided by the etaloncontrolled temporal separation of the pulses (500 psec) shcws the SX-1 camera's inverse sweep-speed with a X3 sweep card to be nominally 134 $\mathrm{psec} / \mathrm{mm} \pm 10 \%$. Sweep linearity is deduced from comparison of the spatial pulse separations along the pulse train.

The $50-\mu \mathrm{g} / \mathrm{cm}^{2}$ carbon substrate with a $300-\AA$ gold photocathode was seen to be extremely uniform across the slit. Figure $4-5$ is the cathode response-versus-slit position information taken from the data shown in figure 4-3.

Dynamic range is a function of resolution, pulse width, and other camera operating parameters. Detailed dynamic range studies with the LLNL optical streak camera, ${ }^{l}$ which is very similar to the soft $x$-ray streak camera discussed here, have demonstrated a dynamic range of $210^{3}$. Comparisons with $x$-ray diodes ${ }^{2}$ have shown the soft $x$-ray streak camera to have a linear range exceeding $\sim_{10}^{2}$. For the temporal-resolution region of interest here, a dynamic range of greater than one chousand may be expected and will be evident in the data presented. Full saturation was not seen with laser-pulse energies on an iron slab target as high as $800 \mathrm{~mJ}$. X-ray pulses generated by laser energies as low as $4 \mathrm{~mJ}$ were easily discernible above the noise level.

\section{Evaluation and Comparison of $\mathrm{Au}$ and $\mathrm{CsI}$}

\section{Transmission Photocathodes}

Thin ( $~ 100 \AA$ ) Au films are commonly used for $x$-ray-streak-camera transmission photocathodes. However, in some applications such as $x$-ray backlighting or time-resolved crystal spectroscopy, insufficient x-ray flux can limit the effectiveness of the streak camera. Also, as 


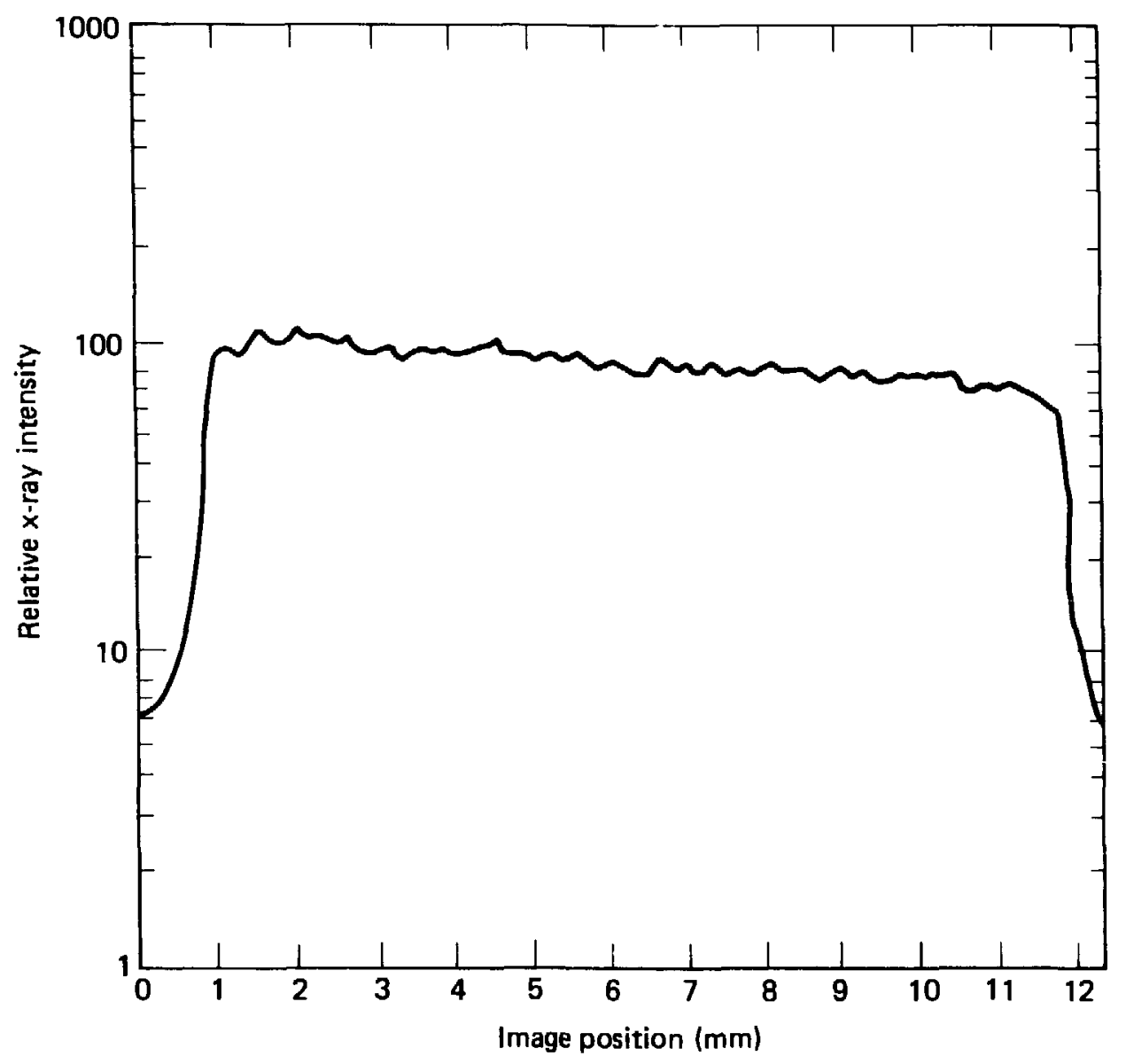

Fig. 4-5. An intensity-versus-position scan across the photocathode slit of soft $x$-ray streak camera calibration data showing the uniformity of the photocathode and of the photocathode substrate. 
discussed in Chapter 2, the secondary-electron energy spread from Au is too large to optimize temporal resolution. An improvement in the sensitivity and a reduction in the secondary-electron energy spread of the streak-camera photocathode can thus extend the range of application of the instrument. Considerable international attention has been given to the development of improved-sensitivity $x$-ray photocathodes. ${ }^{3-11}$ Henke, et. a1. ${ }^{9}$ have reported dc measurements of photocathode efficiencies for a variety of conductors and insulators. Of particular interest is CsI, with a photocathode efficiency greater than that of Au by a factor of ten at $573 \mathrm{ev}, 11$ and a factor of thirty at $1487 \mathrm{ev}$. Broad maxima in the sensitivity-veraus-thickness response curves of both CsI and Au transmission photocathodes have been measured. 11 The optimum photocathode thicknesses of $100 \AA$ for $A u$ and $1100 \AA$ for CsI are set primarily by secondary electron mean-free-paths and work functions in the respective materials. The dc measurements also give the secondary-electron energy spreads of $\mathrm{CsI}$ and $\mathrm{Au}$ as $1.6 \mathrm{eV}$ and 3.95 eV respectively, indicating that the electron transit time spreading should be smaller in CsI than in Au, thus improving temporal resolution in x-ray streak cameras. 11 However, potential problems which may arise from pulsed operation of CsI in a streak camera are not tested in the dc experiments. One example of possible pulsed-mode difficulties would re loss of temporal resolution due to late-time emission (straggling) of slow electrons. Here over-all photocathode performance in a streak camera environment is checked with typical picosecond-scale pulsed signals. 
Side-By-Side Comparisons

The soft $x$-ray streak camera is equipped with a demountable cathode assembly allowing convenient replacement. Simultaneous comparisons are possible of different materials and thicknesses using a composite arrangement which has up to six components across a single substrate. The composite photocathodes consist of samples of CsI or Au of different thicknesses, evaporation deposited at different positions across the thin carbon-foil substrate. Figure $4-6$ is a typical streaked image of the response of a six-step composita photocathode to a 270 -psec $x$-ray pulse. Three components are different thicknesses of Au: $70 \AA, 170$ $\AA$, and $440 \AA$, and the other three components are CsI: $530 \AA, 1120$ $\AA$, and $1540 \AA$.

This data is averaged across the spatial extent of each channel, and converted to $x$-ray intensity to produce temporal profiles for each component of the composite photocathode.

The pulsed $x$-ray source used for these side-by-side measurements was a laser-heated 8-um-thick titanium ta:get. The $x$-ray flux was filtered using $x$-ray absorbers and reflecters to define a modestly narrow $(E / \Delta E \sim 5)$ x-ray energy channel. A 4000- $\AA$ vanadium foil and a $3^{\circ}$ nickel x-ray reflector are used to form a spectral channel between $400 \mathrm{eV}$ and $500 \mathrm{eV}$. The $x$-ray pulse width, which varies significantly with channel energy, was measured to be $270 \mathrm{psec}$ FWHM. Curved crystal spectroscopy $^{13}$ shows that $L_{\alpha, \beta}$ lines of ionized titanium contribute significantly to the detected channel. 


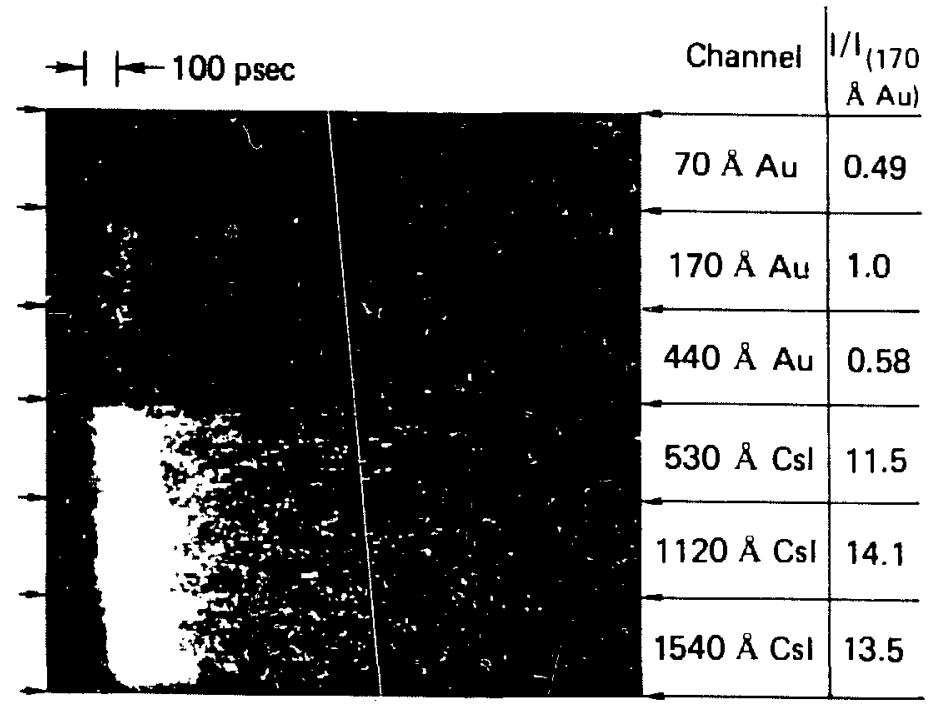

Time -

Fig. 4-6. Streaked intensity-versus-time data obtained using a sixcomponent composite photocathode. This technique determines the relative response of samples consisting of different material/thickness combinations in simultaneous, side-by-side operation in the streak camera environment. The incident $x$-ray pulse-width is 270 psec. The peak intensity of each channe? relative to the 170-A-Au channel peak intensity is given in the table. 
Temporal response characteristics. Figure $1-7$ gives the relative

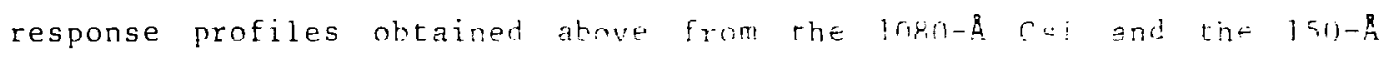
Au channels of data from a composite photucathode where the incident sigial was a train of $270-p s e c$ r-ray pulses spaced inn-psec apart. These two channels represent optimum thickness of holh materials. The response curves confirm the greater CsI sensitivily and show its temporal response to be comparable to that of Au for this pulse width. CsI should show even greater sensitivity 11 above its iodine ahsurptiun edge at $630 \mathrm{eV}$. The temporal profiles of both materials are nearly identical down to $1 / 30$ of the peak intensity for each incident pulse, at which point the CsI curve begins to exhibit a low-intensity tail. The late time tail shown in figure 4-7 was not apparent in data taken with some CsI photocathodes. This late-time tail may indicate some electron straggling from the CsI that is not present in Au, although other possibilities have not been discounted. The CsI tail was not as apparent in other CsI/Au comparison measurements. The phenonenon may have a parametric dependence on flux level and pulse length. Further investigation of the csi temporal response characteristics is warranted. The large dynamic range of the soft $x$-ray streak camera is apparent in figure $4-7$.

The CsI and Au photocathodes which we tested range in chickness from $50 \AA$ ํ to $4000 \AA$. The relative sensitivities of all of these photocathodes are correlated using component samples common to different composite photocathodes to scale their response levels. The correlated relative intensities of both materials, for a nominal x-ray energy of $500 \mathrm{eV}$ as described above, are plotted as a functior. of thickness in figure 4-8. The data points in the figure represent the relative 


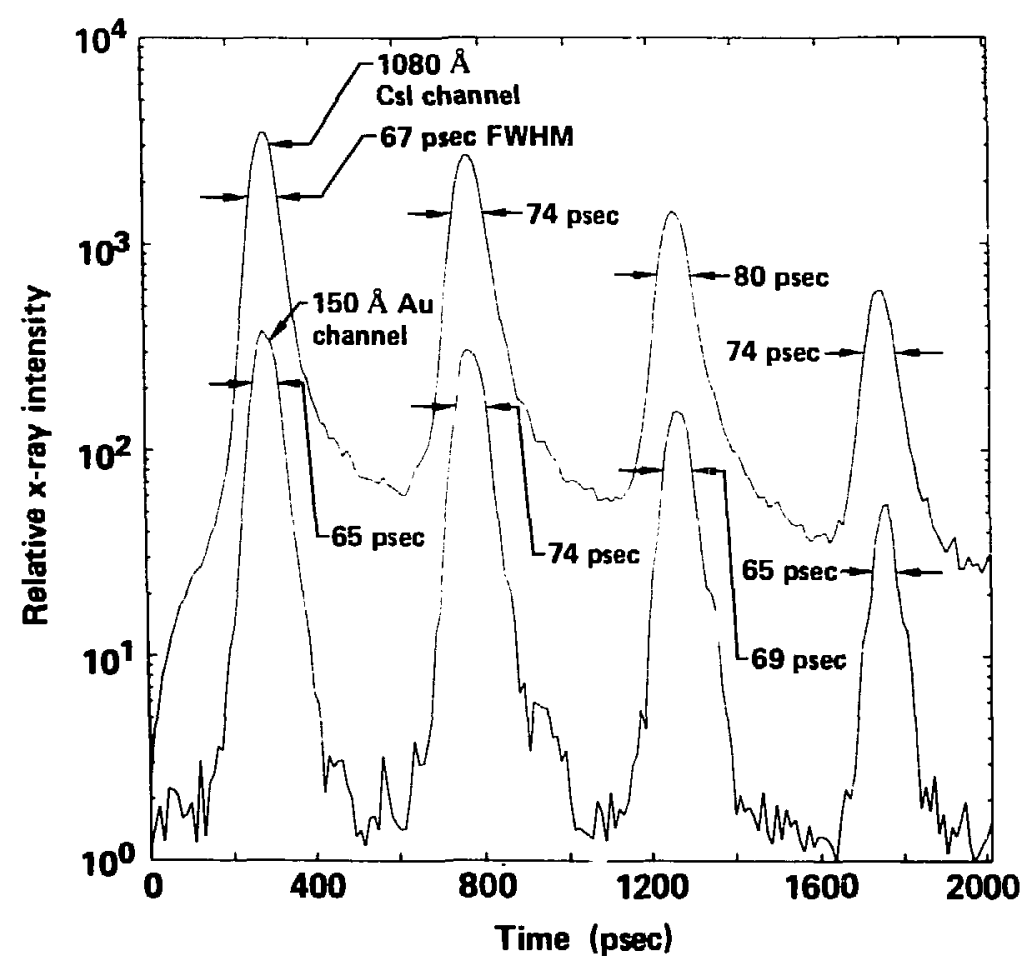

Fig. 4-7. Temporal profiles of the 1080-A CsI and the 150-A Au channels of a composite photocathode response to a train of $270-p s e c$ $x$-ray pulses spaced 500 psec apart. The temporal response of CsI is seen to match that of Au with nearly ioentical shapes for each pulse down to $\mathrm{I} / 30$ of the peak pulse intensity. 


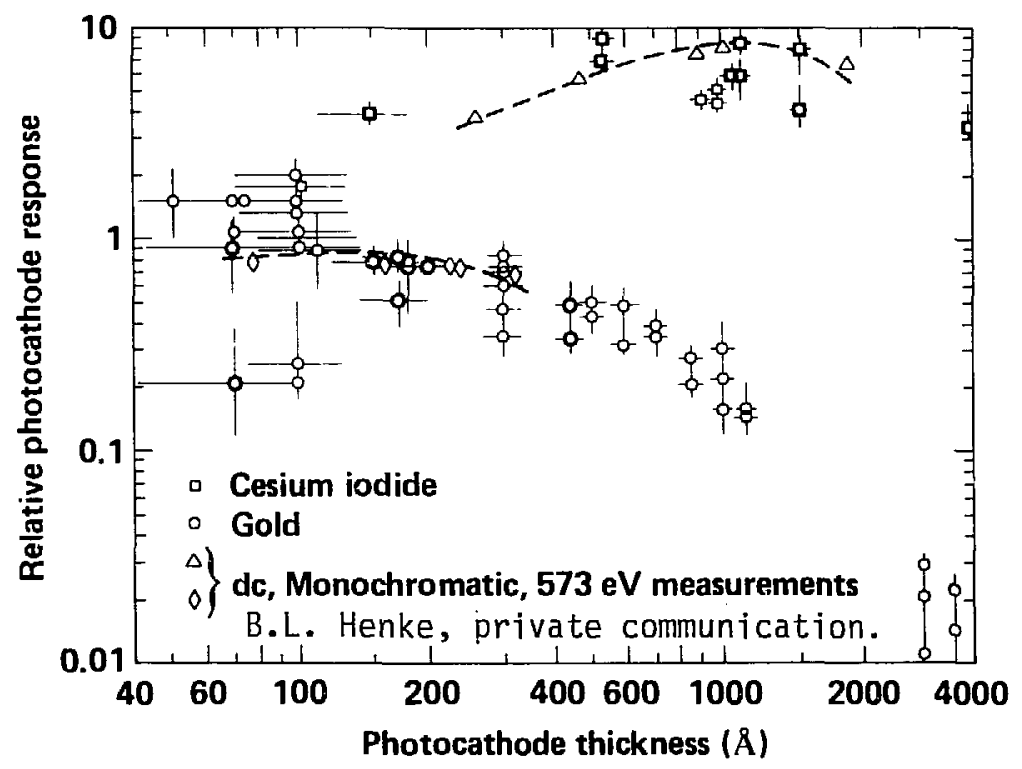

Fig. 4-8. Relative response-versus-thickness of CsI and Au to $2500-e V$ excitation. The data points represent the relative-response measurement of individual samples of a given material thickness combination. The vertical bars indicate the shot-to-shot spread of each sample response. The horizontal bars show the measurement uncertainty ( $30 \AA$ ) in sample thickness. Also plotted are previously measured intensity-versusthickness data, taken with dc, monochromatic, 573-eV excitation, which have been scaled for comparison. 
response measurement of individual samples of a given material-thickness combination. The vertical bars indicate the shot-to-shot spread $n$ the response of each sample. These variations stem from fluctuations in the target emission spectrum and from camera noise. The range of flux levels required to measure the span of component sensitivities on several composite photocathodes also produced variations when camera dynamic range limitations were exceeded. The horizontal bars show the measurement uncertainty $( \pm 30 \AA)$ in photocathode thicknesses.

Very thin ( $100 \AA)$ Au samples show a wide variation in sensitivity. This variation is seen among thin Au samples from any of the several evaporation facilities used and even among samples produced in the same evaporation run. Such a lack of consistency in thin photocathode production may be due to variable surface properties, uncertainties in thickness measurements, or other unknown effects characteristic of very-thin film deposition. Because of this lack of thin sample reproducibility, $200 \AA$ is recommended as a minimum thickness for Au photocathodes. Also plotted in figure 4-8 are the previously measured dc intensity-versus-thickness data taken by Henke, 11 obtained with a dc, monochromatic, 573-ev excitation, which have been scaled for comparison with these meas:ırements. Sensitivity-versusthickness maxima, which correspond well with Henke's data, are observed in the streak camera measurements. The $1100-\AA$ thick $C s I$ is seen to be a factor of five more sensitive to $400-e V$ - $500-e V$ x rays than $200-\AA$ Au. The difference in the sensitivity of CsI relative to $A u$ between the two sets of data may be attributable to the mix of $x$-ray energies in the pulse source. 
CSI photocathodes are seen to significantly increase $x$-ray streak camera sensitivity. The late-time tail in CsI emission may limit the dynamic range of the photocathode to 230 . Such effects require further study to examine their consistency, as well as their dependence on signal strength and pulse duration. CsI proved very useful for the narrow band, low laser-energy, low-Z measurements discussed in chapter 5. The metal multilayer reflective efficiencies and photon-energy pass-bands reduced signal levels at the instrument to below the Au photocathode detection level. A CsI photocathode provided the necessary margin of sensitivity. The LLNL laser-fusion program is also implementing these new photocathodes in other selected applications where increased sensitivity is required. 


\section{References}

1. S. W. Thomas and G. E. Phillips, in Proceedings of the 13th international Congress on High Speed Photography and Photonics, edited by Shin-Ichi Hyodo (Japan Society of Precision Engineering, Tokyo, Japan, 1979), p. 471; S. N. Thomas, Ibid., P. 838.

2. R. L. Kauffman, G. L. Stradling, E. L. Pierce, and H. Medecki, Low Energy X-Ray Diagnostics - 1981, edited by D. T. Attwood and B. L. Henke (AIP, New York, 1981), P. 66.

3. M. Ya. Schelev, and V. A. Chevokin, private communication, (1978).

4. D. J. Bradley, A. G. Roddie, W. Sibbett, M. H. Key, M. J. Lamb, C. L. S. Lewis, and P. Sachsenmaier, Opt. Commun. 15, 231 (1975).

5. J. E. Bateman and M. H. Key, Rutherford Lab. (Oxford) Report No. RL-79-01 (unpublished, 1979) P. 17.

6. R. H. Day, P. Lee, E. B. Saloman, and D. I. Nagel, IEEE Conference Record-Abstracts, Conference on Plasma Science, Montreal, 1979 (IEEE, New York, 1979), p. 58.

7. P. A. Jáanimagi and M. C. Richardson, Bull. Am. Phys. Soc. $\underline{24}, 985$ (1979).

8. B. L. Henke, J. A. Smith, and D. T. Attwood, Appl. Phys. Lett. 29, 539 (1976).

9. B. L. Henke, J. Liesgang, and S. D. Smith, Phys. Rev. B19, 3004 (1979).

10. G. L. Stradling, H. Medecki, R. L. Kauffman, D. T. Attwood, and B. L. Henke, Appl. Phys. Lett. 37, 782 (1980).

11. B. L. Henke, J. P. Knauer, and K. Permeratna, J. Appl. Phys. 52, 1509 (1981).

12. E. L. Pierce, and D. T. Attwood, Lawrence Livermore National Laboratory report, "Laser Fusion Program Semi-Annual Report Ju1y-Dec. 1973", UCRL-50021-73-2, p. 56.

13. D. P. Gaines, G. L. Stradling, R. L. Kauffman, B. L. Henke, and R. T. Perkins, Bull. Amer. Phys. Soc. 25, 1148 (1980). 
Chapter 5

\author{
ELECTRON ENERGY TRANSPORT EXPERIMENTS \\ USING LAYERED- AND SOLID-DISK GEOMETRIES
}

\title{
Introduction
}

Implosion of laser-fusion targets is accomplished by the heating and rocket-like ablative expansion of material at, the surface of the spherical target. Most of the energy delivered to the target by moderate intensity 1.06-um laser light is converted, in the coronal plasma, to thermal-electron kinetic energy. ${ }^{1}$ These thermal electrons can deposit their energy in shallow surface regions of the target material. Experimental results have brought about considerable concern that energy transport by thermal electrons is inhibited below expected "classical" values. Inhibited energy transport results in less efficient target heating and, consequently, decreased target-implosion drive efficiency than otherwise expected. Extensive efforts to examine the problem of "inhibited" electron energy transport have been made both experimentally and theoretically. The work presented here is the first effort to examine thermal energy transport with time-resolved spectral measurements of thermal $x-r a y$ emission from a variety of relevant disk-target designs.

This chapter addresses the physics issues of thermal-electron energy transport, energy balance, and thermal x-ray spectral emission in laser-plasma interactions through high temporal resolution measurement of $x-r a y$ emission history from both layered- and solid-disk targets. The relationship of thermal $x$-ray emission to energy flow in the experimenis is discussed. The value and validity of the LASNEX ${ }^{2}$ numerical hydro-code simulation of the problem is emphasized. Comparisons are presented between time-resolved experimental data and detailed one-dimensional LASNEX simulations of specific experiments. Although the hydro-code simulations compare well with time-integrated measurements, temporal errors of $30 \%-50 \%$ are apparent in this first detailed comparison of time-resolved numerical simulations with time-resolved $x$-ray measurements. Such disparities point to the need for closer examination of physical processes in the laser plasma to improve our understanding of physics modeling of ICF target performance.

Organization of this chapter is as follows: the experimental Epproach and procedure are described followed by a brief summary of :te experimental results. A discussion of energy transport, physical wosting of inhibition mechanisms, and the LASNEX computer code then Ficis. A detailed discussion of the solid target and layered target expreimental results is then presented including comparisons with - imerzis simulations of the experiments. 


\section{The Experimental Approach}

Thermal $x$-rays are generated as material is heated to temperatures of order $100 \mathrm{eV}$. X-ray emission strongly increases as the temperature and electron density of the emitting region increase. Time-resolved measurements of thermal x-ray emission thereby trace the net temperature/density rise and fall in the (moving) emission region of the target.

Initially, the time-resolved spectral measurements were designed to monitor subkilovolt line emission from ionized oxygen and fluorine as a heat wave separately encountered these elements in consecutive layers of a multi-layered target. Such time-resolved measurements of sub-keV line emission, similar to experiments which have been conducted very successfully at higher, non-thermal photon energies ${ }^{3}$ ( $>1 \mathrm{keV})$, can provide detailed and unambiguous information on thermal-energy transport rates. Line emissions characteristic of specific ionized elements occur, of course, only when the elements are heated to appropriate temperatures. Continuum emissions, on the other hand, can result from electron-ion collisions between an energetic electron flux and cold background materials of any $z$. Measurement of

ionized lines instead of continuum emission verify that the emission region propagating into the layered target is indeed heated past some threshold temperature level.

Unfortunately, the Argus laser schedule and target fabrication constraints did not allow time for the intended shot series. Thus, thermal continuum emissions from simpler, solid- and layered-disk 
targets were time-resolved instead. Solid $\mathrm{Be}, \mathrm{Al}, \mathrm{Ti}$, and $\mathrm{Au}$ disks, as well as layered disks with thin Be layers of variable thickness on a 25- $\mu \mathrm{m}$ thick Al substrate (Fig. 5-1) were used as targets.

These simpler measurements do provide a useful mechanism for time-resolved checks of the thermal-energy transport models used in detailed hydrodynamic computer simulations of the experiments. The $x$-ray emission pulse shapes, each characteristic of a solid-disk target of a given $Z$ irradiated at one of several differont laser intensities, provide a check for comparison with the hydrocode's treatment of the balance of energy dissipation and energy transport factors.

Changes in the $\mathrm{x}-\mathrm{ray}$ emission pulse-shape were expected from layered targets of different overcoat thicknesses as the thermal-electron-driven heat front passed through the low-Z Be overcoat to the more strongly emitting Al substrate. An abrupt increase in $x$-ray emission would occur as a steep thermal gradient reaches the higher $-Z$ substrate. This emission step would be expected to shift later in time with respect to the intial Be emission on targets with thicker Be overcoats.

\section{The Experimental Configuration}

Figures 5-1 and 5-2 schematically show the configuration. Disk targets were irradiated by one beam of the Argus laser system. The disk targets were nominally $600-\mu \mathrm{m}$ in diameter and 25- $\mu \mathrm{m}$ thick. At 1.06- $\mu \mathrm{m}$ wavelength, energy on target was varied between three and ninety joules, and the laser spot diameter on target (converging beam) could be varied between $100 \mu \mathrm{m}$ and $440 \mu \mathrm{m}$ to provide desired irradiation intensities between $3 \times 10^{13} \mathrm{~W} / \mathrm{cm}^{2}$ and $10^{15} \mathrm{~W} / \mathrm{cm}^{2}$. Layered 


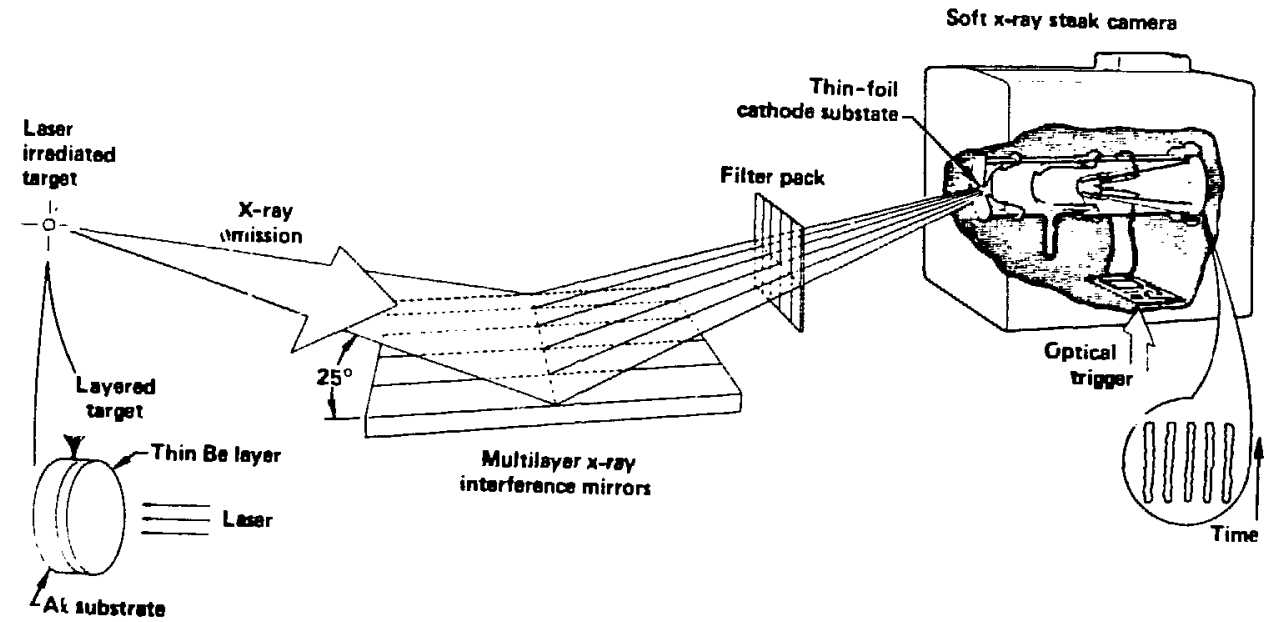

Fig. 5-1. The soft x-ray streak camera views $x$-ray emission from the heated target through a spectral discrimination apparatus. Here, five different, flat, metal-multilayer $x$-ray interference mirrors diffract narrow bands of the incident spectrum through thin transmission-filter foils and onto the streak-camera photocathode slit. A similar configuration is used for low-pass grazing-angle $x-r a y$ reflectors. These total-external reflection mirrors are used at a few-degrees grazing angles with absorption-edge transmission rilters to pass moderately-narrow spectral bands. The layered-disk target consists of a thin coating of $\mathrm{Be}$ on an $\mathrm{Al}$ substrate. 
targets were irradiated only at $3 \times 10^{14} \mathrm{~W} / \mathrm{cm}^{2}$ to moderate the hot component of the electron energy distribution in order to provide a more uniformly thermal electron distribution in the corona.

Intensity variations on target for any given shot were measured to be factors of two about the nominal intensity. Spot size and intensity variations in the target plane were determined using an equivalent plane camera external to the target chamber. In these experiments the laser temporal-pulse was Gaussian with a full-widthat-half-maximum (FWHM) of 700 psec $\pm 5 \%$ : however, most shots were monitored at the laser oscillator using an optical streak camera instead of at the target.

The disk targets were oriented with the surface normal at $0^{\circ}, 12^{\circ}$, or $30^{\circ}$ east of the south laser beam. The two $x$-ray streak cameras, one with broad energy channels, $E / \Delta E \sim 3-5, S X-1$ (soft $x$-ray streak camera \#1) and one with intermediate resolution spectral bins SX-6, viewed the face of the disk as shown in Fig. 5-2. Slower than maximum sweep rates were selected to allow large enough temporal windows to detect the full duration of the emission. In this case, temporal resolutions were degraded to numbers approaching 30 psec. Both detector configurations and spectral measurement windows are detailed in Chapter 3, Figs. 3-4, 3-5, 3-8, and 3-9. The intermediate spectral-resolution multilayer instrument (SX-6) viewed the target at $45^{\circ}$ to the east of the laser direction and $45^{\circ}$ below the horizontal plane. The broad-band resolution, reflector/filter instrument (SX-1) viewed the target from $90^{\circ}$ east of the incident beam and $22.5^{\circ}$ above the horizontal plane.

Pressure in the target chamber was maintained at $\sim 10^{-5}$ Torr. 


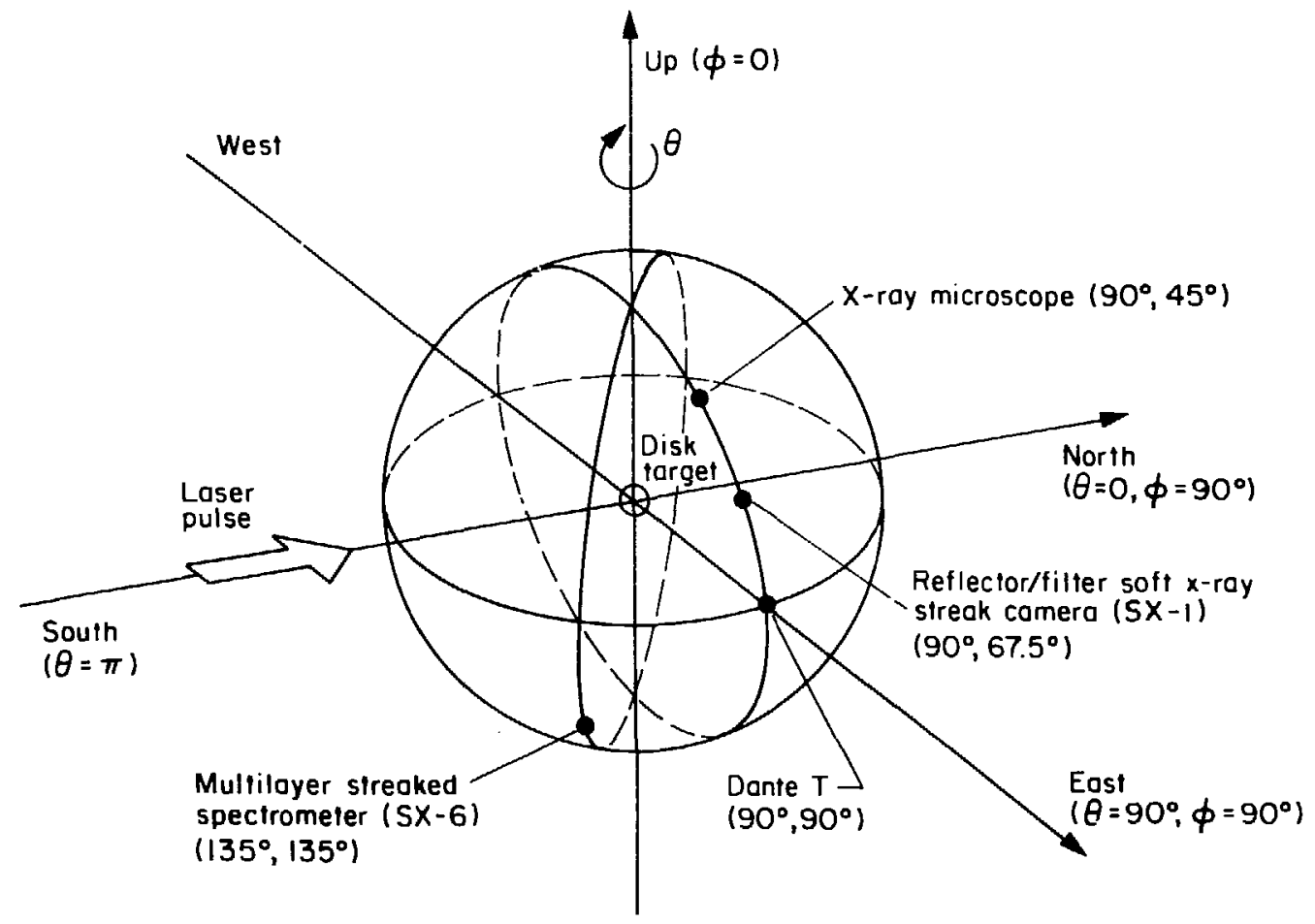

Fig. 5-2. Experimental geometry of disk target experiments at the Argus laser facility. The laser pulse is incident from the south. The target normal is positioned $0^{\circ}, 10^{\circ}$, or $30^{\circ}$ east of south. X-ray diagnostics viewing the target are the metal-multilayer $x$-ray-interferencemirror streaked spectrometer $(S X-6)$, the reflector/filter soft $x$-ray streak camera $(S X-1)$, an $x$-ray microscope, and filtered $x$-ray diodes (Dante). 
Summary of Results

I will briefly summarize the experimental results here with a detailed discussion of the experiment to follow later in the chapter.

Solid disk results. $X$-ray emission from solid single-element disks over a range of atomic Z's, and over a range of laser irradiation intensities, displayed variations in duration and temporal pulse-shape which depended strongly upon both parameters, $Z$ and $I_{\text {laser }}$ The laser temporal pulse-width was held constant. LASNEX simulations of these experiments also showed significant $x$-ray-emission temporal-shape variation with $Z$ and $I_{\text {laser }}$, but with quite a different functional dependence than observed in the measurements.

Spectral measurements from Au disks at the peak of the $x-r a y$ emission show a fairly flat subkeV spectrum. LASNEX closely matches this spectrum. Measurements are also presented showing peak $x-r a y$ emission-level variation with target $Z$ and laser intensity.

Layered disk results. The $x$-ray emission in several subkilovolt spectral bands from Be-on-Al layered targets exhibit smoothly rising and falling temporal shapes with a noticeable lack of distinct burn-through signature. Peak x-ray flux values decrease with increasing Be-coating thickness from the pure Al level to the pure Be level. While the LASNEx simulations agree reasonably well with this latter, more crude, measure of heat penetration into the target, the measured and calculated temporal shapes of the emission are markedly different from each other. One-dimensional numerical simulations of the problem predict an abrupt, stepped increase in the x-ray emission as a uniform heat wave zompletes passage through the Be overcoat and 
reaches the higher-Z Al substrate. The high-resolution temporal $x-r a y$ measurements, however, show no such abrupt burn-through to the Al substrate. Possible differences between the model used in the simulations and the phenomena of the experiment will be discussed later in the chapter.

\section{Energy Transport}

The rate of energy transport from the laser absorption region to the cold, dense target has a strong impact on the balance of energy distribution between plasma kinetic energy (PdV expansion), radiative dissipation, and target heating. The energy transport and dissipation mechanisms are illustrated in Fig. 5-3. Low transport rates for thermal energy logically result in a hotter plasma corona, and concomitantly reduced dense target heating and $x-r a y$ conversion efficiency. Deposited energy is then bottled up in the low-density corona and does not reach the dense region of the material where energy conversion to $x$ radiation is more efficient. Comparisons between earlier experiments and calculations show such symptoms of unexpectedly low energy transport. 1,4-9

Efforts have been made to match experimental results by modeling an electron "energy-transport inhibition," invoking a phenomenological limit to the free-streaming electron flux. ${ }^{10-15}$ This flux limiter is imposed when the classical Spitzer conductivity ${ }^{16}$ exceeds some fraction of the free-streaming limit. The justification, significance, size and necessity of such a flux limiter has been much debated in the literature. Some suggestions for lower-than-calculated electron mobility include scattering by strong megagauss microscopic 

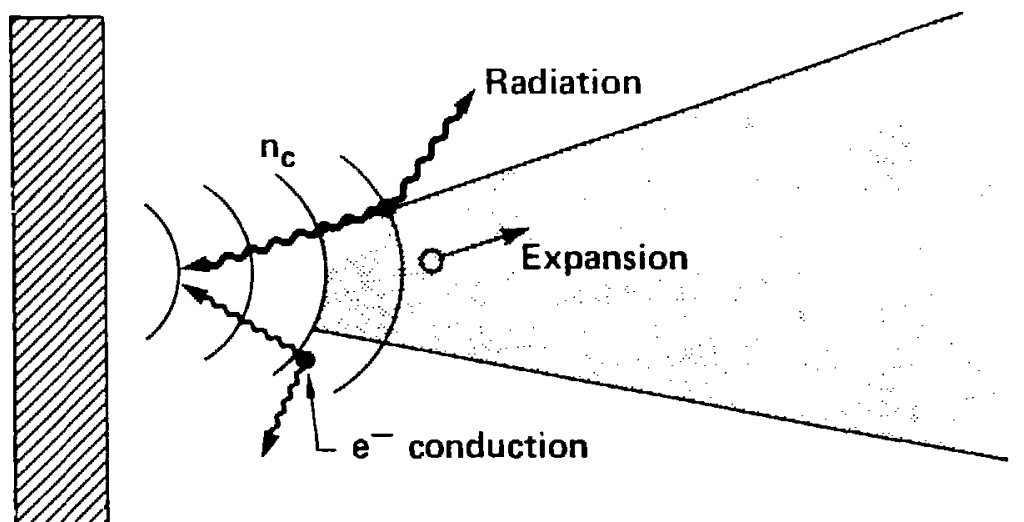

Laser beam

Cold dense matter

Fig. 5-3. Laser energy is deposited in the plasma up to critical

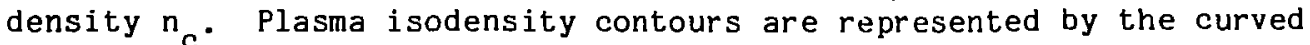
lines radiating out from the irradiation spot on the dense target. Energy deposited below critical density heats the underdense corona. Some of this energy is transported to denser regions, closer to the target, by thermal electrons, by more energetic electrons and by $x$ rays. The effectiveness of these processes depends upon the range of the energy carrier and the energy flux in either electrons or $x$ rays available from the corona. 
or macroscopic magnetic fields ${ }^{6,17-20}$ or electron scattering off of two-stream-instability induced ion-acoustic turbulerce. ${ }^{21-25}$ others suggest that a more careful treatment of classical transportcoefficients under steep density gradient conditions, ${ }^{26-30}$ or more detailed Monte Carlo treatment ${ }^{31}$ of classical electron transport will naturally account for the observed energy-transport rates.

Ion-acoustic electron transport inhibition has been observed in microwave simulations ${ }^{32}$ of laser-plasma experiments. The validity of scaling microwave experiments to the laser-plasma regime is not yet certain, however. Experimental evidence of ion-acoustic turbulence in 1.06- $\mu m$ laser-plasma experiments of interest is still elusive due to the short wavelength, but new work and suggestions have recently been made. 33.34 Ion-acoustic turbulence in 10.6- $\mathrm{m} \mathrm{CO}_{2}$ laser plasmas has been observed 35.36 using Thompson scattering. Such an effect should scale in wavelength to $1.06-\mu \mathrm{m}$ conditions. 37

Macroscopic, megagauss magnetic fields have been observed in the underdense region of 1.06- $\mu \mathrm{m}$ laser plasma experiments. 38 Such magnetic fields have not been detected in experiments probing close to critical density, where the laser absorption peaks in efficiency. 39,40 Explanations of the discrepancy between measured and calculated energy-trarisport phenomena is still not satisfactorily resolved.

\section{Interesting Experimental Designs}

Direct measurement of electron mean-free-paths under laser-plasma conditions is extremely difficult at best, and is in fact only recently being attempted. A quantity of interest, the energytransport rate, is somewhat more accessible. An indirect measure of 
the energy-transport rate may be had from the $x$-ray emission history of a simple, single-material target. Details of $x$-ray emission can provide a measure of plasma temperature. With a correct understanding of the physical processes, the PdV cocling rate and radiative cooling rate might be calculated. If, in addition, the laser energy deposition rate is known and the mechanisms for energy balance among the various processes are properly understood, the dense target heating rate might be self-consistently obtained. Distribution of absorbed energy among the items in the energy budget is shown schematically in Fig. 5-4.

A more direct measure of the energy transport rate may be obtained using distinct position and temperature dependent $x-r a y$ emitters to label the targec temperature as a function of time at positions of interest in the target. Such a target may be designed with thin layers of materials at various depths below the irradiation surface. Each material is selected for characteristic emission at unique and unambiguous $x$-ray wavelengths as it is heated to thermal temperatures. For temperatures of up to a few hundred electron volts, such dominant emission lines will lie below $\sim 1 \mathrm{keV}$.

Layered targets have been used by a number of groups to provide spatial diagnostic information at higher photon energies. Time integrated measurements have been made of layered target emissions by a number of research groups. $3,41-44$ Line emission at greater than $1 \mathrm{keV}$ photon energies have been time resolved at Rutherford. 3

The material temperature may be obtained from the ratio of atomic resonance lines 45 characteristic of ionized states. This layered, emission-line labeled target design provides a time-dependent 


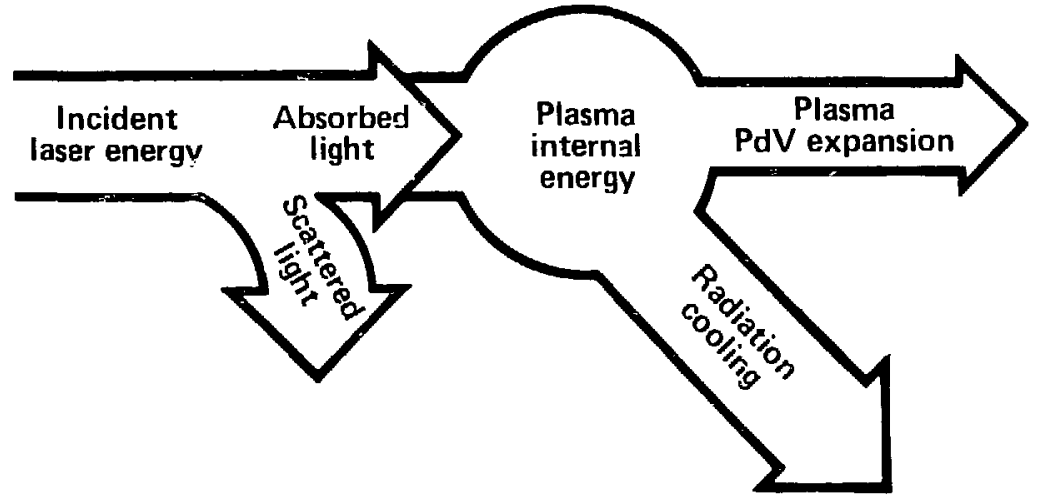

Fig. 5-4. The energy delivered to the target is budgeted into several distinct categories. A large fraction of the incident energy is scattered away from the target. The remainder, which is absorbed, heats and ionizes the target material. The energy used to produce this hot, ionized plasma is termed the internal energy of the plasma. The massive target serves as a heat sink eventually cooling the plasma. Heated electrons produced by laser-plasma interactions generate bremsstrahlung $x$ rays through close encounters with heavy nuclei. Atomic free-bound and bound-bound transitions also generate $x$ rays. $X$ rays which are radiated away from the plasma can provide another significant cooling mechanism. In addition, the plasma cools through expansion at the ambipolar potential, constrained at the sound speed $\mathrm{C}_{\mathrm{S}}$. 
signature of thermal-energy deposition in the target. High spectraland temporal-resolution diagnosis is required for this type of experiment.

An appropriate layered target design for thermal energy transport investigation consists of a thin $\mathrm{CH}$ layer over a thin Teflon (CF) layer, all coated onto a glass $\left(\mathrm{SiO}_{2}\right)$ substrate. Thermal-front propagation rates can be obtained from tempo:al delays between the turn-on times of the initial continuum emission from the $\mathrm{CH}$ overcoat, the $737-\mathrm{eV}$ helium-like fluorine emission line from the sandwiched Teflon layer, and the 653-eV hydrogen-like cxygen emission line from the glass substrate. These materials, the order of layers, and the specific emission lines are selected for visibility and uniqueness. Emission-line labels of the inner layers will not be obscured by earlier emission from outer layers.

Narrow-band (10-eV wide) spectral channels centered on the two characteristic emission lines of this target design as well as at 102-eV, 267-eV and 943-eV were implemented on the metal-multilayer soft $x$-ray streak camera. Unfortunately, the Argus laser shot schedule and target fabrication schedule did not ailow the time necessary to undertake this experiment.

Consequently, time-resolved measurements of subkeV continuum $x$-ray emission from simpler solid- and single-layered disk targets were performed instead. Temporal pulse-shapes of thermal-x-ray-emission from the solid, single-element, $\mathrm{Be}, \mathrm{Al}$, and $\mathrm{Au}$ disk targets provide integrated information on the energy balance and energy transport mechanisms operating in the laser plasma as discussed above. 
The layered targets consist of thin, variable-thickness, Be coatings deposited on a thick Al substrate (Fig. 1). The time required for energy to be transported through the Be overcoat and to generate thermal $x$-ray emission in the AI substrate may be monitored through the thermal x-ray emission pulse snape. Continuum $x$-ray emission levels from Be are significantly lower than from Al. Thus, an abrupt increase in emission is expected as a uniform, steep thermal gradient encounters the Al substrate. Timing between the initial $\mathrm{Be}$ emission and the abrupt Al-emission step would provide a measure of energy transport rates through different Be overcoat thicknesses. A steep, uniform thermal gradient is required, however.

Similar, but time-integrated, experiments $s^{41-44}$ have been performed in which the thickness of a layer of low-Z material on an $x-r a y$ emicting substrate is varied from shot to shot. Time-integrated $x-r a y$ emission (both thermal and high-energy) from the substrate is evaluated as a function of coating thickness. The energy transport rate is not directly accessible with this technique, although the range of possible electron mean-free paths may be constrained to match the experiment.

Energy transport experiments, temporally and spectrally resolving line emission $>1 \mathrm{keV}$ have been performed at Rutherford ${ }^{3}$ with considerable success.

The two categories of subkeV experiments reported here are the only temporally resolved, spectrally resolved measurements to date which specifically address thermal energy transport. Temporal-history measurement,s of solid, single-element disks of varying $Z$ 's over a range of laser intensities are discussed. Fully time-resolved 
measurements are also described of continum $x$-ray emission from targets with variable-thickness low-Z Be coatings of intermediate-Z Al substrates at a moderate laser intensity of $3 \times 1 u^{14} \mathrm{w}^{3} \mathrm{~cm}^{2}$. Soft x-ray streak cameras with both intermediate (E/AE - In to 90, SX-6)

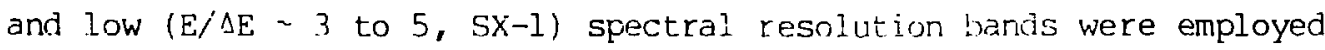
in the measurements.

\section{Analysis}

The electron energy distribution in the experiment is not known explicitly. However, electron temperature gradients are large near the critical density surface. Energy transport is mainly due to electron conduction into the target in these low-Z materials where $x$-ray emission in the corona is low and represents only a small fraction of the energy deposited above $n_{C^{*}}$ of course, the electron energy distribution is strongly dependent upon the rate of energy transport. Likewise the energy transport rate is governed by the energy-dependent electron mean-free path in the material.

Estimates of electron energy distributions are attempted through interpretation of the $\mathrm{x}$-ray emission spectrum. Such analysis is indirect and is further compromised by incomplete knowledge of subkeV $x$-ray spectral detail. Until recently, time-resolved spectral measurements were spatially integrated over the entire emission area of the target and consisted of measurements of a few discrete $x$-ray energy-bands rather than continuous speccra. Space and timeintegrated continuous spectra, averaged over the target and the duration of the emission, were also measured. Even within the 1 imits of these diagnostics, subkev spectral emission was seen to be 
non-Planckian. 46,47 As a recent continuation of this work with soft x-ray streak cameras, time-resolved spectrally dispersed subkeV data have been taken on this same series of target shots using $x$-ray transmission gratings. 48

X-ray measurements have also shown a significant high-energy component to the spectrum which is most pronounced at high laser irradiances. This effect is an indication of a supra-thermal electron distribution, in addition to the thermal electron distribution. The intent of these layered target transport experiments was to investigate thermal electron transport. Thus a moderate laser intensity of $3 \times 10^{14} \mathrm{~W} / \mathrm{cm}^{2}$ was used to reduce the long mean-freepath, high-energy eiectron contribution to energy transport in the problem.

X-ray emission from a disk target is determined from the interplay of time-dependent density $\rho$, temperature $T_{e}$, and ionization state variations $Z_{\text {eff }}$ through the plasma and into the target. SubkeV $x-r a y$ emission at a given depth, $z$, in the target and at a given time may be obtained from the bremsstrahlung, free-bound, and bound-bound emission cross section for the energy distribution of the energy transporting electrons. The dependence of the bremsstrahlung spectral emission rate $\mathrm{J}_{\mathrm{v}}$ on plasma parameters is given by: ${ }^{49}$

$$
J_{v} d v=\frac{32 \pi}{3}\left(\frac{2 \pi}{3 k T e_{e}{ }_{e}}\right)^{1 / 2} \frac{z^{2} e^{6}}{m_{e} e^{3}} n_{i} n_{e} \exp \left(-\frac{h v}{k T_{e}}\right) d v
$$

Here hv is the emitted photon energy, e is the electronic charge, $c$ is the speed of light in a vacuum, and $n_{i}$ and $n_{e}$ are the number density of ions and electrons. 
The free-bound spectral emission rate is given by:

$$
J_{v} d v=1.7 \times 10^{-40} n_{i} n_{e}\left(\frac{I_{H}}{k T_{e}}\right)^{3 / 2}
$$

$$
\times \sum_{n} \frac{z^{4}}{n^{5}} 5_{n} B_{b f} \exp \left[\frac{-n v+\frac{I_{H} z^{2}}{n^{2}}}{k T e}\right] \frac{\operatorname{erg}}{c c}
$$

for all $n$ such thet $\frac{I_{H} z^{2}}{n^{2}}<h_{\nu}$.

The number of positions available in the $n^{\text {th }}$ ionization state is $\zeta_{n}$ and $B_{b f}$ is the bound-free gaunt factor. $I_{H}=2 \pi_{i}^{2} m_{e} e^{4} / h^{2}$ is the binding energ; nf the hydrogen atom.

Bound-bound emission is rery much more difficult to calculate. A photon with energy $h v_{i j}$ corresponding to a transition between levels $i$ and $j$ in the partially ionized atom in the $i^{\text {th }}$ state has the emission probability $A_{i j} \cdot A_{i j}$ is the Einstein coefficient for emission.

The energy emitted in line hu ${ }_{i j}$ per unit volume per unit time is then $n_{i} A_{i j} h v_{i j}$ where $n_{i}$ is the atomic number density in the $i^{\text {th }}$ excited state. 51 Rate equations determine $n_{i}$ in the context of all other states in the plasma as well as the plasma density and temperature.

These processes each depend upon the density, temperature, and ionization state of the material. These plasma parameters and the electron energy distribution $f_{e}(E, z)$ depend, in turn, on the plasma conditions at shallower depths. 
The $x$-ray transmission from the emission region of the plasma to the detector is, in turn, dependent upon the $x$-ray absorption cross section and material density along the transmission path. X-ray emission $F_{x}$ from a disk target at a given time may be modeled as;

$$
F_{x}=\int_{0}^{\infty} d z \int_{0}^{\infty} d E \frac{d E}{d z} n_{x}\left(\exp -\left[\int_{0}^{z} \mu \rho d l\right]\right) f_{e}(E, z)
$$

The energy deposited per differential depth, dE/dz, (integrated over all electrons in the energy distribution $f_{e}(E, z)$ at position $\left.z\right)$ is converted to $x$ rays with a conversion efficiency $n_{x}$ which depends on the $x$-ray production relations given above. This radiation is transmitted to the surface through an average absorption-coefficient/ density product, $\int_{0}^{z} \mu \rho d l$.

Because of the complicated temperatume, density, ionization state, position, and time dependence of the factors in equation $5.3, F_{x}$ cannot be solved in closed form. Meaningful evaluation of $F_{x}$ requires a full-scale calculation using the detailed $\rho, T_{e}, f_{e}$ parameter variations obtained in numerical simulations of the target hydrodynamics and plasma physics.

\section{LASNEX Simulations}

W. C. Mead at LLNL has performed the detailed one-dimensional computational simulations of the experiments of interest. These calculations were designed to simulate the experiments in accordance with state of the art understanding of the relevant physical processes 
and our best knowledge of experimental parameters. LASNEX follows the basic material parameters on a spatial grid in finile temporal steps. For example, calculated spatial dependence of the basic plasma parameters at the peak of the laser pulse in a pure Be target are shown in Fig. (5-5). Also plotted in this figure are the position-dependent contributions to the overall $x$-ray emission.

The LASNEX simulations used here are one-dimensional, Lagrangian, hydrodynamic calculations. Energy transport is approximated by multi-group, flux-limited diffusion of electrons and multi-group propagation of $x$ rays. The electron energy flux $Q_{e}$ is limited by an electron flux inhibitor $f$ of .01 for both thermal and supra-thermal electrons. $Q_{e}$ is determined by the code as:

$$
Q_{e}=\min \left[-k \nabla T e, f n_{e} e^{-1 / 2}\left(k T e^{3 / 2}\right]\right.
$$

where $k$ is the Spitzer conductivity, $f$ is the flux inhibitor, $n_{e}$ and $m_{e}$ are the slectron density and mass and $k \mathrm{~T}_{e}$ is the electron temperature in energy units. Plasma physics processes such as laser absorption and scattering along the density profile and the distribution of absorbed energy into thermal and supra-thermal electron-energy distributions are handled by the code with ad hoc recipes. The recipes for treating these processes are formulated from empirical, experimental and calculational, results as well as from plasma physics theory. X-ray emission and absorption are handled with average-atom atomic physics approximations. When necessary, a non-LTE atomic physics package is used to calculate the rate equations. 

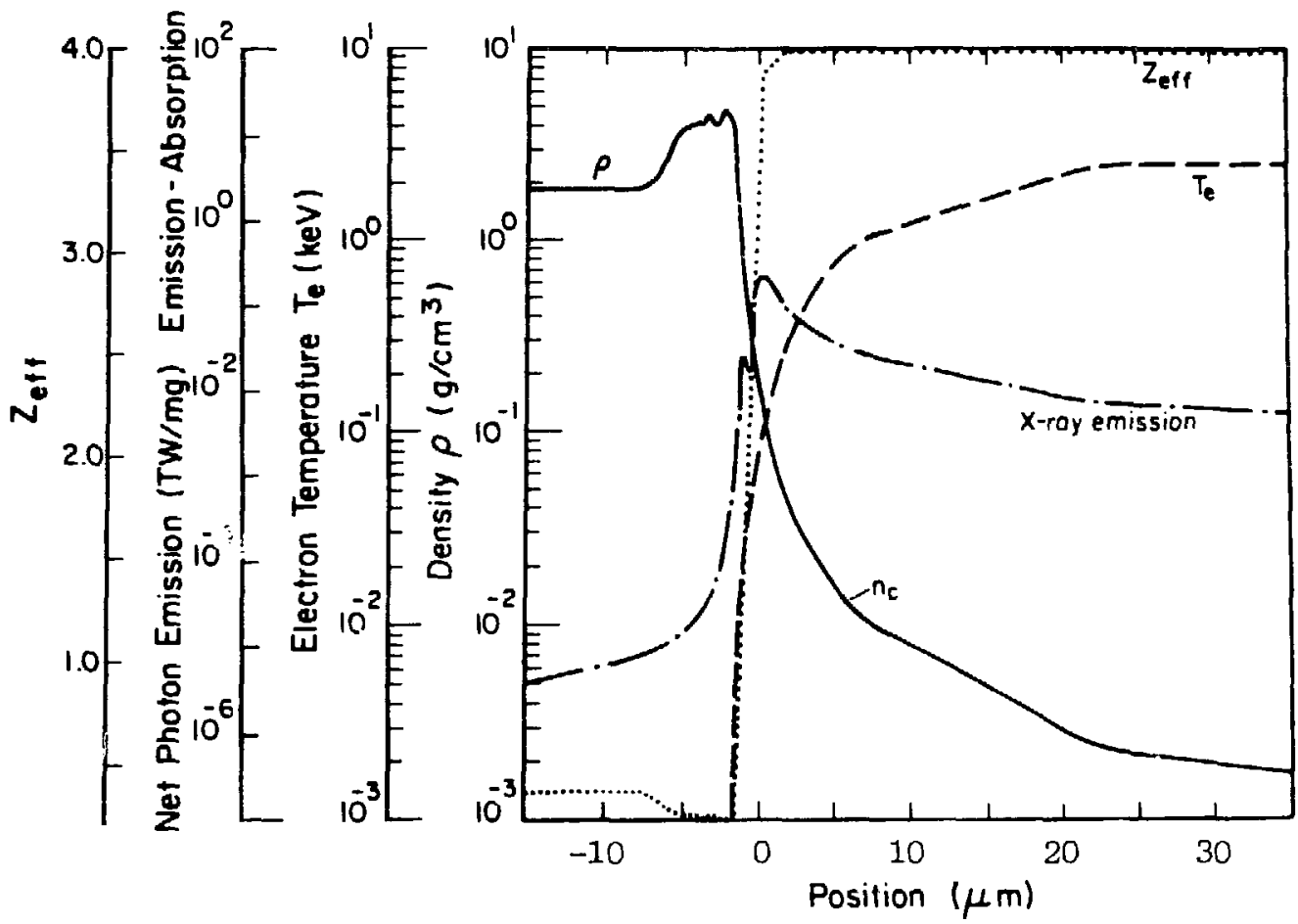

Fig. 5-5. Calculated spatial profiles of the fundamental Be plasma parameters: density $\rho$, electron temperature $T$, and ionization state $Z_{\text {eff }}$ as well as the spectrally integrated $\mathrm{x}$-ray-emission source distribution. The profiles are calculated in one dimension at the peak of the laser pulse. The axial-position axis is common to all of the profiles with the disk face position at 0 um. Amplitude scales are specified separately for each parameter. The plasma density rises to critical density $n_{c}$ about $-8 \mu m$ in front of the disk face. A compression wave is generated behind the disk surface. The electron temperature in the corona is $2.5 \mathrm{keV}$, falling to $800 \mathrm{eV}$ at $\mathrm{n}$ and to $100 \mathrm{eV}$ at the peak $\mathrm{x}$-ray emission point. X-ray emission is

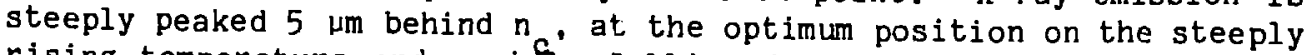
rising temperature and rapidiy falling density profiles. The $\mathrm{Be}$ is stripped of all electrons over a narrow 2- $\mu$ m transition region. 
Energy Transport in Solid Disks as a Function of Target $Z$

Temporal $x$-ray-emission signatures were measured for a variety of elements ( $Z^{\prime} s$ ) and over a range of laser-irradiation intensity levels. The measured emission pulse-shapes are found to be characteristic of the experimental parameters and aro repeatable.

\section{Experimental Conditions}

Disk targets, solid $B e, A l$, and $A u$, oriented with the surface normal $30^{\circ}$ east of the laser direction, were irradiated with 700-psec \pm 5\% FWHM, 1.06- $\mu \mathrm{m}$ laser pulses. Irradiation intensity levels were varied between $3 \times 10^{13} \mathrm{~W} / \mathrm{cm}^{2}, 3 \times 10^{14} \mathrm{~W} / \mathrm{cm}^{2}$, and $1 \times 10^{15} \mathrm{~W} / \mathrm{cm}^{2}$ by selecting appropriate combinations of laser energy and target spot size. Laser energy was varied between $3 \mathrm{~J}$ and $90 \mathrm{~J}$. Irradiation spot size diameters on the target were varied between $85 \mu \mathrm{m}$ and $420 \mu \mathrm{m}$. Intensity variations across the irradiation spot, in these well controlled low power laser shots, were typically factors of two. 52

LASNEX simulations were one dimensional, with a flux limiter $f$ of 0.01 , 600-psec FWHM Gaussian laser pulse-shape and witil a full non-LTE atomic physics package. Temporal results from the calculations shown here have been dilated in time by $7 / 6$ to match the experimental pulse-length because 700-psec calculations have not been performed. This correction should approximately scale processes which are short on the laser time scale. However, radiative bulk cooling, when it extends appreciably beyond the laser pulse decay, will be scaled longer than the corresponding physical phenomena. 


\section{Temporal-Emission Pulse-Shape Comparisons}

The temporal soft $x$-ray emission from disk targets may be considered to nave the shape of the energy-deposition pulse, modifiei by extended cooling. Cooling of the target by a combination of radiation and plasma expansion on time scales longer than the laser pulse-length will extend the $x$-ray emission pulse-length. Radiation cooling rates depend on the target $z$ and temperature. The $x$-ray emission pulse-shape is also a function of detected photon energy. High-energy $(\sim 1 \mathrm{keV})$ emission tends to closely follow the laser pulse-shape for these pulse durations. Lower photon-energy emission continues for longer times, consistent with semi-equilibrium cooling of the emitter. Au data $\left(3 \times 10^{14} \mathrm{~W} / \mathrm{cm}^{2}\right)$ at $100 \mathrm{eV}$ shows emission pulse-lengths 1.7 times longer than the 700-psec laser pulse whereas 953-eV emission times are only $\sim 4 \%$ larger. 53

The energy-deposition pulse-shape may also be skewed from the laser pulse-shape by temporal dependence in energy transport rates 54 or by temporal variation in the laser absorption. Direct observation of energy-transport rate variations is, of course, a step more difficult than measurement of a uniform transport rate, of which this Dissertation is an early attempt.

Inefficient energy transport in the target, on a time scale short with respect to the laser pulse, results in a shallower, hotter plasma region than more efficient energy transport would produce (Fig. 5-3). Inefficient energy transport may be due to inadequately small quantities of electrons to carry the energy and/or because of efficient electron-scattering mechanisms in the plasma (such as ion-acoustic turbulence or strong magnetic fields). The hotter, 
smaller plasma which results may be expected to radiate its energy more efficiently, thereby cooling faster. Thus the $x-r a y$ emission

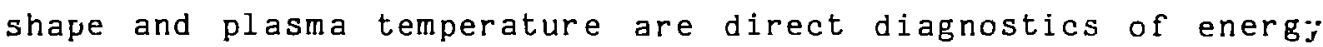
transport rates.

Maasurements of $x$-ray emission from Au disks at $3 \times 10^{13} \mathrm{~W} / \mathrm{cm}^{2}$, $3 \times 10^{14} \mathrm{~W} / \mathrm{cm}^{2}$, and $1 \times 10^{15} \mathrm{w} / \mathrm{cm}^{2}$, as well as from both Be and $\mathrm{Al}$ disks at $3 \times 10^{14} \mathrm{~W} / \mathrm{cm}^{2}$, are compared with LASNEX simulations of the $x$-ray emissicil pulse shapes in Figs. 5-6 through 5-10. In these linearly scaled pulse-shape comparisons, the calculated pulses are scaled in amplitude as well as positioned in time to inatch peak positions with the measurements. Both the 200-eV (carbon filtered) channel and the 700-eV (iron filtered) channel of SX-1 are shown. (Relative intensity units in the graphs of inese two channels are relative intensity units of the instrument, and are not adjusted to give either aisolute flux levels or relative flux levels between tre two channels.) The LASNEX calculations of these emissions include spectral filters chosen to match the channel spectral shapes of the reflector-filter soft x-ray streak camera.

Au disk pulse-shapes at three laser intensities. In bot.h data sets the lower-energy 200-eV channel shows longer emission times than the higher-energy $700-\mathrm{eV}$ channel. This is consistent with the expected semi-equilibrium cooling of the plasma. Measurements of $\mathrm{Au}$ at $3 \times 10^{13} \mathrm{~W} / \mathrm{cm}^{2}$ (Fig. 5-6) show a rapid Gaussian rise of the emission closely following the Gaussian, 700-psec FWHM laser pulse. Enission from $\mathrm{Au}$ at higher intensities (Figs. 5-7 and 5-8) show a slight amount of temporal structure and a perseptibly slower rise of the leading edge. The calculations seem to exaggerate the latter 
effect. While the fall-off in che x-ray pulse is similar in shape for both the measurements and the calculations of $\mathrm{Au}$ at the three irradiation intensities (Figs. 5-6, 5-7, and 5-8), the calculations give significantly longer pulse-widths. Only the 700-eV channel of the $1 \times 10^{15} \mathrm{~W} / \mathrm{cm}^{2}$ calculation (Fig. 5-8) is short enough to match the measurement. Higher intensities produce distinctly longer emission times in measurements at both channel energies.

Pulse-shape differences from disks of varying Z's. The lower-Z targets (Figs. 5-9 and 5-10) exhibit much more structure at the peak and on the tail of the emission pulse than does Au in the $3 \times 10^{14} \mathrm{~W} / \mathrm{cm}^{2}$ measurements. This temporal roughness can be separated from the effect of lower signal-to-noise ratio which occurs at reduced flux levels.

At $3 \times 10^{14} \mathrm{~W} / \mathrm{cm}^{2}$ the emission time (FWHM) of the lower- $z$ plasmas (Figs. 5-9 and 5-10) is significantly longer than that of Au (Fig. 5-6). The rise time is much like the Au data but the peaks are broader and the tail falls more gradually. More efficient heating of larger quantities of material may account for the difference between the low- and high-z emi ons. This could be a demonstration a longer cooling time from the less efficient, low-Z radiators, although their proportionally lower mass will also allow more efficient PdV expansion cooling.

The energy which is tied up in the kinetic motion of the plasma, the energy spent on stripping ions, and the energy radiated out of the plasma are all of nearly the same order of magnitude. 55 Each of these items in the energy budget affect the others through the plasma temperature, density and charge state as discussed earlier. 

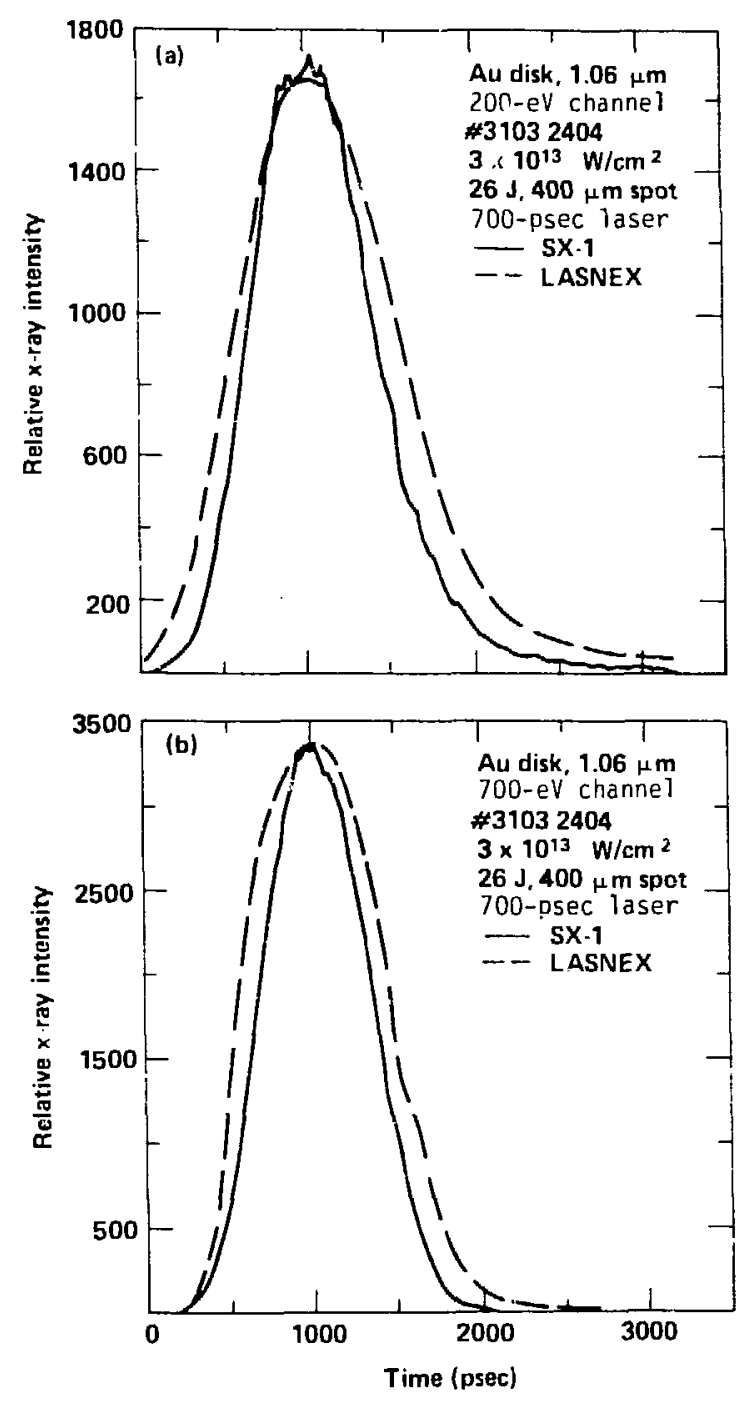

Fig. 5-6. Measured ard calnulated $x$-ray emission temporal pulseshapes from an $\mathrm{Au}$ disk irradiated at ? $\mathrm{x} 10^{13} \mathrm{~W} / \mathrm{cm}^{2}$ laser intensity. Parts $a$ and $b$ are emission in the $200 \mathrm{eV}$ and $700 \mathrm{eV}$ reflector/filter channels respectively. The LASNEX calculations in this case use spectral filter functions which match the spectral windows of the instrument. The Gaussian laser pulse-length was 700-psec FWHM. The LASNEX profiles are calculated from a 600-psec FWHM Gaussian Fuise-length but have been dilated in time by $7 / 6$ to compara wi $n$ the measurements. The calculations show a longer emission time cuan the measurements in both energy bands. 

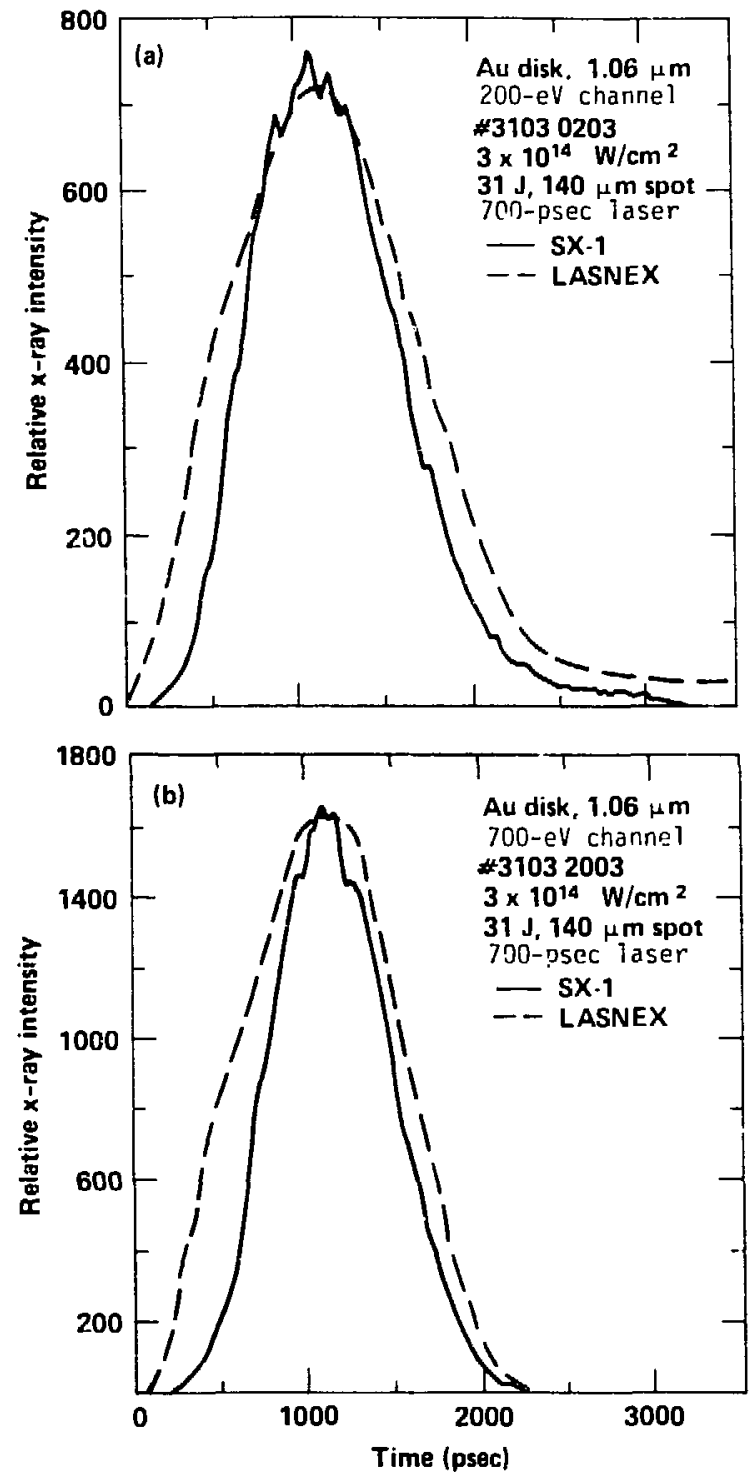

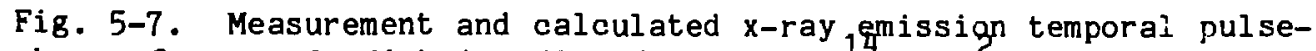
shapes from an Au disk irradiated at $3 \times 10^{14} \mathrm{~W} / \mathrm{cm}^{2}$ laser intensity. Parts $a$ and $b$ are emission in the $200-\mathrm{eV}$ and $700-\mathrm{eV}$ reflector/filter channels respectively. The LASNEX calculations use spectral filter functions which match the spectral windows of the instrument. The Gaussian laser pulse-length was 700-psec FWHM. The LASNEX profiles are calculated from a 600-psec FWHM Gaussian pulse-length but have been dilated in time by $7 / 6$ to compare with the measurements. The calculations show a much more gradual rise and longer emission time than the measured pulses. 

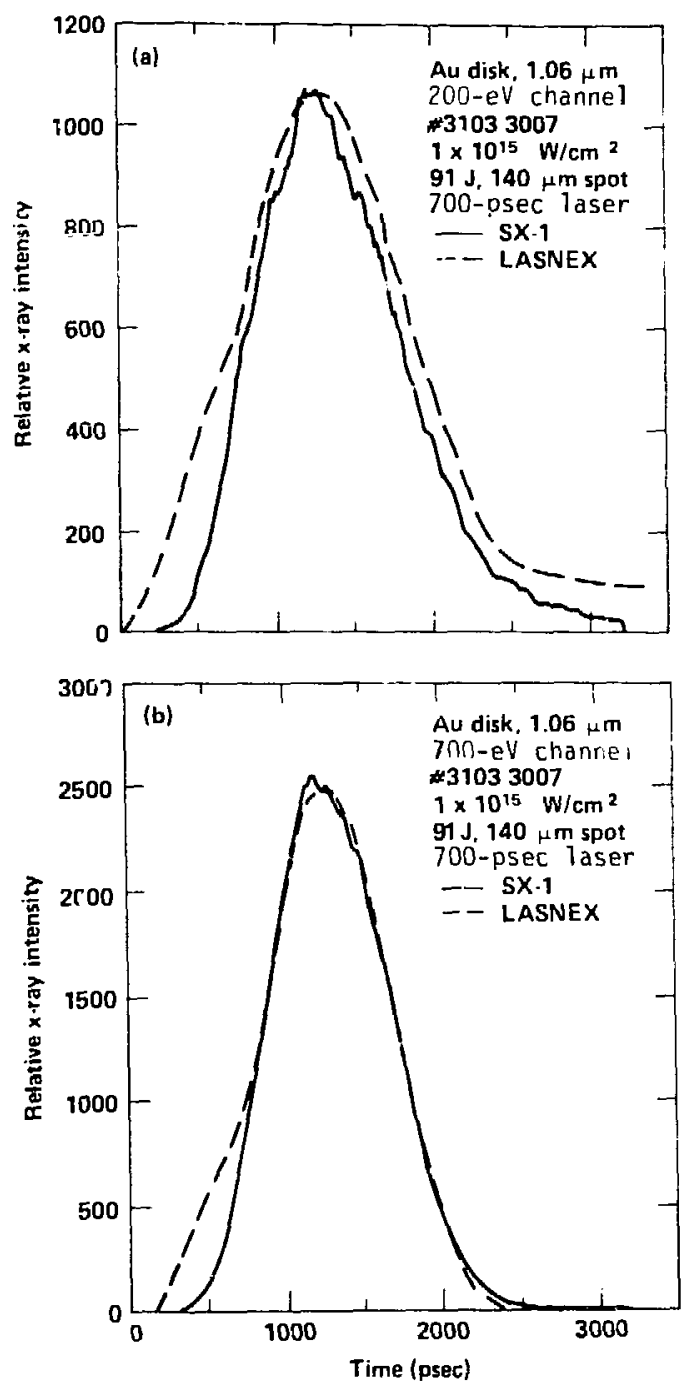

Fig. 5-8. Measured and calculated $x$-ray fmission temporal pulseshapes from an $\mathrm{Au}$ disk irradiated at $1 \times 10^{15} \mathrm{~W} / \mathrm{cm}^{2}$ laser intensity. Parts $a$ and $b$ are emission in the $200-\mathrm{eV}$ and $700-\mathrm{eV}$ reflector/filter channels respectively. The LASNEX calculations use spectral filter functions which match the spectral windows of the instrument. The Gaussian laser pulse-length was 700-psec FWHM. The LASNEX profiles are calsulated from a 600-psec FWHM Gaussian pulse-length but have been dilated in time by $7 / 6$ to compare with the measurements. The match between the calculated and measured pulse-shapes is very close except for a slightly more gradual rise at the toe of the simulated pulse. 

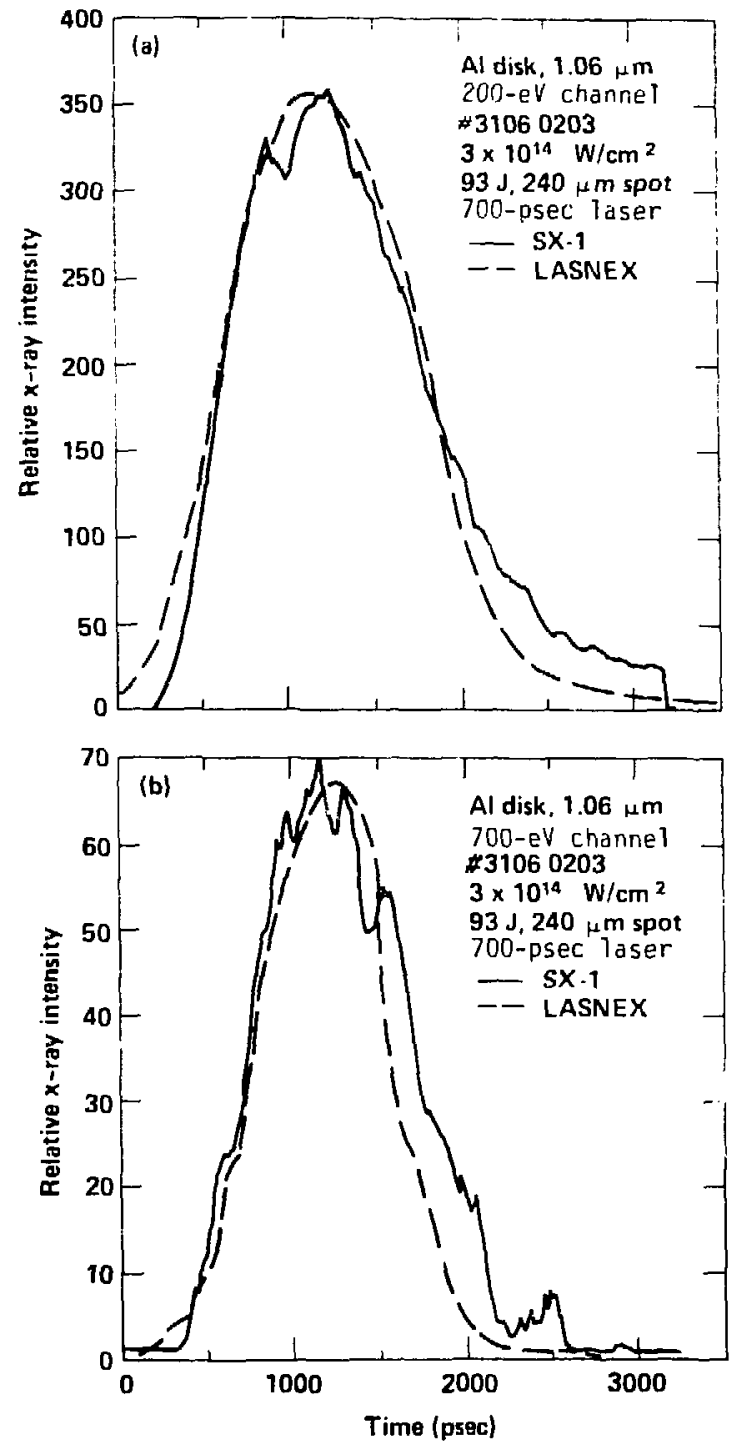

Fig. 5-9. Measured and calculated $x$-ray exissio; temporal pulseshapes from an Al disk irradiated at $3 \times 10^{14} \mathrm{~W} / \mathrm{cm}^{2}$ laser intensity. Parts $a$ and $b$ are emission in the $200-\mathrm{eV}$ and $700-\mathrm{eV}$ reflector/filter channels respectively. The LASNEX calculations use spectral filter functions which match the spectral windows of the instrunent. The Gaussian laser pulse-length was 700-psec FWHM. The LASNEX profiles are calculated from a 600-psec FWHM Gaussian pulse-length but have been dilated in time by $7 / 6$ to compare with the measurements. The low-energy channel is quite similar in both the measurement and the calculation. LASNEX predicts a shorter emission time than was observed in the $700-\mathrm{eV}$ channel. The $\mathrm{x}$-ray emission was consistently temporally rough in contrast to Au-emission temporal shapes. 


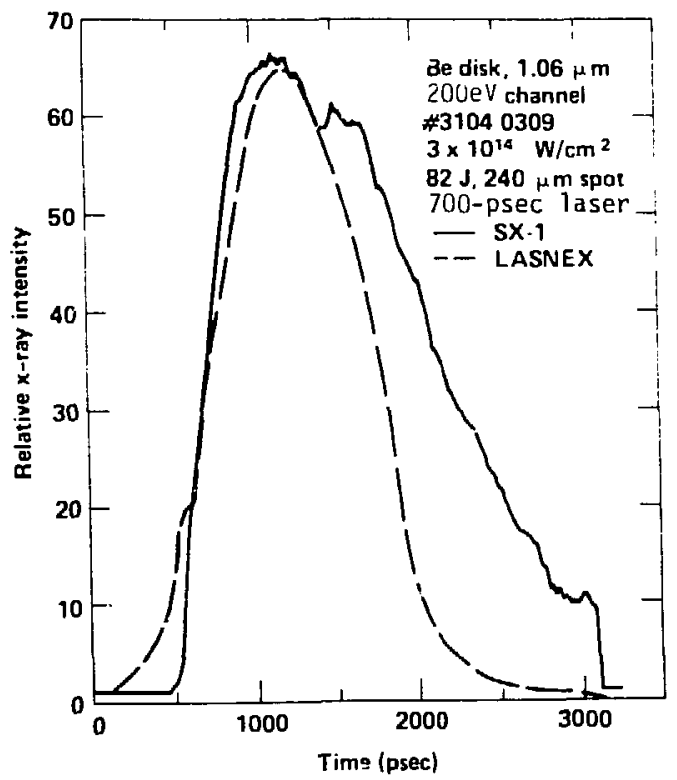

Fig. 5-10. Measured and calculated x-ray emission temporal pulseshapes from a Be disk irradiated at $3 \times 10^{14} \mathrm{~W} / \mathrm{cm}^{2}$ laser intensity. A spectral filter function was used in LASNEX to match the 200-eV channel spectral window. The Gaussian laser pulse-length was 700 psec FWHM. The LASNEX profile is calculated from a 600-psec FWHM Gaussian pulse-length but has been dilated in time by $7 / 6$ to compare with measurements. The emission time at the half-maximum point is much longer than predicted by LASNEX. Temporal structure on the peak of the $x$-ray emiseion was observed on every shot. 
The $200-\mathrm{eV}$ calculational pulse-shape from Al at $3 \times 10^{14} \mathrm{~W} / \mathrm{cm}^{2}$ (Fiz. 5-9a) closely matches the overall shape of the measured emission. The 700-eV calculation (Fig. 5-9b) has a significantly shorter pulse-width than the measurement. The calculation of Be emission pulse-shape at $3 \times 10^{14} \mathrm{~W} / \mathrm{cm}^{2}$ into the 200-eV (carbon filtered) channel is a great deal narrower than the measuremert (Fig. 5-10). There are important deficiencies in the modeling clearly apparent in these comparisons.

\section{Summaries of Pulse-Width Dependencies on Laser Intensity and Target $Z$}

Figures 5-11 and 5-12 summarizes the pulse-width trends from both the measured and calculated $x$-ray emission pulse-shapes in both the 200-eV (carbon filtered) channel and the 700-eV (iron filtered) channel. The ratio of $x$-ray emission pulse-width (FWHM) ${ }^{\tau} x$ to the laser pulse width (FWHM) ${ }^{\top}$ laser is taken from the Au disk data (Figs. $5-6,5-7$, and 5-8) and plotted as a function of laser intensity in Fig. 5-11. Similarly, the ratio of $\tau_{x} /{ }^{\top}$ laser from the data of $3 x$ $10^{14} \mathrm{~W} / \mathrm{cm}^{2}$ irradiations of several disks of different Z's (Figs. 5-7. 5-9, and 5-10) are plotted in Fig. 5-12.

These summaries reduce the variations in the shapes of the leading edge, peak, and tail of the emission pulses to a single pulse-width parameter. However, clear trends in emission time variations with the two parameters ( $\mathrm{I}_{\text {laser }}$ and $\mathrm{Z}$ ), and distinct differences between the measurement trends and calculational trends, justify the simplification. 


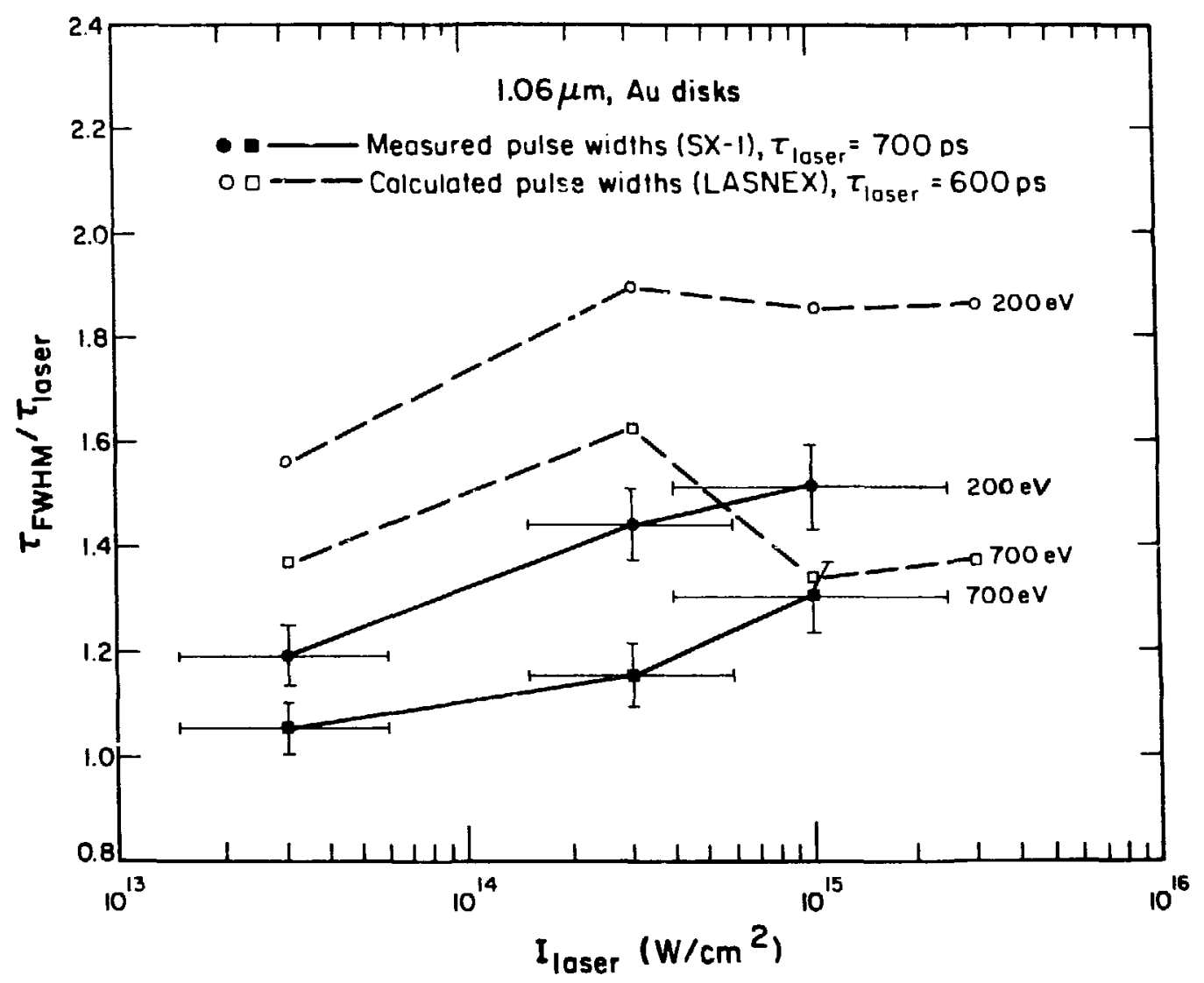

Fig. 5-11. The ratio of $\mathrm{Au} x$-ray emission pulse-widths to the laser pulse-width as a function of laser intensity. Observed pulse-widths are compared with those predicted by LASNEX simulations. This figure summarizes the gross features of Figs. 5-6 through 5-8. LASNEX consistently predicts emission times significantly longer than ouserved, by a factor of 1.3 to 1.5 . 


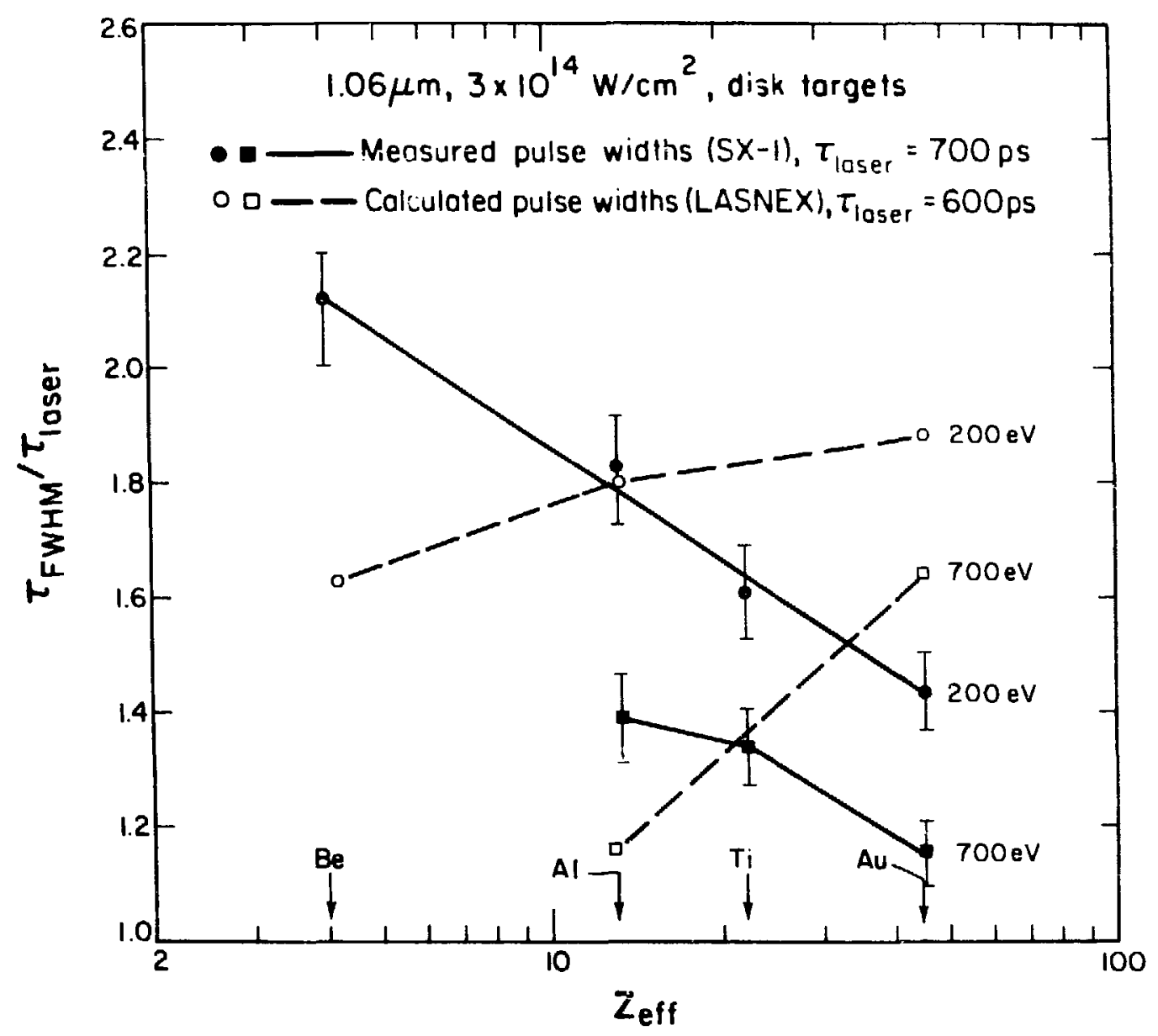

Fig. 5-12. X-ray emission pulse-widths, normalized to the laser pulse-width, as a function of target $Z$. Measured pulse-widths display a different trend in $Z$ dependence to those predicted by LASNEX simuiations of the experiments. This figure summarizes the gross features of Figs. 5-7, 5-9, and 5-10. LASNEX consistentiy predicts emission times significantly, fonger than measured. Both the measurements and simulations usc $3 \times 10^{14} \mathrm{~W} / \mathrm{cm}^{2}$ laser intensity. 
Summary of the variation with intensity of Au temporal pulsewidths: In Fig. 5-11 the measured-emission pulse-width is seen to increase gradually with laser intensity at both channel energies. The vertical error bars on the measured-data points represent uncertainty in the soft $x$-ray streak camera sweep-rate as well as in the laser pulse-width. The calculated pulse-shapes are significantly broader out exhibit a similar trend as the measurement at $3 \times 10^{13} \mathrm{~W} / \mathrm{cm}^{2}$ and at $3 \times 10^{14} \mathrm{~W} / \mathrm{cm}^{2}$ in both energy channels. This extra broadening in the calculation has contributions both from the calculated emission pulse rise and from the pulse decay as is evident in Figs. 5-6 and 5-7. The slight drop in pulse-width of the calculated 200-eV channel at $1 \times 10^{15} \mathrm{~W} / \mathrm{cm}^{2}$ is not inconsistent with the uncertainties in the measured-data trend between $3 \times 10^{14} \mathrm{~W} / \mathrm{cm}^{2}$ and $1 \times 10^{15} \mathrm{~W} / \mathrm{cm}^{2}$. However, the calculated 700-eV pulse-width shortened dramatically from the $3 \times 10^{14} \mathrm{~W} / \mathrm{cm}^{2}$ value to closely match the measured pulse-shape. Calculated, $3 \times 10^{15} \mathrm{~W}^{\prime} \mathrm{cm}^{2}$ pulse-shapes are not shown but the pulse-widths continue the trends as plotted.

The code physics has been adjusted to account for observables which, up to this time, have had only modest temporal and spectral resolution. With the presentation of new data which has higher temporal and spectral resolution, it is clear that further adjustments to the code physics must be made.

Summary of temporal pulse-width variations with target $z$. The emission pulse-width of laser pulse-width ratio, $\tau_{x} / \tau$ laser , is plotted as a function of $Z$ in Fig. 5-12 for the Be, Al, and $A u$ pulse-shape data taken at $3 \times 10^{14} \mathrm{~W} / \mathrm{cm}^{2}$ (Figs. 5-7, 5-9, and 5-10). Again, such a simplification is a reasonable means of analyzing these regular 
pulse shapes. The measured $\mathrm{Ti}$ data points included here have no corresponding pulse-shape calculations for comparison. Again the vertical error bars represeri uncertainties in the streak camera sweep-rate and the laser pulse-width.

A distinct trend of shorter emission times from higher-Z targets is apparent in the measurements in both energy channels. The 200-eV Be measurement is 50\% longer than the 200-eV Au emission puls?.

This effect may be cue to the increased radiative efficiency of the higher- $Z$ materials more than compensating for the reduced expansion-cooling rate of heavier ions. Another possibility is that radial energy transport may be more efficient in lower-z materials, resulting in a larger, cooler emission region and, thus, slower cooling.

Data showing the nearly linear increase in peak enission levels with increasing target $z_{\text {eff }}$ (Fig. 5-13) demonstrates the level of emission efficiency as a function of $Z$. These data are from $3 x$ $10^{14} \mathrm{~W} / \mathrm{cm}^{2}, 20 \mathrm{j}$ irradiations of disk targets. Ionization state $\mathrm{Z}$ eff ranges are taken from LASNEX calculations.

The calculated pulse-widths, plotted with the measurements in Fig. 5-12, tend to increase with increasing target $Z$. This trend is opposite to that observed in the measurements. The calculation is $225 \%$ low at the $200-\mathrm{eV}$ be point and $-30 \%$ high at the $200-\mathrm{eV}$ and $700-\mathrm{eV}$ $\mathrm{Au}$ points. Again these differences represent significant errors in temporal energy distribution in LASNEX and merit further effort to isolate where the differences arise, through use of more detailed multilayer target experiments. 


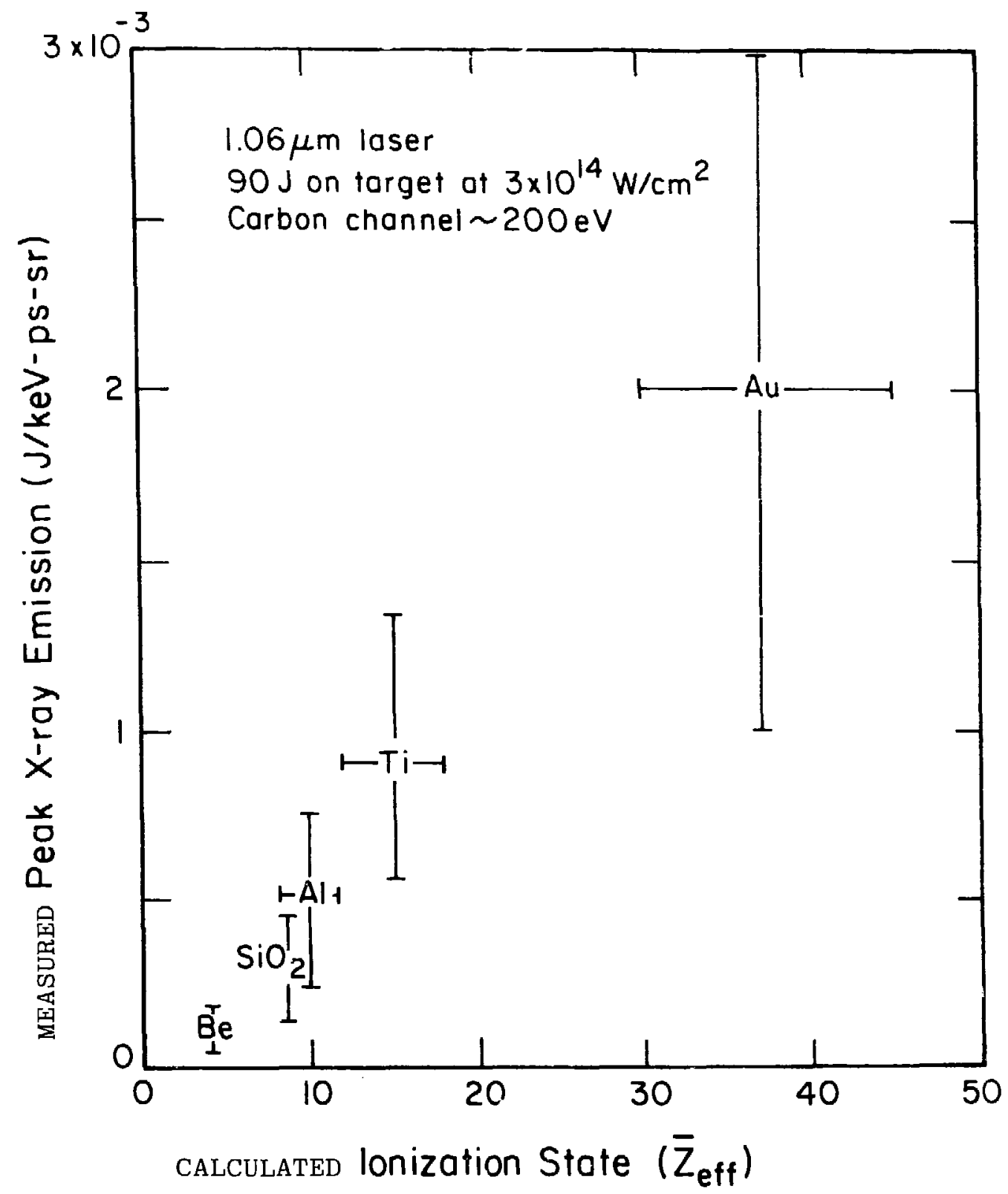

Fig. 5-13. The peak $x$-ray emission level in the 200-eV carbon channel from $S X-1$ is seen to vary nearly linearly with the $Z_{\text {eff }}$ of the emitting plasma. 
Spectral Data

Measured peak $x$-ray emission in several spectral channels from Au disks irradiated at $3 \times 10^{13} \mathrm{~W}^{\prime} \mathrm{cm}^{2}, 3 \times 10^{14} \mathrm{~W} / \mathrm{cm}^{2}$, and at $1 \times 10^{15}$ $W / \mathrm{cm}^{2}$ laser intensities is presented in Fig. 5-14. These data are absolutely calibrated, time-resolved measurements obtained with both the three-channel reflector/filter soft $x$-ray streak camera $(S X-1)$ and the five-channel metal-multilayer soft $\mathrm{x}$-ray streak camera $(\mathrm{SX}-6)$. The channel shapes of these two systems are shown in Figs. $3-4$ and $3-8$ respectively. Horizontal error bars on the data points in Fig. 5-14 represent the width of the various energy channels ald are typical of all points of a given photon-energy channel. Vertical error bars on the data points represent calculated calibration uncertainty of each of the two instruments and are typical of all data points corresponding to the individual instrument. The vertical extent of the reflector/filter $(S X-1)$ data points themselves represent the spread in data from numerous shots at each irradiation condition. Only a single Au target shot at each intensity was measured with $\mathrm{SX}-6$. The overall calibration of SX-6 data is obtained from comparisons with SX-1 on simultaneous measurements. Comparison between the three data sets is quite good except for the metal-multilayer (SX-6) points at $653 \mathrm{eV}, 737 \mathrm{eV}$, and $943 \mathrm{eV}$. These three channels were calibrated through a zomparison between the (SX-6) 653-eV metal-multilayer channel and the (SX-1) 700-eV reflector/filter iron channel. Apparently there is, as yet, an unresolved error in that calibration. The relative response between these three channels should be correct, however. 


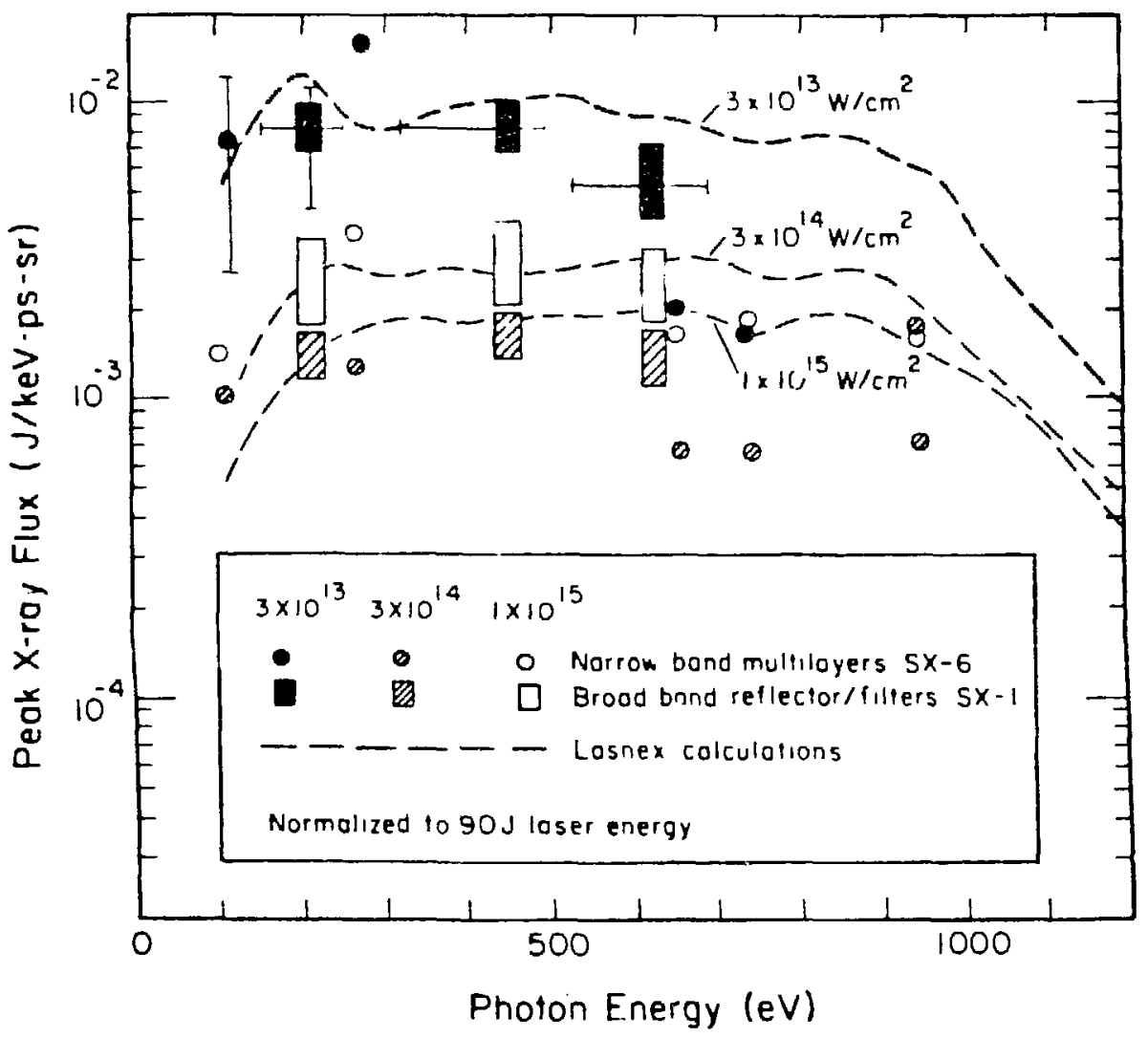

Fig. 5-14. Absolute Au spectral data from the reflector/filter system $(S X-1)$, from the metal-multilayer $x$-ray-interference-mirro 5 system (SX-6) and from LASNEX calcplations are compared at $3 \times 10^{13}$ $\mathrm{W} / \mathrm{cm}^{2}, 3 \times 10^{14} \mathrm{~W} / \mathrm{cm}^{2}$, and $1 \times 10^{15} \mathrm{~W} / \mathrm{cm}^{2}$ laser intensities. Higher laser intensities produce fewer therma $\perp$-rays and a flatter spectrum. The decrease in $x$-ray yield is probably due in part to reduced laser absorption. Also contributing to the reduced thermal $x$-ray $f l u x$ is a reduced conversion of light energy to thermal electron heating, e.g., supra-thermal electrons are more efficiently produced resulting in a spectral distortion with both a low and a high temperature component to the spectrum. The three highest narrow-band (SX-6) energy channels are calibrated together and appear to be low by a factor of $\mathrm{f} 2$. The observed and calculated spectra corcelate, well in shape and relative amplitudes. The measured $3 \mathrm{x}$ $10^{13} \mathrm{~W} / \mathrm{cm}^{2}$ spectrum falls off more rapidly at higher photon energies than predicted. Amplitude error bars shown are typical for each data set respectively. 
Also plotted in Fig. 5-14 are spectral emission shapes calculated using LASNEX for Au disks at the same three laser intensities. These three calculated spectral shapes are in reasonable agreenent with the measured data.

Principle features of these data are the flatness of the definitely non-Planckian spectral shape and the intensity dependence of the spectra. The SX-1 data points indicate nearly the same flat, spectral shape fron the three different irradiation intensities with a slightly more rapid fall-off for increasing photon energy at $3 \times 10^{13} \mathrm{~W} / \mathrm{cm}^{2}$. There is a distinct increase in $\mathrm{x}$-ray emission level with decreasing laser intensity.

\section{Peak Emission versus $Z$ and $I_{\text {laser }}$}

The peak emission level in the $200-\mathrm{eV}$ carbon channel of the reflector/filter soft $\mathrm{x}$-ray streak camera (SX-1) was observed to vary somewhat differently with laser irradiation intensity for different target materials. Figure 5-15 compares the absolute peak-flux levels from Au, Ti, and Be disk targets. The vertical lines represent spread in the data. The lines through the vertical data bars are intended to guide the eye. These trends in the data show peak emission dependences on the laser intensity of approximately $I^{-1 / 2}, I^{-1 / 3}$, $I^{-1 / 4}$ for $A u, T i$, and Be respectively.

\section{Layered Disk Energy-Transport Experiments}

An investigation of the energy-transport rate of thermal electrons through a thin Be coating to an $A l$ substrate was attempted in these experiments. A significant difference in $x$-ray emission efficiency of both bremsstrahlung and free-bound processes between the low-Z coating 


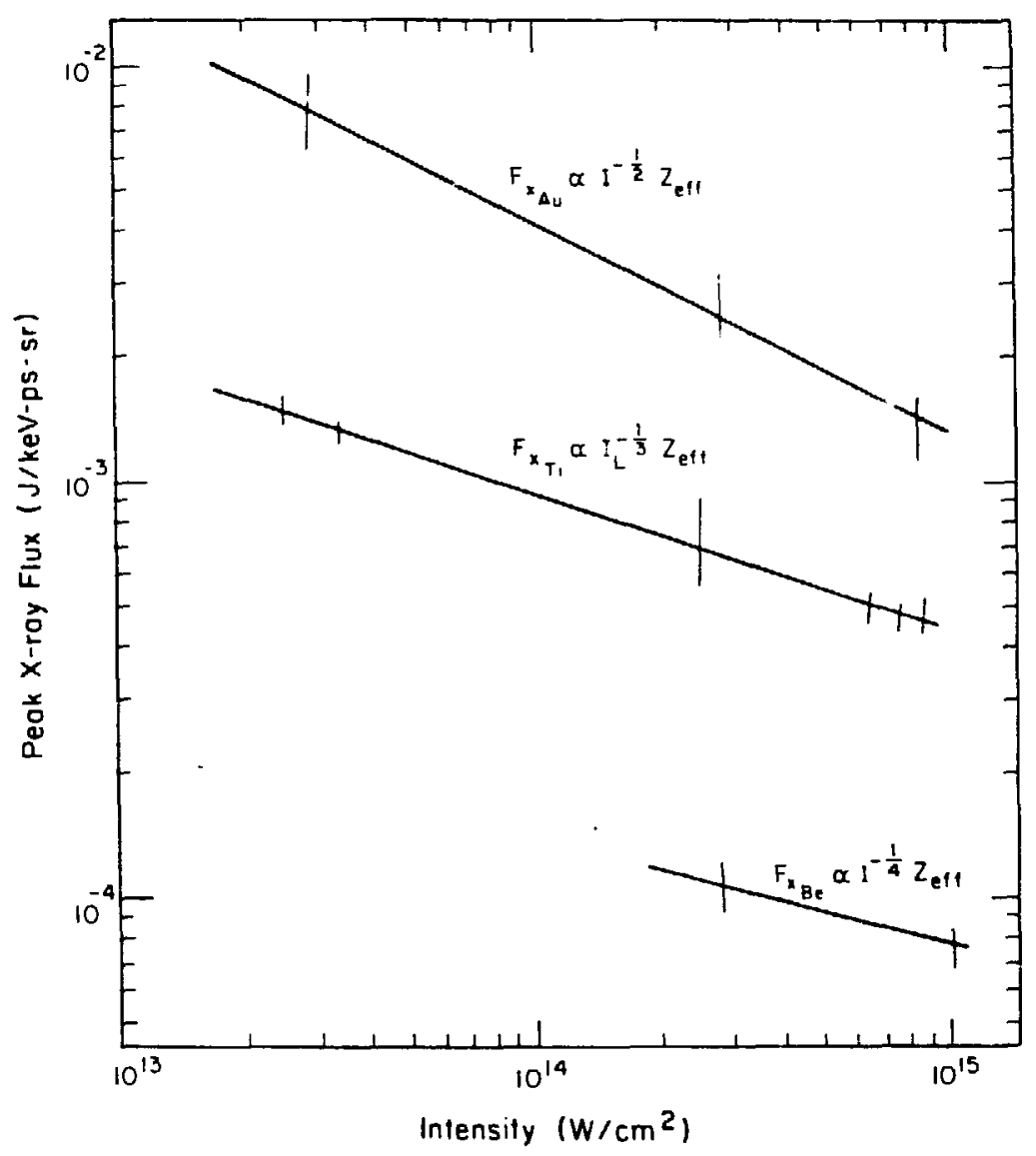

Fig. 5-15. Absolute values of peak flux in the 200-eV reflector/ filter carbon channel from $\mathrm{Au}, \mathrm{Ti}$, and Be disks as a function of laser intensity on target. All three materials decrease in emission level with rising laser intensity. Emission appears to decrease faster with higher target 2 . The vertical bars represent spread in the data. The lines drawn through the vertical data points are intended to guide the eye. The laser pulse-length and target parameters are the same as other data presented in this chapter. 
and the higher- $z$ substrate may be expected. Time-dependent change in the subkeV $x$-ray continuum level is then a measure of how rapidly and abruptly energy is transported to the Al substrate.

Disk targets of Al with front-side Be coatings of $0.25 \mu \mathrm{m}, 0.4 \mu \mathrm{m}$. $0.8 \mu \mathrm{m}$, and $5.6 \mu \mathrm{m}$, as well as pure $\mathrm{Al}$ and pure Be disks were irradiated at $3 \times 10^{14} \mathrm{~W} / \mathrm{cm}^{2}$ with $700-$ psec laser pulses. The targets were oriented with their surface normal $30^{\circ}$ east of the irradiation direction. $\mathrm{SX}-1$ and $\mathrm{SX}-6$ viewed these targets at $62^{\circ}$ and $47^{\circ}$, respectively, with respect to the surface normal (Fig. 5-2).

The sputtered-coating thicknesses were determined by an interferometric measurement of a witness piece. Position dependence in sputtering rates and the surface roughness of the substrates limited the thickness determination to $\pm 0.1 \mu \mathrm{m}$, a large uncertainty in some of the thinner coatings.

\section{Temporal Data Comparisons}

Typical temporal signatures in $200-\mathrm{eV}$ and $700-\mathrm{eV}$ energy bands. measured from layered disks irradiated at $3 \times 10^{14} \mathrm{~W} / \mathrm{cm}^{2}$, are shown in Figs. 5-16a and 5-17a respectively. Those figures show that the peak signal amplitude decreases and the pulse-width increases with Be thickness. The structure apparent on the pulses is typical of low-Z targets but not reproducible in detail. High-Z Au targets exhibit much smoother, Gaussian, temporal profiles even at low signal levels indicating that the structure is characteristic of $10 \mathrm{w}-Z$ disks and is not noise in the instrument. No burn-through signature is apparent under these experimental conditions.

Signal levels in the $700-e V$ (iron filtered) channel were below the thresiold for meaningful evaluation from targets with Be thicknesses 


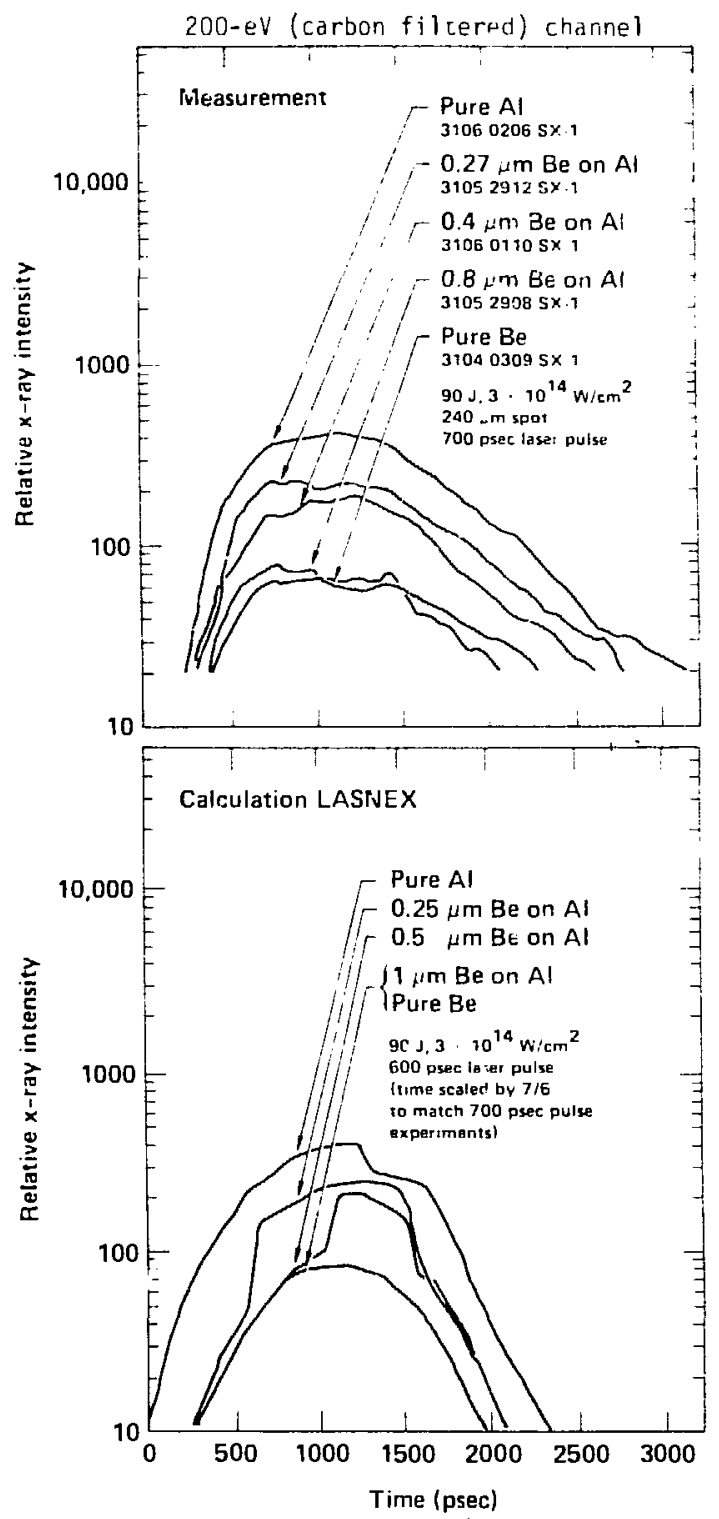

Fig. 5-16. X-ray emission histories, at $200 \mathrm{eV}$, from layered Be-on-Al disk targets. The upper box shows representative measurements from five different Be thicknesses in the carbon reflector/filter energy channel of $S X-1$. The lower box consists of LASNEX calculations of emission in a $175-\mathrm{eV}$ to $250-\mathrm{eV}$ energy bin from similar target geometries. Distinct, abrupt burn-through signatures are predicted from the one-dimensional computer simulations of thin-Be coated disk irradiations. This abrupt phenomena is not observed in the experiment. The calculations, which used a 600-psec Gaussian laser pulse, are dilated in time by $7 / 6$ to match the experimental laser pulse-length. However, the calculations exhibit much shorter emission pulse-lengths than do the measured experiments. In the upper box, the curves are positioned in time to have coincident rises. In the lower box, relative timing is given in the calculations. 


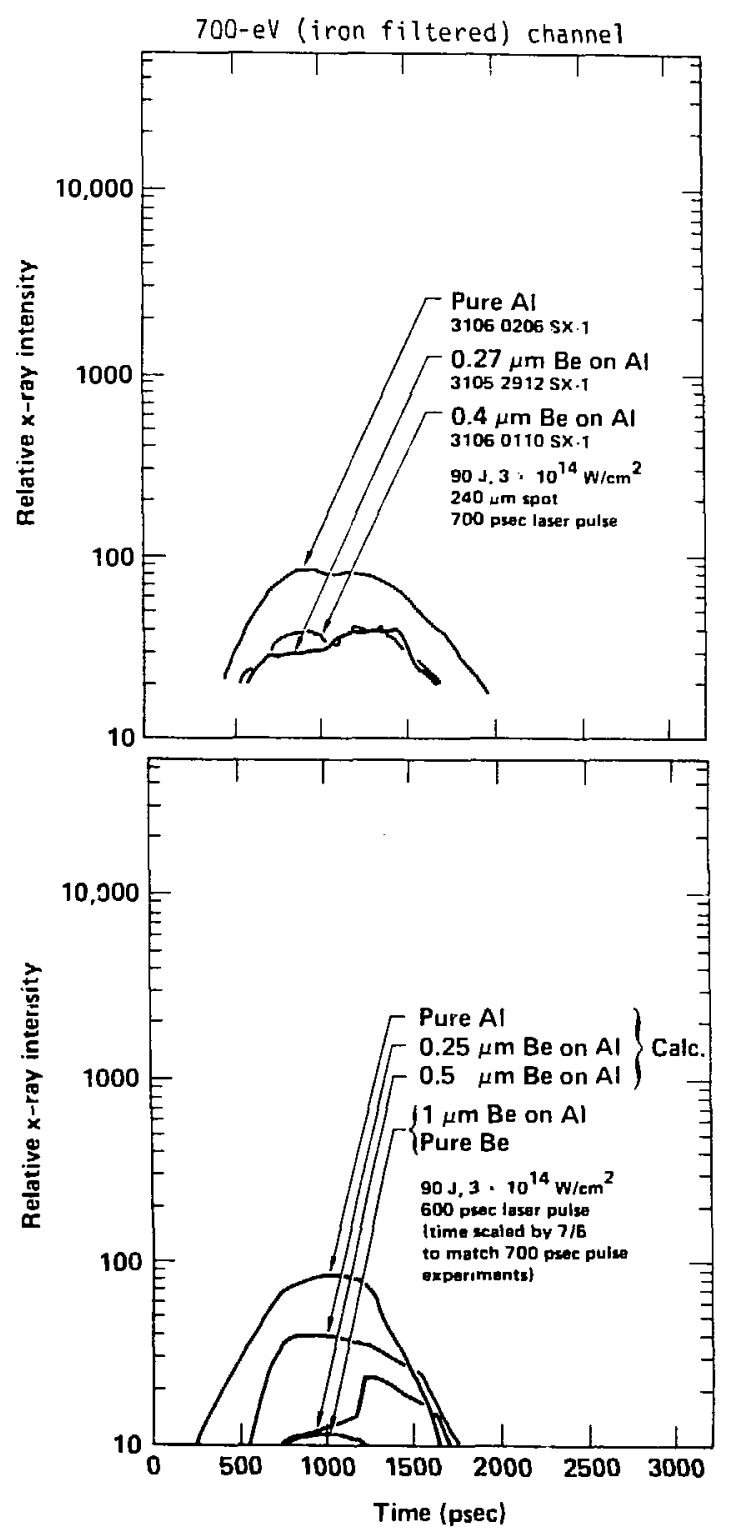

Fig. 5-17. X-ray emission histories, at $700 \mathrm{eV}$, from layered Be-onAl targets. The upper box shows measurements in the iron reflector/ filter channel of $\mathrm{SX}-1$. The lower box shows LASNEX calculations of emission in a 512-eV to 733-eV energy bin. Descriptive comments on Fig. 5-16 also apply here. 
of $0.8 \mu \mathrm{m}$ or greater. Emission from these thick Be targets was virtually the same in amplitude and overall pulse shape as the solid Be emission.

The calculated $x$-ray emission from LASNEX simulations of the experiment: in 200-eV carbon filtered and 700-eV iron filtered channels, identical to the spectral channel shapes of the measurements, ai presented in Figs. 5-16b and 5-17b respectively. The simulations were run with a 600-psec Gaussian laser pulse inst aad of the 700-psec Gaussian pulse of the experiments. A: before, time has been dilated by $7 / 6$ in the plots of LASNEX temporal shapes to match the experimental time scales. Although this correctior overestimates the pulse-width, the calculated $x$-ray emission is significantly shorter than that measured, for reasons which are only now being addressed in code physics.

The calculation predicts abrupt burn-through of a thermal wave to the Al substrate in $0.27-\mu \mathrm{m}$ and $0.4-\mu \mathrm{m}$ Be-coated disks. This results in a sudden increase in $x$-ray flux from the Al substrate at the burn-through time. For increasing Be thicknessses, the calculated burn-through time occurs later with respect to the turn-on time of the laser pulse. The burn-through time of a 1-um Be coating is longer than the emission pulse-width and is not seen in the simulated $x-r a y$ emission.

The differences between the measured and calculated pulse-shapes, particularly the lack of burn-through signature in the measurement, may result from inhomogeneities in the experiment. However, energy-depositing particles, with long mean-free paths compared to the expected thermal temperature gradient length and to the Be coating 
thickness, could also produce fairly prompt emission from the Al substrate. Time-integrated measurements at higher energies indicate that only a very small fraction of the absorbed energy goes into electrons which have ranges greater than $0.1 \mu \mathrm{m} .1,46,47$ Electrons of energies $10 \mathrm{keV}, 5 \mathrm{keV}, 3 \mathrm{keV}$, and $1 \mathrm{keV}$ have ranges 56 in $\mathrm{Be}$ $\left(1.85 \mathrm{~g} / \mathrm{cm}^{3}\right)$ of $2 \mu \mathrm{m}, 0.5 \mu \mathrm{m} 0.2 \mu \mathrm{m}$, and $0.03 \mu \mathrm{m}$ respectively. Perhaps the coronal temperature is higher than expected and some energy is transported to the substrate by freestreaming electrons rather than by a thermal diffusion wave at $3 \times 10^{14} \mathrm{~W} / \mathrm{cm}^{2}$.

\section{Peak Flux Comparisons}

A much closer correlation may be seen between the experiment and the simulations than that given by $x$-ray pulse shape comparisons in Figs. 5-16 and 5-17, by comparing the variation in relative peak amplitudes of $x$-ray emission for the various Be-coating thicknesses. The peak $x$-ray $f l u x$, both measured and calculated and at both 200-eV and $700-e V$, is plotted as a function of Be thickness in Fig. 5-18. The rate of flux fall-off is quite similar in both data sets. This fall-off corresponds to an exponential energy-transport attenuation length of $0.4 \mu \mathrm{m} \pm 0.1 \mu \mathrm{m}$ both in the experiment and in the simulation. The maximum depth of any detected heat penetration is $1 \mu \mathrm{m}$ in both cases.

Unlike the simulations shown above, the energy channels of these calculations are not shaped to match the instrument spectralsensitivity shapes (which remain the same), but are instead two energy bins closely related to the instrument channels which integrate emission bands between $175-\mathrm{eV}$ and $250-\mathrm{eV}$ and between $512-\mathrm{eV}$ and 733-eV. Here the calculated data set has been scaled so that the 


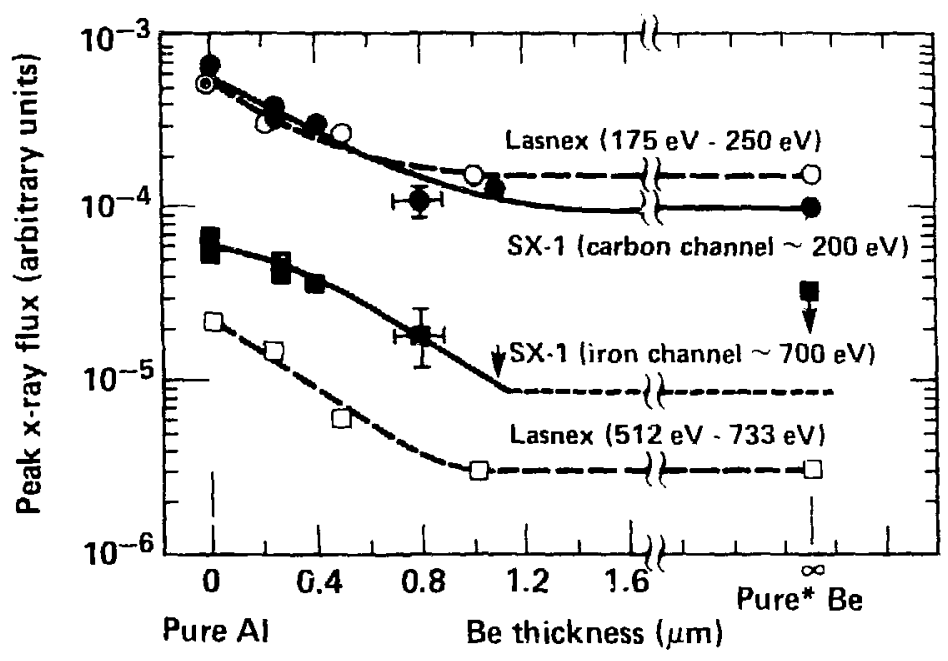

* "Pure Be" may include some high $Z$ impurities

Fig. 5-18. Peak x-ray flux as a function of Be coating thickness on Al falls at $1-\mu \mathrm{m}$ thicknesses to the level of solid Be experiments. Peak values of time-resolved data (shown in Figs. 5-16 and 5-17) from measurements and calculations in energy bands at $200 \mathrm{eV}$ and at $700 \mathrm{eV}$ are plotted. Solid daca points are measurements, open points are calculations, circles represent the 200-eV channel, squares represent the 700-eV channel. Error bars shown for the measurements are typical. The anomalously high measurement of pure Be emission at $700 \mathrm{eV}$ may be due to high-2 impurities in the forged Be target which was not as pure as the sputtered Be coatings. Arrows denote that emission levels are less than or equal to plotted amplitudes for given targets. 
amplitude of the $175-\mathrm{eV}$ to $250-\mathrm{eV}$ energy bin in the pure-Al calculation is equal to that of the similar, experimental 200-eV (carbon filtered) channel in the pure Al-disk measurement. The rest of the measured and calculated data points then self-consistently relate through this normalization. Some of the difference in peak-flux level between the high photon-energy channels of the two data sets may be due to differences in the energy ranges covered by the two 700-eV energy channels. Alternatively, there may be a difference between the temperature gradients used in the calculation and those which actually occur in the experiment. The anomalously high emission in the 700-eV (iron filtered) channel of the solid-Be measurement-data point is probably due to high-Z impurities found in the solid-Be disk targets. The solid, cast-Be disks were fabricated from a different Be source than the sputtered Be coatings on the layered Jisks.

\section{Section Summary}

A first effort has been made to time-resolve thermal, subkeV energy-transport phenomena from Be-on-Al layered targets. Several distinctive features were observed. Distinct burn-through signatures did not occur in the time-resolved $x$-ray pulse-shapes. Instead of an abrupt step-up in $x$-ray emission from a thermal wave encountering the Al substrate, the $x-r a y$ emission increased fairly smoothly to a peak and then decayed fairly smoothly. The peak value of the thermal $x$-ray emission did decrease with coating thickness to the soiid-Be disk emission level at $\sim 1-\mu m$ coating thicknesses.

Significant differences are apparent between LASNEX simulations of the layered-target emission pulse-shapes and the observed pulse- 
shapes. With the best understanding available of target physics and conditions, the one-dimensional calculations predict abrupt burnthrough of the thermal heat front through $0.25-\mu \mathrm{m}$ and $0.4-\mu \mathrm{m}$ Be thicknesses, while this effect is not observed. Some areas for concern about possible differences between the experimental conditions and the calculational assumptions are that the experiment may have had twodimensional non-uniformities of beam intensity, coating characteristics, or energy-transport rates. The calculation is one-dimensional and therefore perfectly uniform in the lateral direction. Longer range particles than expected by LASNEX may also have resulted in early $x$-ray emission from the Al substrate in Figs. 5-16a and 5-17a. Further investigations of the contributions of experimental non-uniformities in the lateral direction will be importar to clarify these issues. Higher-quality coatings and beam profiles are obtainable. Spatially resolved and time-resolved thermal $x-r a y$ measurements can be done. More sophisticated experimental designs as described above will make the measurements more precise.

\section{Conclusion}

A new dimension is opened for thermal diagnostic analysis of laser heated plasmas through the time-resolved, subkeV, spectrally resolved $x-$ ray measurement techniques presented in this chapter. Thermalelectron energy-transport rates may be directly observed through carefully controlled layered-target experiments. Energy-transport and energy-balance models may be contrained and indirectly checked through comparisons between time-resolved $x$-ray measurements and computer simulations of $x-r a y$ emission from similar experiments. In 
the experiments described above, significant energy transport in Be is limited to $\$ 1-\mu m$ in 700 psec. Deposition of energy to the substrate was not seen to be abrupt, but rather occurs throughout the laser irradiation period.

New data on $x$-ray emission pulse-length and peak-flux level variations with both laser intensity and target $Z$ are presented. Non-Planckian Au spectral data below $1 \mathrm{keV}$ are shown.

Conclusions to be drawn from this work include observations that more effort can and should profitably be expended both in making more refined and better-controlled energy-transport measurements and in resolving questions raised about the fidelity of physical models used in LASNEX.

Specific experimental refinements which should be considered are improved uniformity of both laser intensity on the target and the layers of the target itself. Time-resolved spectrometers tuned to observed, selected, unambiguous emission lines rising out of the continuum will provide more analytic, tractable data. Such an experiment may be designed to give the material temperatures and burn-through of several layers in multi-layered targets. 


\section{References}

1. M. D. Rosen, D. W. Phillion, V. C. Rupert, W. C. Mead, W. L. Kruer, J. J. Thomson, H. N. Kornblum, V. W. Slivinsky, G. J. Caporaso, M. J. Boyle, and K. G. Tirsell, Phys. Fluids 22, 2020 (1979).

2. G. B. Zimmerman and W. L. Kruer, Comments Plasma Phys. 2, 85 (1975); G. B. Zimmerman, Lawrence Livermore National Laboratory UCRL -74811 (1973).

3. M. H. Key, J. D. Kilkenny, C. L. S. Lewis, and S. A. Veats, Rutherford Laboratory Report, Annual Report to the Laser Facility Committee 1980, RL-80-026, p. 4-6 (1980); P. Cunningham, C. L. S. Lewis, A. K. Roy, J. M. Ward, T. Goldsack, J. D. Kilkenny, S. Veats, B. McGowan, L. Pina, M. H. Key, W. T. Tones, and P. T. Rumsby, Rutherford Laboratory Report, Annual Report to the Laser Facility Committee 1981, RL-81-040, p. 4-10, (1981).

4. R. C. Malone, R. L. Mc Croy, and R. L. Morse, Phys. Rev. Lett. 34 , $721(1975)$.

5. J. S. Pearlman and J. P. Anthes, Appl. Phys, Letters 27, 581 (1975).

6. R. A. Haas, W. C. Mead, W. L. Kruer, D. W. Phillion, H. N. Kornblum, J. D. Lindl, D. MacQuigg, V. C. Rupert, and K. G. Tirsell, Phys. Fluids 20, 322 (1977).

7. B. Yaakobi and T. Bristow, Fhys, Rev. Lett. $\underline{38} 350$ (1977).

8. J. T. Larsen and D. T. Attwood, unpublished.

9. D. T. Attwood, IEEE J. Quantum Electron. QE-14, 909 (1978).

10. D. Shvarts, C. Jablon, I. B. Bernstein, J. Virmont, and P. Mopra, Nucl. Fusion 19, 1457 (1979).

11. J. Dellettrez and E. B. Golman, Laboratory for Laser Energetics report, 36 (1976).

12. R. J. Mason, Phys. Rev. Letters 42, 239 (1979); R. J. Mason, Los Alamos Scientific Laboratory LA-78-2776 (1978).

13. J. R. Albritton, I. B. Bernstein, E. J. Valeu and E. A. Williams, Phys. Rev. Lett. $\underline{39}, 1536$ (1977).

14. K. A. Brueckner and R. S. Janda, Nucl. Fusion 17, 1265 (1977).

15. W. L. Kruer, Comments Plasma Phys. Controlled Fusion 5, 69 (1919). 
16. L. Spitzer and R. Harm, Phys. Rev. 89, 977 (1953); L. Spitzer, Jr., Physics of Fully Ionized Gases (Interscience, New York. 1965).

17. B. H. Ripin, P. G. Burkhalter, F. C. Young, J. M. McMahon, D. G. Colombant, S. E. Bodner, R. R. Whitlock, D. J. Nagle, D. J. Johnson, N. K. Winsor, C. M. Dozier, R. D. Bleach, J. A. Stamper, and E. A. McClean, Phys Rev. Lett. 34, 1313 (1975).

18. J. A. Stamper, K. Papadopoulous, R. N. Sedan, S. O. Dean, E. A. McLean, and J. M. Dawson, Phys. Rev. Lett. 26 1012 (1971).

19. D. G. Colombant and N. K. Winsor, Phys. Rev. Lett. 38,697 (1977).

20. C. E. Max, W. M. Manheimer, and J. J. Thomson, Phys. Fluids 21 . $128(1978)$.

21. D. W. Forslund, J. Geophys. Res. 15,17 (1970).

22. R. J. Bickerton, Nucl. Fusion 13, 457 (1973).

23. R. C. Malone, R. L. McCrory, and R. L. Morse, Phys. Rev. Lett. 34 721 (1975).

24. W. M. Manheimer, Phys. Fluids, 20, 265 (1977).

25. D. R. Gray, J. D. Kilkenny, M. D. White, P. Blyth, and D. Hull, Phys. Rev. Lett. 39, 1220 (1977).

26. S. J. Gitomer and D. B. Henderson, Phys. Fluids 22, 364 (1979).

27. I. B. Bernstein, C. E. Max, and J. J. Thomson, Phys. Fluids 21 , 905 (1979).

28. A. R. Bell, R. B. Evans, and D. J. Nicholas, Fhys. Rev. Lett. 46 , $243(1981)$.

29. D. Shvarts, T. Delettrez, R. L. McCrory, and C. P. Verdon, Phys. Rev. Lett. 47, 247 (1981).

30. R. Balescu, seminar at Lawrence Livermore National Laboratory (1981).

31. R. J. Mason, Phys. Rev. Lett. 47, 652 (1981).

32. K. Mizuno, R. B. Spielman, J. S. DeGroot, and W. M. Bollen, Proc. Joint Conf. 4 th Kiev Intern. Plasma Theory and 4 th Intern. Congress Waves and Instabilities in Plasmas (Nagoya, Japan, 1980), p. 306; K. Mizuno, J. S. DeGroot, D. A. Rasmussen, F. Kehl, and Wee Woo, Lawrence Livermore National Laboratory report, Laser Program Annual Report-1980, UCRL-50021-80, Vol. 2 p. 3-67 (1980). 
33. H. A. Baldis and C. J. Walsh, Bull. Am. Phys. Soc, 26, 954, 973, 1012 (1981).

34. K. Esterbrook, Phys. Rev. Lett. 47, 1396 (1981).

35. D. R. Gray, J. D. Kilkenny, M. S. White, P. Blyth, and D. Hull, Phys. Rev. Lett. $\underline{39}, 1270$ (1977).

36. A. A. Offenberger, A. Ng, and L. Pitt, Phys. Rev. Lett. $\underline{40}, 873$ (1978).

37. W. L. Kruer, private communication.

38. J. A. Stamper and B. H. Ripin, Phys. Rev. Lett. 34 138 (1975); J. A. Stamper, E. A. Mclean and B. H. Ripin, Phys. Rev. Lett. 40 , 1177 (1978).

39. A. Raven, O. Willi, and P. T. Rumsby, Phys. Rev. Lett. 41,554 (1978).

40. D. T. Attwood, Lawrence Livermore National Laboratory reports: Laser Program Annual Report-1975, UCRL-50021 (1976), p. 428; Laser Program Annual Report-1977, UCRL-50021-77, Vol. 1, p. 3-52.

41. W. Seka, J. L. Schwob, C. Breton, J. Appl. Phys. 42, 315 (1971).

42. B. Yaakobi and T. C. Bristow, Phys. Rev. Lett. 38,350 (1977).

43. F. C. Young, R. R. Whitlock, R. Decoste, B. H. Ripin, D. J. Nagel, J. A. Stamper, J. M. McMahon, and S. E. Bodner, Appl. Phys. Lett. 30, 45 (1978).

44. D. L. Banner, W. C. Mead, E. M. Campbell, and W. L. Kruer, Lawrence Livermore National Laboratory report, Laser Program Annual Report - 1979, UCRL-50021-79, Vol. 2, p. 6-12 (1979).

45. J. C. Weisheit, C. B. Tarter, J. H. Scofield, and L. M. Richards, J. Quanı. Spect. Radiat. Transfer 16,659 (1976).

46. K. G.Tirsell, H. N. Kornblum, and V. W. Slivinsky, Bull. Amer. Phys. Soc. 23, 807 (1978); Lawrence Livermore National Laboratory report, UCRL-81478 (1979).

47. P. H. Y. Lee, K. G. Tirsell, E. M. Campbell, F. Ze, and R. E. Turner, Lawrence Livermore National Laboratory report, 1980 Laser Program Annual Report, UCRL-50021-80, pp. 7-10, 7-22.

48. N. M. Ceglio, M. Roth, and A. M. Hawryluk, Low Energy X-Ray Diagnostics-1981, edited by D. T. Attwood and B. L. Henke (AIP, New York, 1981), p. 290.

49. Ya. B. Zeldovich and Yu. P. Raizer, Physics of Shock Waves and High Temperature Hydrodynamic Phenomena (Academic Press, New York, 1966), Vol. 1, p. 258. 
50. Ibid. This equation may be 'obtained from a straightforward manipulation of the material in Section 5-1 pp. 261-266.

51. Ibid. p. 290.

52. E. M. Campbell, private communication.

53. G. L. Stradling, T. W. Barbee, Jr., B. L. Henke, E. M. Cambpell, and W. C. Mead, Low Energy X-Ray Diagnostics-1981, edited by D. T. Att wood and B. L. Henke (AIP, New York, 1981), p. 292.

54. G. McClellan, P. H. Y. Lee, and G. Caporaso, Phys. Rev. Lett. 44 , $658(1980)$.

55. G. L. Stradling, R. L. Kauffman, E. M. Campbell, M. D. Rosen, and W. C. Mead, Bull. Amer. Phys. Soc. 25, 885 (1980); also Lawrence Livermore National Laboratory report, UCRL-84749 (1980).

56. These ranges are extrapolated from M. J. Berger and S. M. Seltzer, National Aeronautics and Space Admin. Repori, NASA SP-3012 (1954). Berger and Seltzer provide range values for electron energies of $10 \mathrm{keV}$ to $1000 \mathrm{MeV}$ electrons. 


\author{
APPENDIX \\ STRE $i K$ CAMERA DATA HANDLING, \\ REDICTION, AND ENALYSIS
}

\title{
Introduction
}

Analytic conversion of the streaked data on film to useful quantitative data may be accomplished through careful application of film sensitometry, computer-controlled densitometry, computer imageprocessing and noise suppression techniques. This Appendix will describe the procedures in use at LLNL. 1

\section{Eilm Sensitometry}

Film density response to the image-intensifier signal may be calibrated. A proper calibration, which includes film non-linearities, spectral characteristics, and reciprocity effects, allows quantitative film-density - to - intensifier-signal intensity conversion. The P-20 phosphor, used in the image-intensifiers on the soft $x$-ray streak cameras, fluoresces in the yellow-green, $4600-\AA$ - to - 6490- $\AA$ region of the spectrum. The persistence of $P-20$ is two miliseconds.?

\section{Sensitometer Configuration}

These characteristics are simulated on an EG\&G Mark VI xenon-flash sensitometer using a No. 93 green Wratten filter. With $\pm 3 \%$ reliability, film is exposed to this source through a calibrated, $10^{5}$ range, Kodak exposure stepwedge in $10^{-3}-\mathrm{sec}$ flashes. This calibration exposure wedge is placed upon each film after data exposure. Such a procedure eliminates uncertainty in film response due to variations in day-to-day film development conditions and to variatious in film batch characteristics. 
Film Processing

After exposure of the stepwedge scale, the film is developed. Generally, Kodak Royal-X Pan $f i l m$ is used for high sensitjvity and large dynamic range. The dynamic range of this film is enhanced by development in DK-50 developer for 4.5 minutes at $72^{\circ} \mathrm{F}$ with nitrogen burst agitation. Figure $\mathrm{A}-1$ is an example of an RXP data image including the calibrated step wedge.

\section{Computer-Controlled Densitometry}

Digital densitometry of the recorded data is done on a Photometric Data Systems (PDS) computer-controlled micro-densitometer. For soft. x-ray streak records we typically employ a 100-um square aperture and 100-um steps in scanning the data. This aperture dimension represents the resolution-element size in the data and gives a fairly rapid scan speed. Care should be exercised in the POS aperture geometry and the sampling size used when full, instrument-limited resolution is critical. 3 Sampling theory requires $3-5$ samples per resolution element in such cases. As discussed in tha text, the soft x-ray streak camera has better resolution capability than is required by the measurements presented here. Thus a $100-\mu m$ square aperture is adequate.

Noise suppression is addressed later, in the image-processing stage rather than during densitometry, through data-averaging techniques. Data averaging during image processing is more convenient and flexible than the equivalent scanning techniques using a larger-area rectangular window, and/or overlapping scan steps.

In addition to the streak data, the step-wedge of each film is densitometered. A density-vs-position digital file for each data set is 
transferred via magnetic tape to the CDC-7600 computers where the data manipulation procedures are performed.

\section{Computer Inage Processing and Noise Suppression}

Manipulation of the mirco-densitometered, streaked-image data is handled by a program ATTWOOD* which runs as a macro-system under the general analysis code MATHSY. Both ATTWOOD and MATHSY were designed and written by Giles Peterson at LLNL. The results of each step in the analysis are shown graphically after each operation. Hard copy of each display may also be obtained.

D-vs-logE Polynomial Fit

ATTWOOD initially fits an n-degree ( $n$ is typically 7 ) polynomial to the stepped density-vs-log exposure data (figure A-2). A monotonically rising Eilm response is required sor unambiguous data conversion. If the polynomial fit to the data exhibits a hump on the toe or a drop at the shoulder--or other non-monotonic features--the analyst may drop data points at the toe or shoulder or may change the value of $n$ to produce a more satisfactory fit. The characteristic curve of the film is calculated from the approved fit and a data file of density-vs-log exposure is written. Both the stepped and polynomial fit exposure curves are shown in figure A-3.

\footnotetext{
*The macro program ATTWOOD was initially designed for D. T. Attwood for use in $x$-ray streak camera data processing. Some evolution of the program has occured as image processing and noise suppression needs have developed further.
} 
Streak Data Display

Analysis of data from a relatively high-flux $i\lrcorner$ disk irradiation, recorded on the $S X-1$ reflector/filter instrument, will be used in this section of the Appendix as an example. The streaked image of this data with the exposure wedge is shown in figure A-l. The three bright streaks, from left to right, are the 700-eV (iron filtered) channel, the $500-e V$ (vanadium filtered) channel, and the $200-e V$ (carbon filtered) channel. These channel positions are reversea in the digital contour images. Time in both cases proceeds from bottom to top.

The film-density data of the streak image is displayed as a twodimensional isodensity contour plot in figure A-4. Dotted and solid curves correspond to density variations of 0.2 and 1.0 , respectively. The array of density data is spatially averaged over an integral number of rows and columns to conserve effort in the computer. A group of data points $r$ rows high and $c$ columns wide are averaged to nake a single data point with an $r x c$ reduction in data handling operations. Of course, there is an accompanying reduction in resolution of the data and sometimes a noticeable discreteness introduced. As in this data, the features across the spatial dimension of the image generally consist of several broad, uniform channels. Some averaging of columns across tre spatial dinension of the photocathode has no effect on the data quality and substantially decreases processing time. Generally, full resolution in the time direction is desirable and a more sophisticated averaging technique than data compression is used for noise smoothing.

The working data set may be expanded to the required resolution and simultaneously truncated in image area by excluding uninteresting rows 
or columus from the borders of the array in the next operation (figure A-5). With the density data array appropriately truncated and with the desired averaging in the spatial dimension, the density data is then converted to log-intensity versus position data through the film characteristic-response data file. This is a nonlinear conversion. Care must be exercised in interpreting low-density data because the density representing the fog level on the toe of the characteristic curve, indicated in figure $A-2$, correspond to a large range of log-intensity values. A resulting noise exhancement is observed for densities oscillating about this average fog level, as seen at late time in Fig. A-6.

The converted data is exponentiated to the base ten and ploted as iso-intensity contours in figure A-6. Now dotted and solid isointensity contour curves correspond to factors of 2 and 10 in relative intensity, respectively. The working digital data array in memory is retained as log-intensity values for further operations.

Row and Column Averaging

A specific number of rows or number of columns of the data array may be averaged to give an average signal level as a function of position on the photocathode or as a function of time. Figure A-7 shows a fairly large section of rows near the peak of the pulse specified for averaging; and figure A-8 gives the time-average signal level as a function of position across the photocathode.

When there are a number of columns occupied by a uniform spectral channel, significant signal smoothing is obtained by averaging over the extent of the channel. Figure $A^{-9}$ defines the averaging limits for three channeis. Figures A-10 through A-12 are the time-dependent signal 
levels in the three channels, averaged over the spatial extent of each channel. The instrument sweep-speed is used to convert from position to time coordinates. Figure A-13 is a composite of the temporal profiles from the three channels. Note that a relative intensity level of 10 corresfonds to the average fog level of the D-logE characteristic curve and that interpretation of lower signal levels in figures A-10 through A-13 must be done only with great caution. Averaging over larger sections of the data set does reduce noise fluctuations. However, when the resulting signal levels are near the toe of the film response, the error bars are larger and, of course, the resolution is degraded.

\section{Data Smoothing}

Frequently, low signal levels are accompanied by significant levels of high frequency shot noise and intensifier induced granularity. In addition, the spatial extent of a channel may be small ias with the 2-mm wide Sx-6 channels) and thus not provide much help in terms of spatial averaging across the channel. A data smoothing option may be selected in which a running average is performed on the intensity array in the time direction. A number of rows (time steps) $r: R_{a}, R_{a+1}, \ldots, R_{a+r}$,

are averaged to form a single row, $R^{\prime} a^{\text {. Then }} \mathrm{R}_{a+1}, \mathrm{R}_{a+2}, \ldots, \mathrm{R}_{a+r+1}$ are averaged to form row $R^{\prime} a+l$ and so on through the entire array. The resulting averaged array has been filtered to reduce noise of timescales less than the time-scale corresponding to $r$ rows. If a row corresponds to $100 \mathrm{\mu m}$ in the time direction, at $2136 \mathrm{psec} / \mathrm{mm}$, its width represents $\imath 14 \mathrm{psec}$.

The effectiveness of this smoothing operation is shown in the next group of figures. A low-signal-level, noisy data streak from a Be-disk 
shot is used in these examples. The data, again, was taken with the SX-l reflector/filter soft $x$-ray streak camera. The channel configurations are the same as those shown in the last data set. Figure A-14 is the streaked image with time going from bottom to top. Only the 200-eV (carbon filtered) channel has significant signal levels.

Figures $\mathrm{A}-15$ and $\mathrm{A}-16$ are the exposure calibration D-vs-logE plots similar to figures $A-2$ and $A-3$. Figure $A-17$ is the iso-density contour plot of the streak image. Density values barely reach a level of 1 . The converted iso-intensity contours are displayed in figure A-18.

A time average of the position-dependent photocathode response over the pulse duration is specified in figure A-19 and displayed in figure A-20. In this case, the time window over which the data is averaged is large enough that fairly reliable, average signal levels are obtained in figure A-20, even in the low signal level, noisy channels. These vanadium and iron filtered channels are formed by averages of sharp spikes, resulting from low-level density variations near fog density, as will be seen in figures $\mathrm{A}-23$ and $\mathrm{A} 24$.

Spatial averages across the channels specified in figure A-21 give a distinct but noisy temporal pulse-shape for the carbon channel in figure $A-22$. The other two channels shown in figures $A-23$ and $A-24$ are very noisy. Meaningful signal levels can only be obtained at the sacrifice of most temporal detail and do not contain meaningful data, as in figure $A-20$.

Smoothing operations, which filter temporal structure of 60 psec, $120 \mathrm{psec}$, and $180 \mathrm{psec}$, by doing a running average over periods of four rows, eight rows, and twelve rows, respectively, are performed on the 
200-eV (carbon filtered) channel and are displayed in figures A-25, A-26, and A-27. Marked reduction in "grass" is seen with 60-psec smoothing and it is gone after 120-psec smoothing. Smoothing of 180 psec begins to suppress features of the pulse shape.

More sophisticated smoothing techniques are available which are designed to minimize distortion in the pulse shape being smoothed. 4 Such techniques should be considered for use in more marginal situations and when temporal noise fluctuations and pulse length are of comparable duration. 


\section{References}

1. Film sensitometry, development, and densitometry proceedures used here were established by and are maintained by claude Dittmore of the Technical Photography group in L-Division of LLNL.

2. Optical Characteristics of Cathode Ray Tube Screens, JEDEC publication No. 16-C (Electronic Industries Association, Washington, D.C., 1975) P. 102 .

3. E. K. Hodson and J. Canada, private communication.

4. E. K. Hodson, D. R. Thayer, and C. Franklin, IEEE Trans. Acoust., Speech, Signal Processing ASSP-29, 854 (1981). 


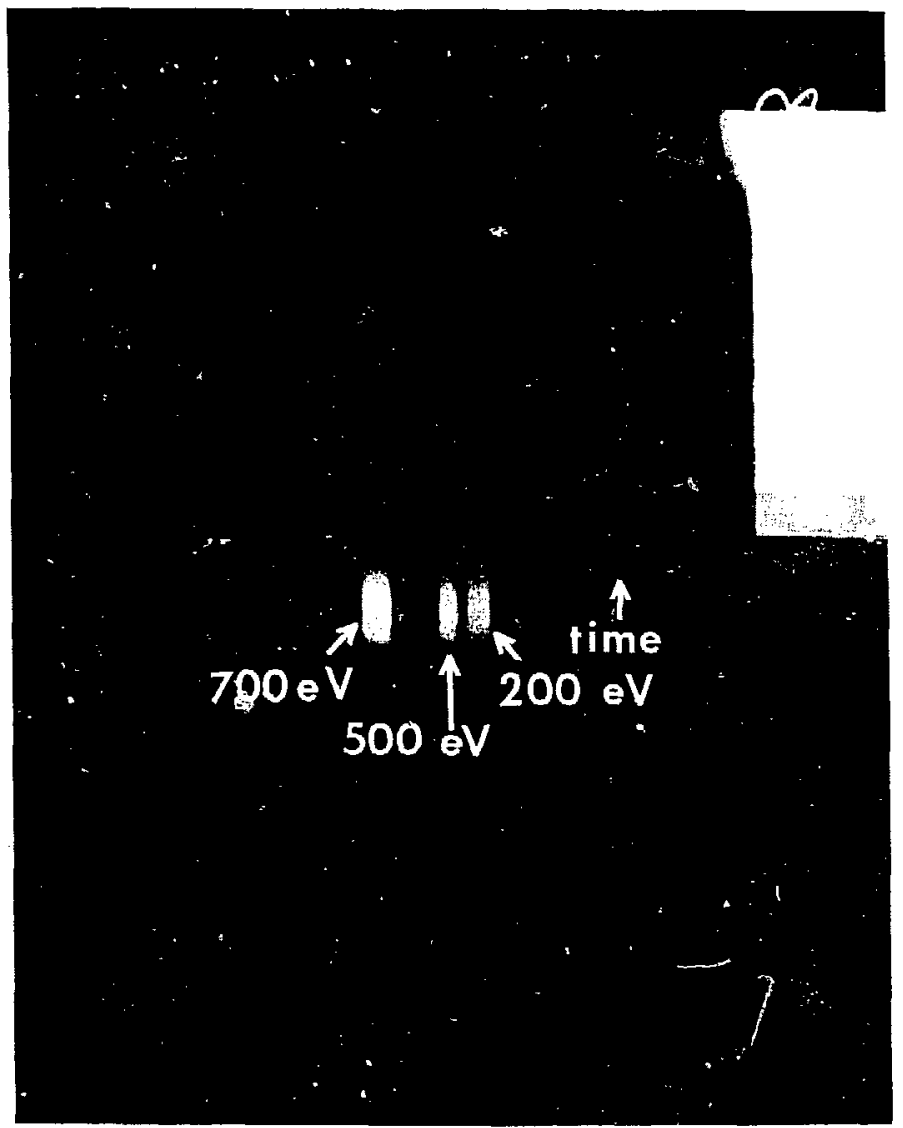

Figure A-1. Streaked image of an $x$-ray pulse from an Au disk target on 4"x5" RXP film. The exposure step wedge is on the right edge of the film. The three intense data channels from left to right are the $700-e v$ (iron filtered) channel, the 500-eV (vanadium filtered) channel, and the 200-ev (carbon filtered) channel. The two lower intensity level strips flanking the two latter channels are merely more heavily filtered sections of the adjacent channels. Time goes from bottom to top. This data was taken with a sweep speed of $134 \mathrm{psec} / \mathrm{mm}$. 


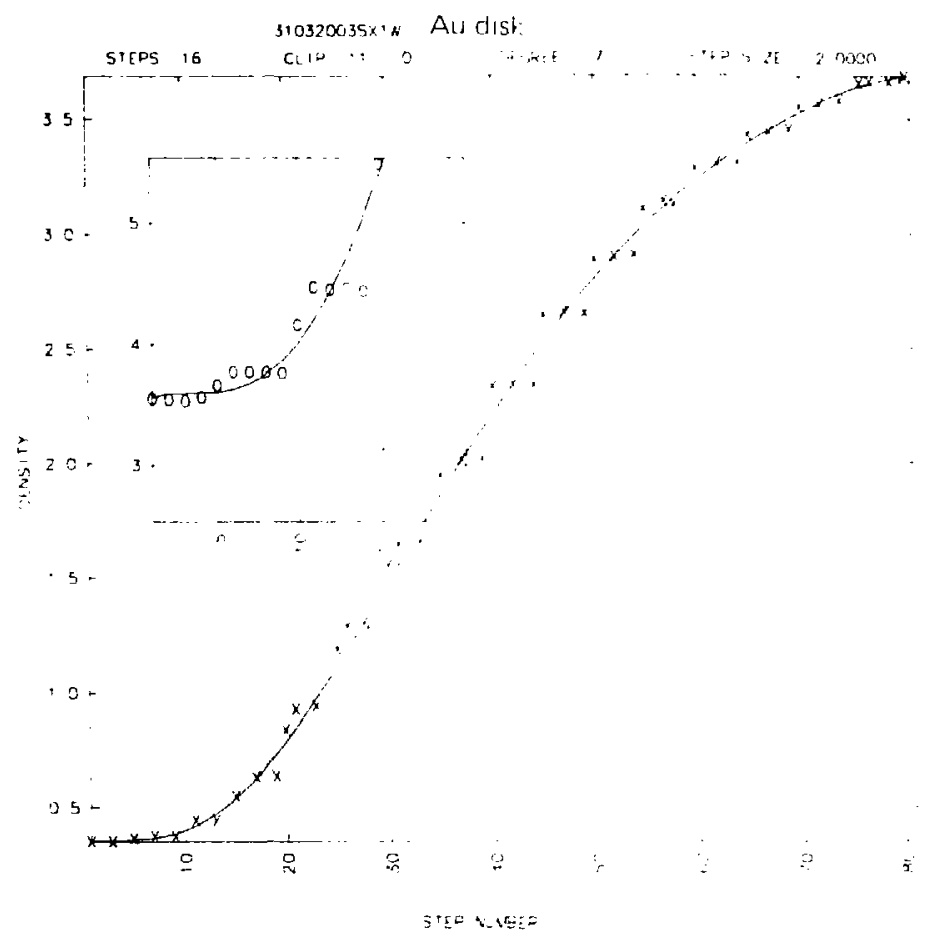

Figure A-2. A seven-degree polynomial fit to the exposure step-wedge densities. The step wedge varies by factors of two. Eleven data points have been truncated from the toe to obtain a monotonically increasing fit. The insert is a detailed view of the fit at the toe to allow detection of ripples in the fitting curve. 


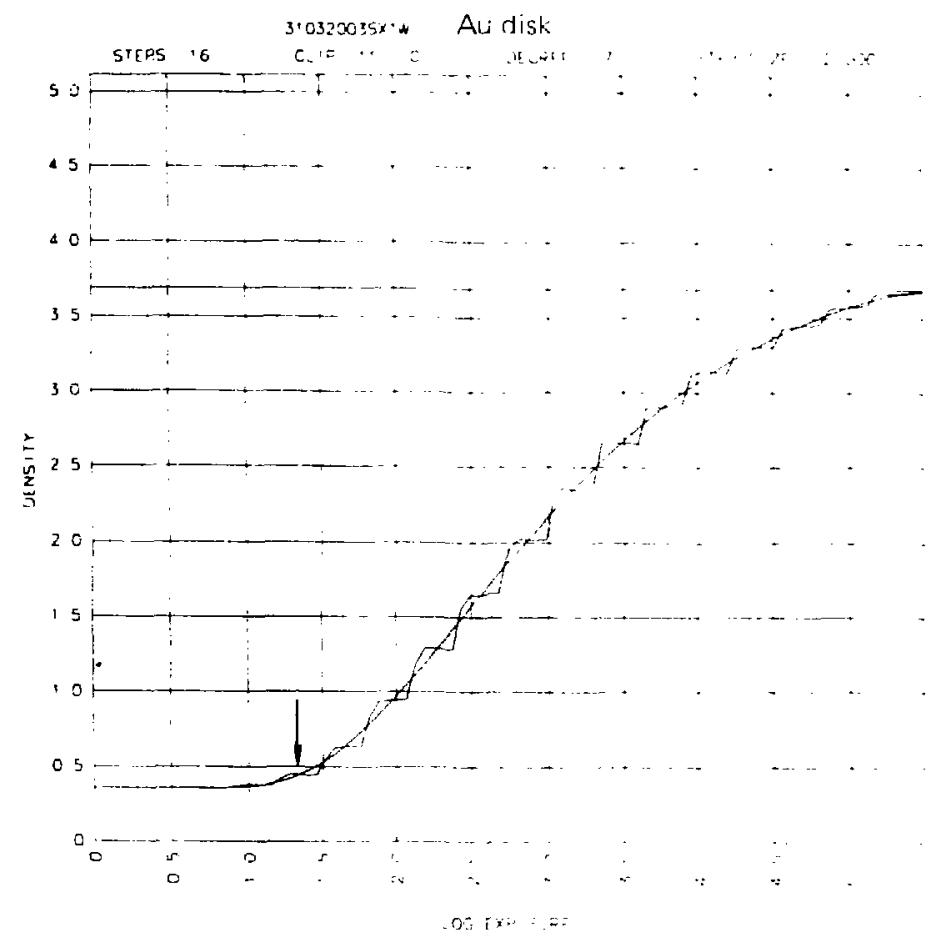

Figure A-3. Density-vs-log exposure polynomial fit to the step wedge from figure A-2. The arrow indicates a minimum density level below which intensity conversion must be performed with extra care. 


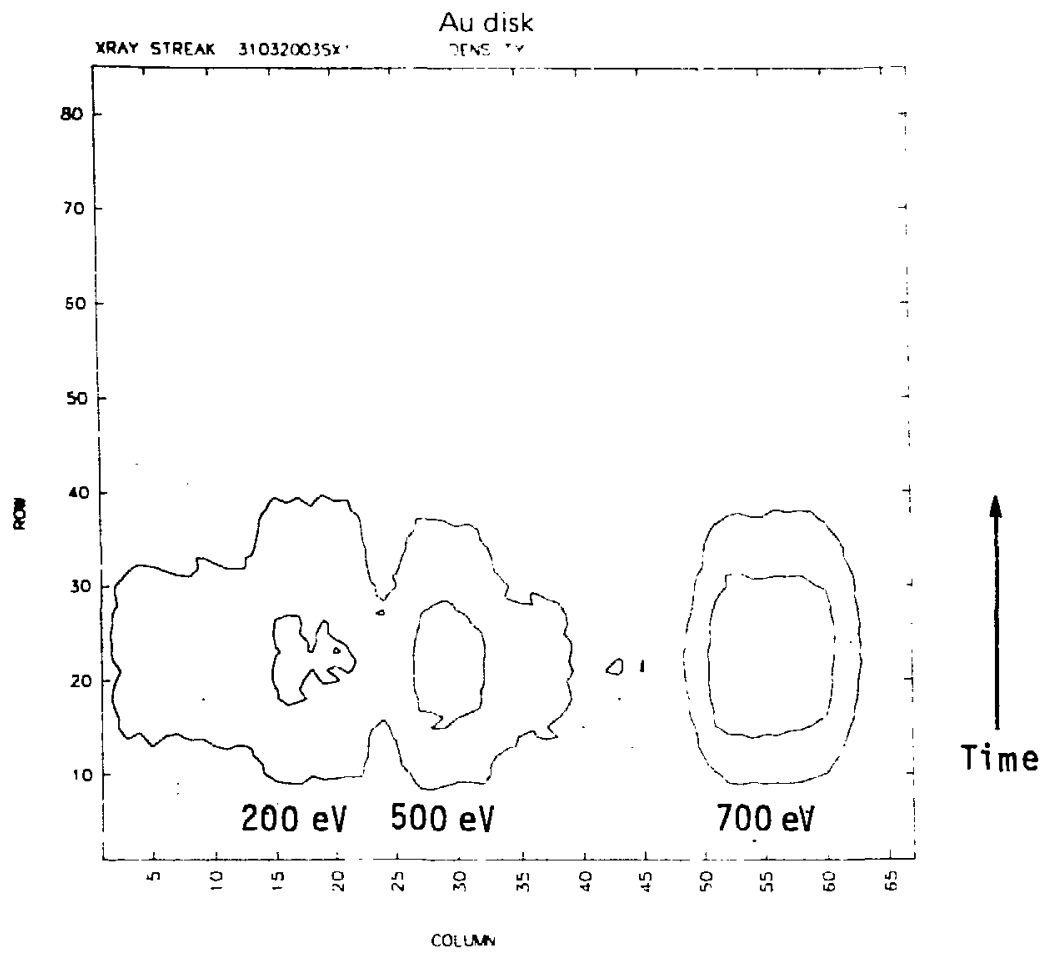

Figure A-4. Iso-density contours of the data of figure A-1. Dotted and solid curves represent density variations of 0.2 and 1.0 , respectively. The horizontal axis has been reversed from the previous figure so that the 200-eV (carbon filtered) channel is on the left. Time goes from bottom to top. 


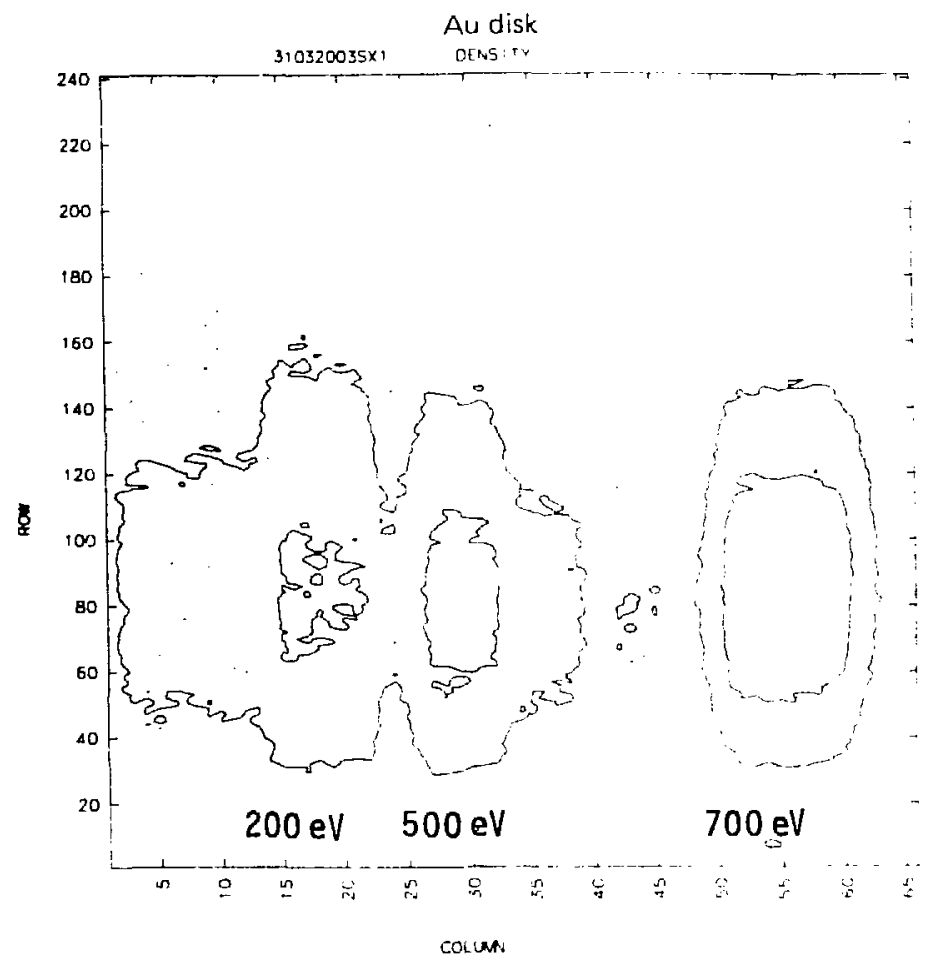

Figure A-5. The iso-density contour with late-time rows eliminated and maximum resolution in the time direction. In the spatial dimension one column represents three compressed columns of data. 


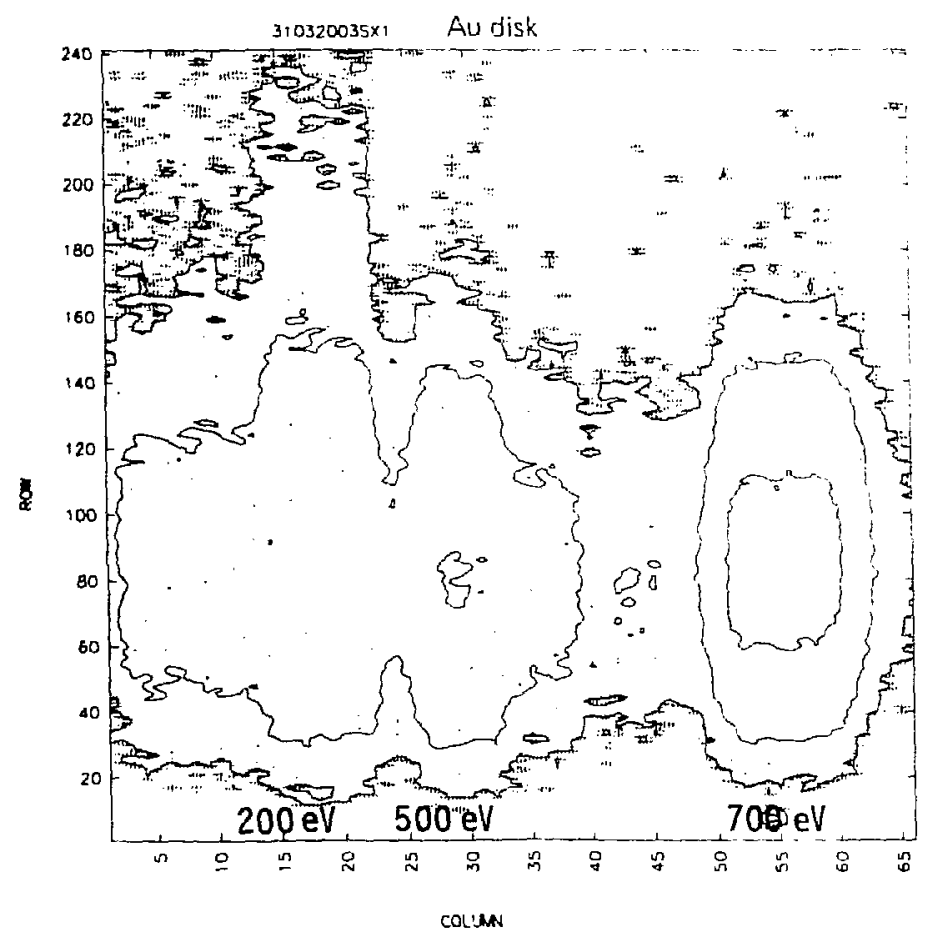

Figure A-6. The previous figure converted to iso-intensity contours through the characteristic density-vs-log exposure function of figure A-3. Dotted and solid contours represent differences in intensity of 2 and 10 , respectively. 


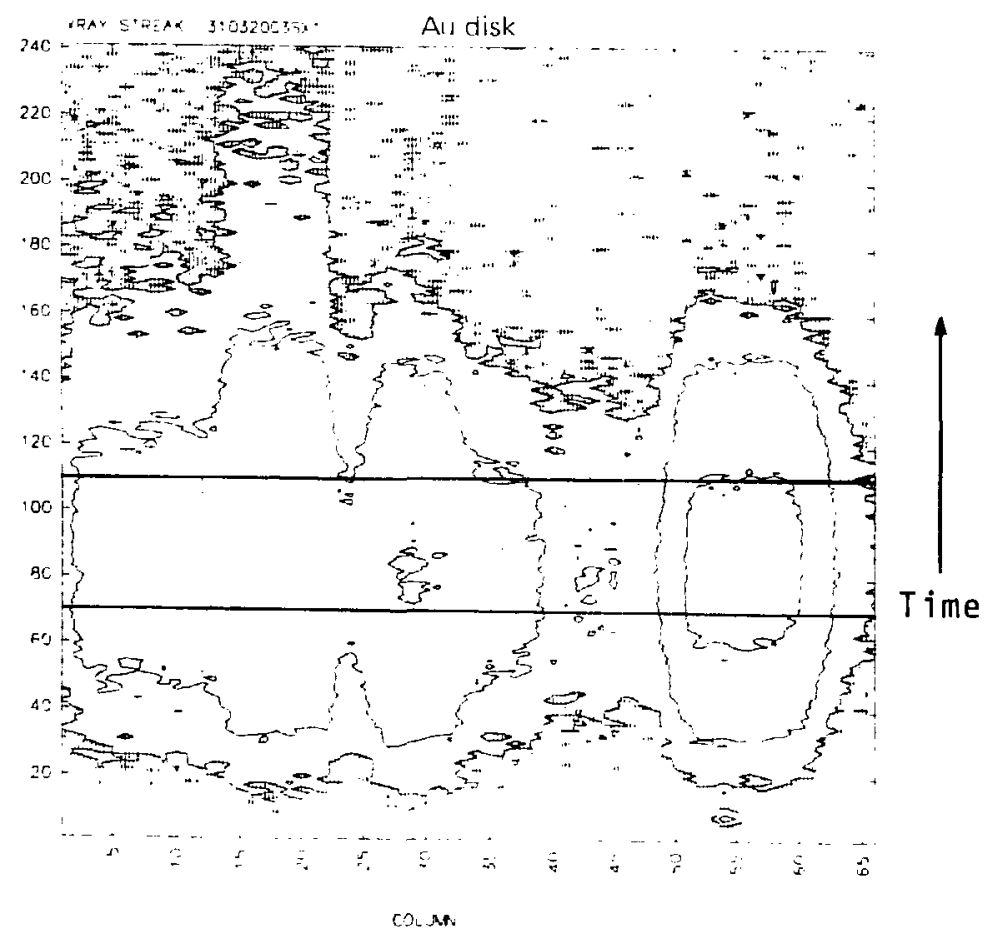

Figure A-7. The previous figure with rows 70 through 110 designated for time averaging. 


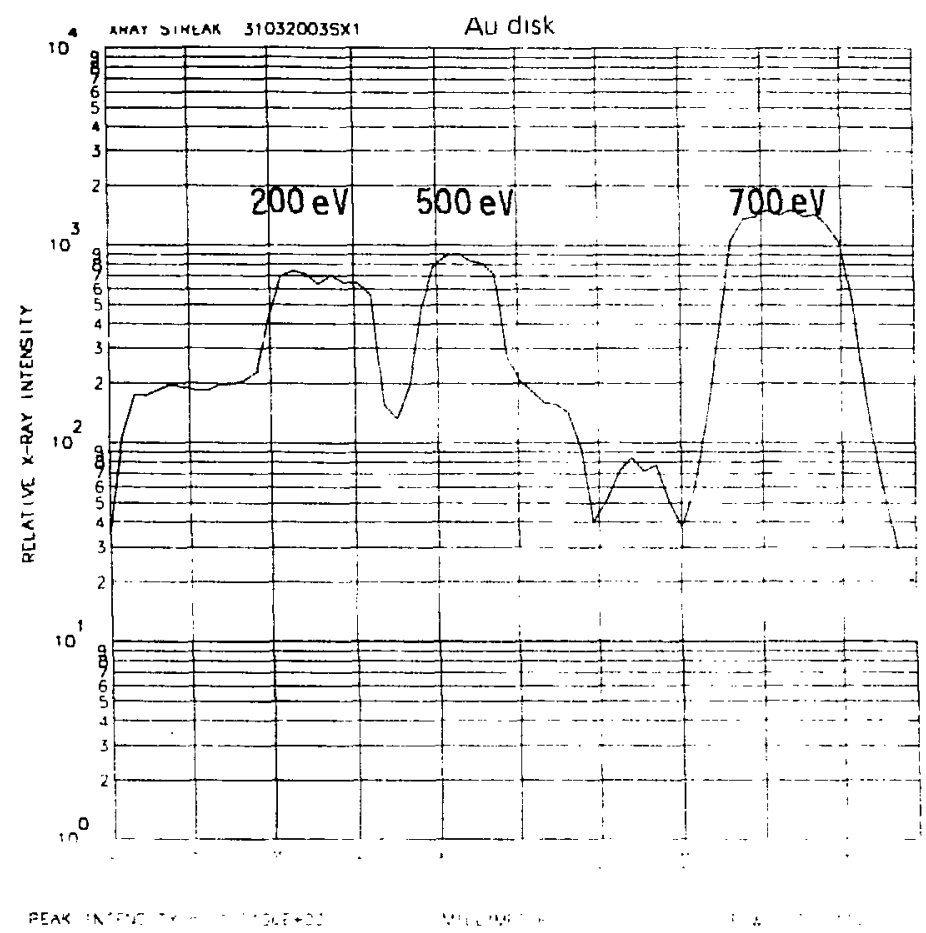

Figure A-8. Time average of intensity values for rows 70 through 110 across the spatial extent of the photocathode. 


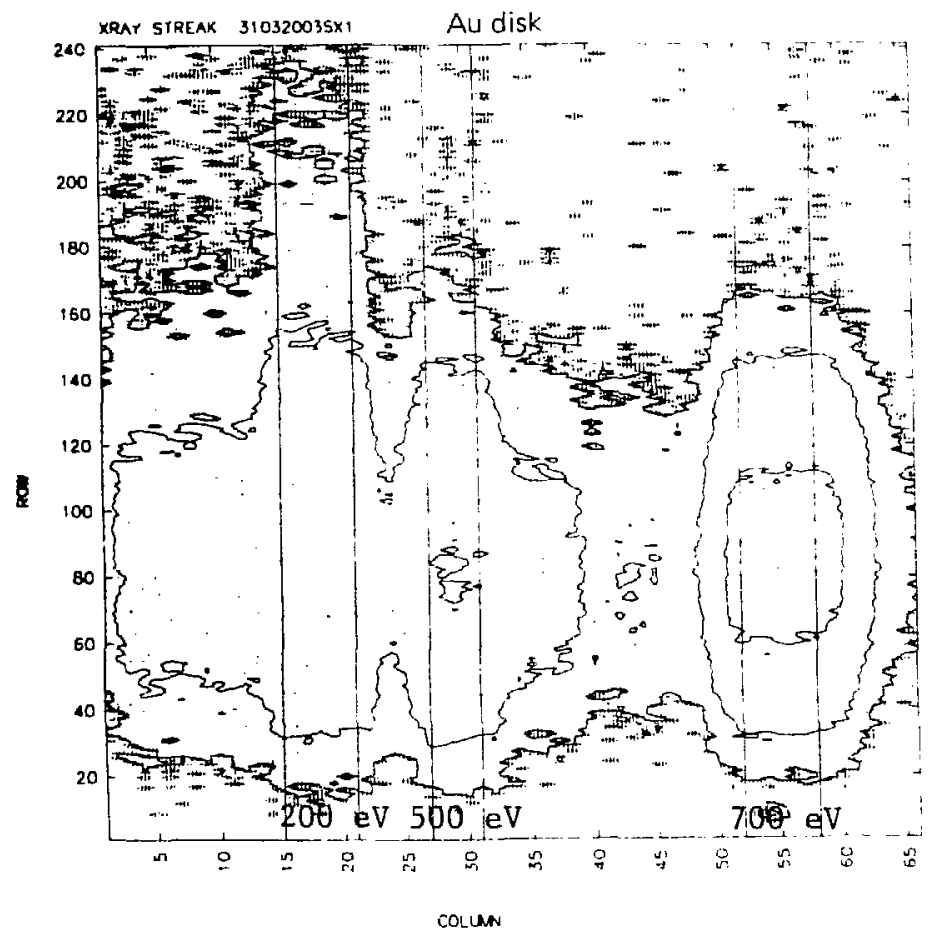

Figure A-9. Figure A-6 with the extent of the three channels to be spatially averaged marked. Averaging is to be done between rows 15 and 21, 27 and 31 , and between 52 and 58 . 


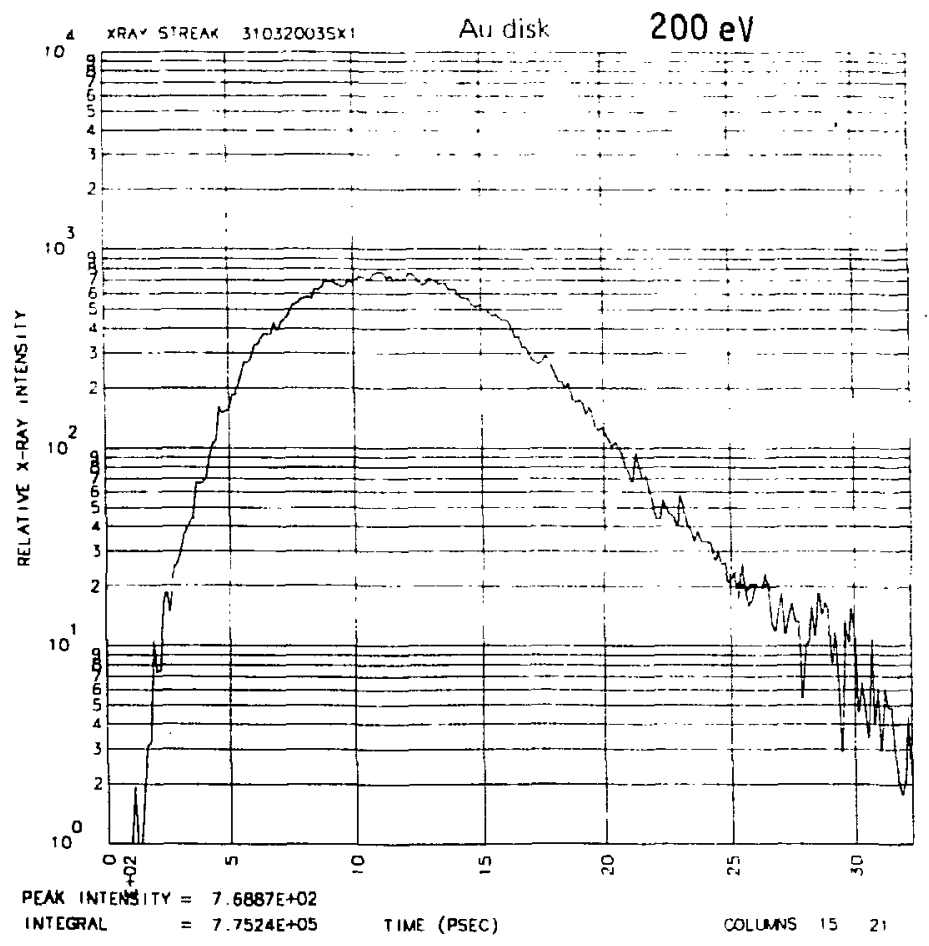

Figure A-10. The average value of the carbon filtered channel as a function of time over 3.2 nsec. 


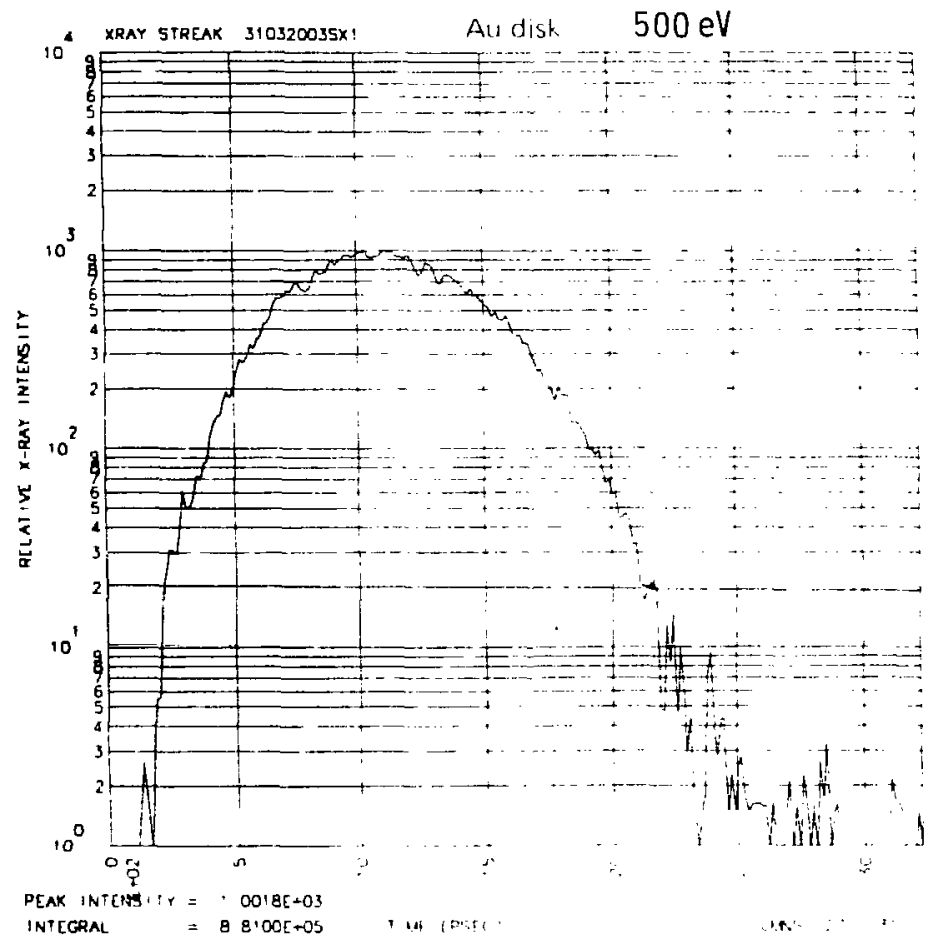

Figure A-11. The average value of the vanadium filtered channel as a function of time over $3.2 \mathrm{nsec}$. 


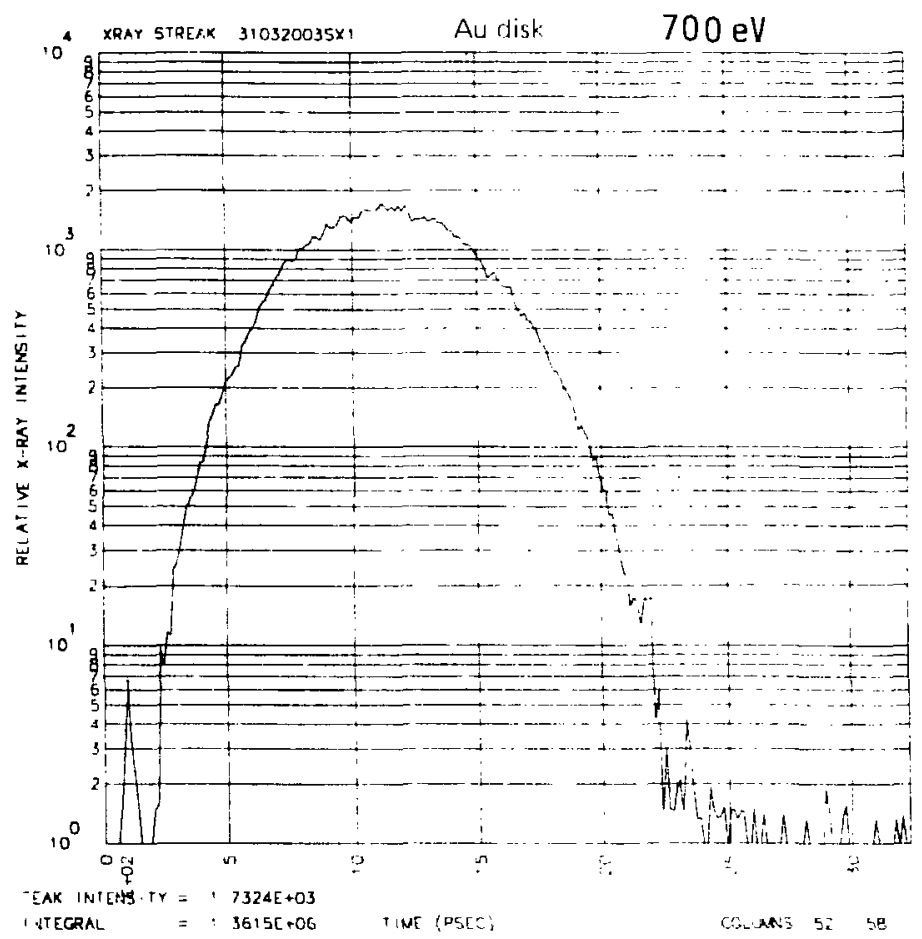

Figure A-12. The average value of the iron filtered channel as a function of time over $3.2 \mathrm{nsec}$. 


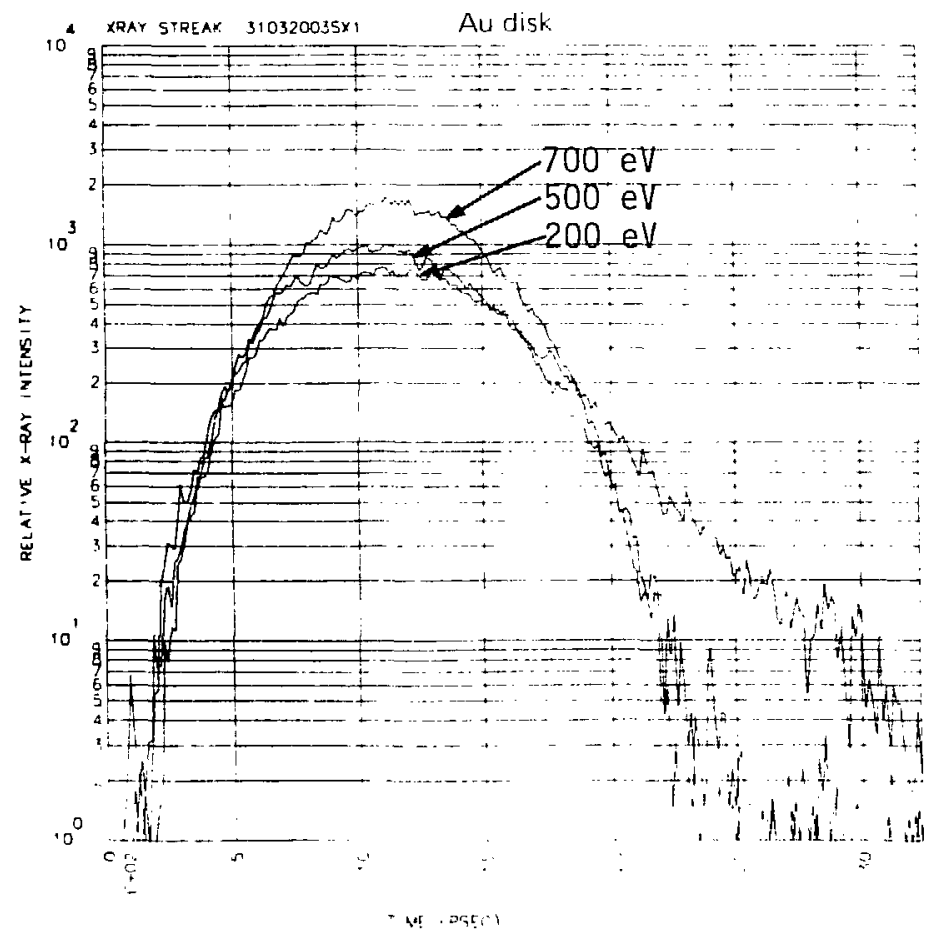

Figure A-13. A composite of the three energy channels. Amplitudes of the three channels are not absolutely scaled with respect to each other. 


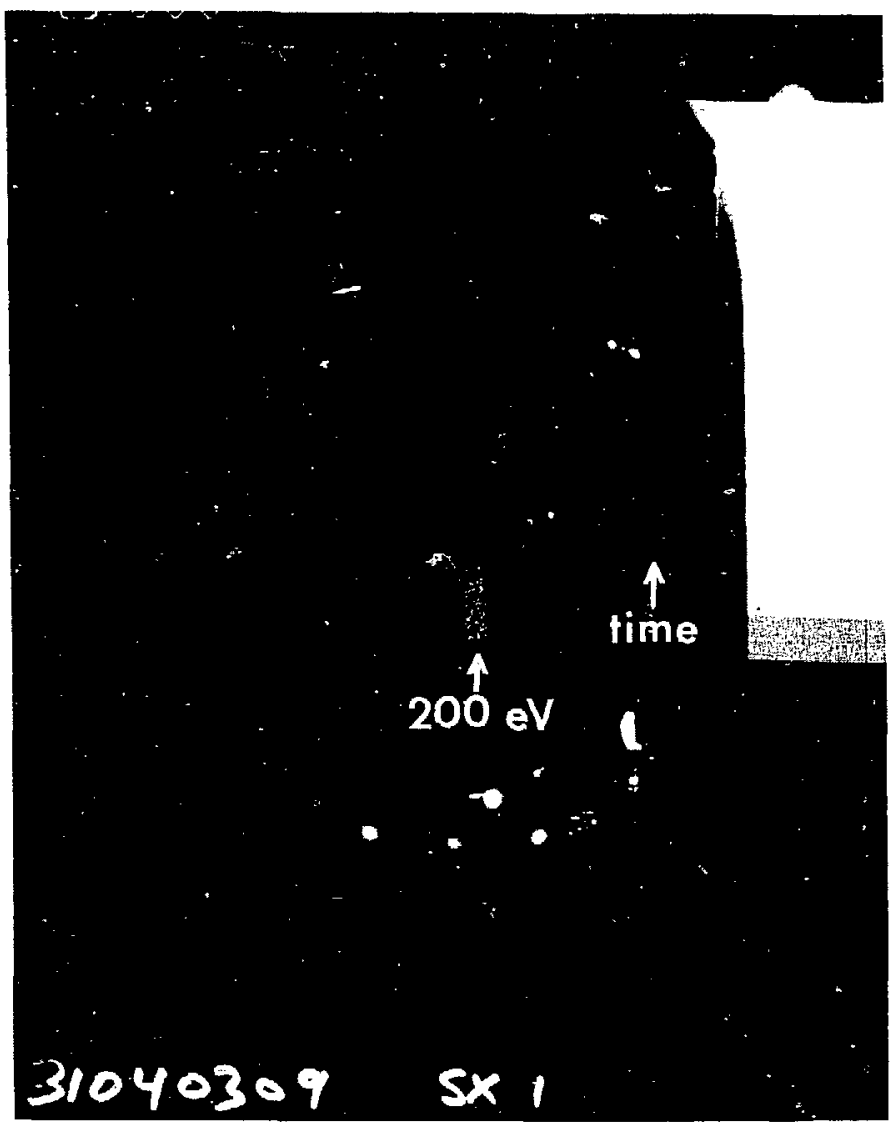

Figure A-14. Streaked image of low-level, noisy data from a Be disk irradiation on $4^{\prime \prime} \times 5^{\prime \prime} \mathrm{RXP} F \mathrm{i}^{1} \mathrm{~m}$. The $200-\mathrm{eV}$ (carbon filtered) channel is apparent. Time goes from vottom to top. This data was taken with a sweep speed of $134 \mathrm{psec} / \mathrm{mm}$. 


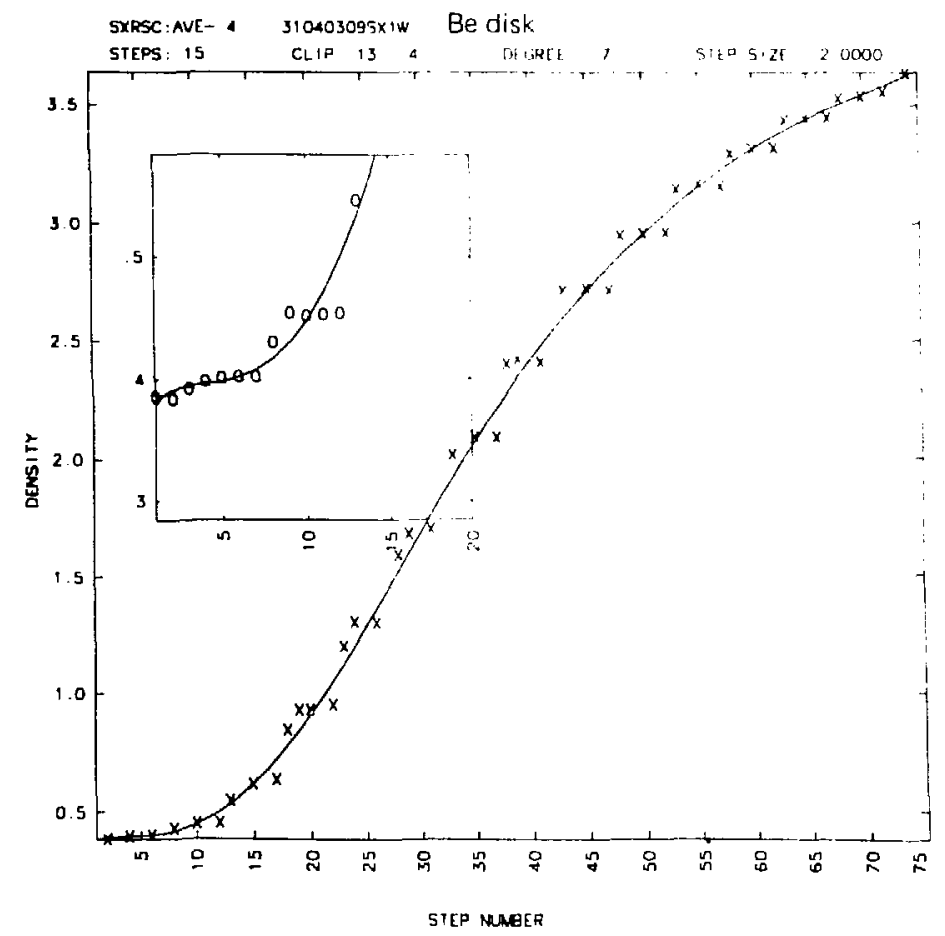

Figure A-15. Polynomial fit to step wedge of figure A-14. 


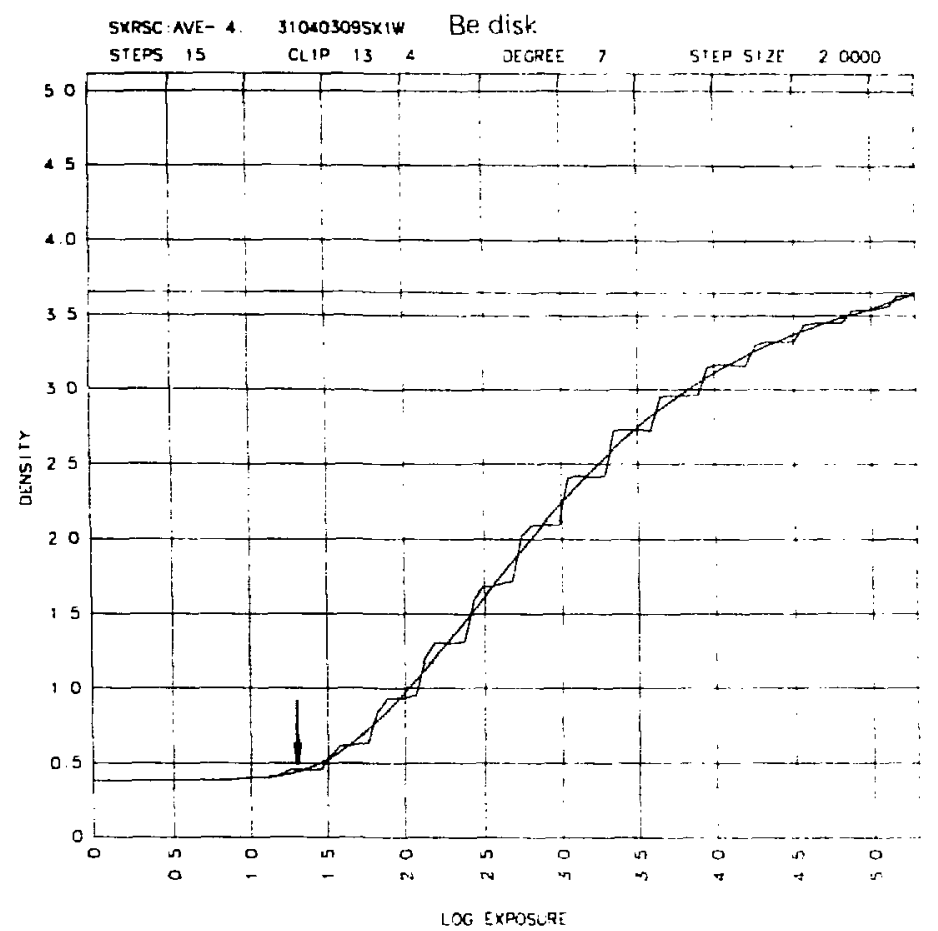

Figure A-16. Density-vs-log exposure for the film of figure A-14. 
Be disk

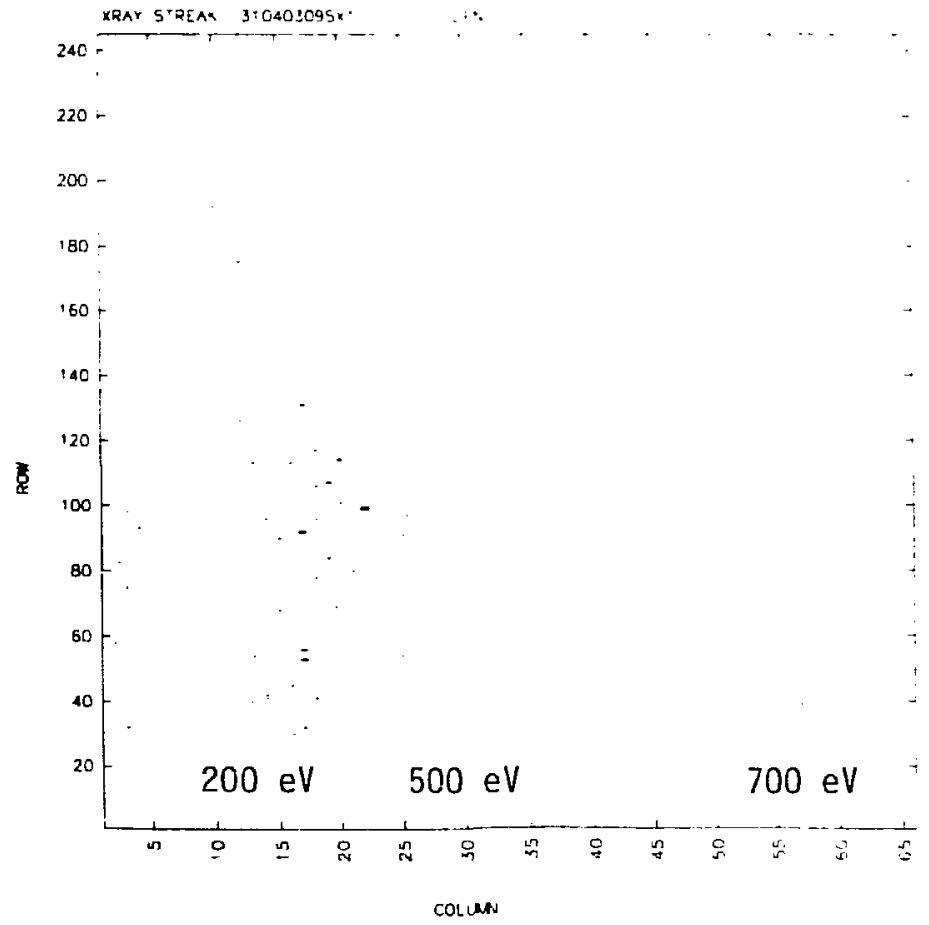

Figure A-17. Iso-density contour plot of the Be-data streaked image from figure A-14. 


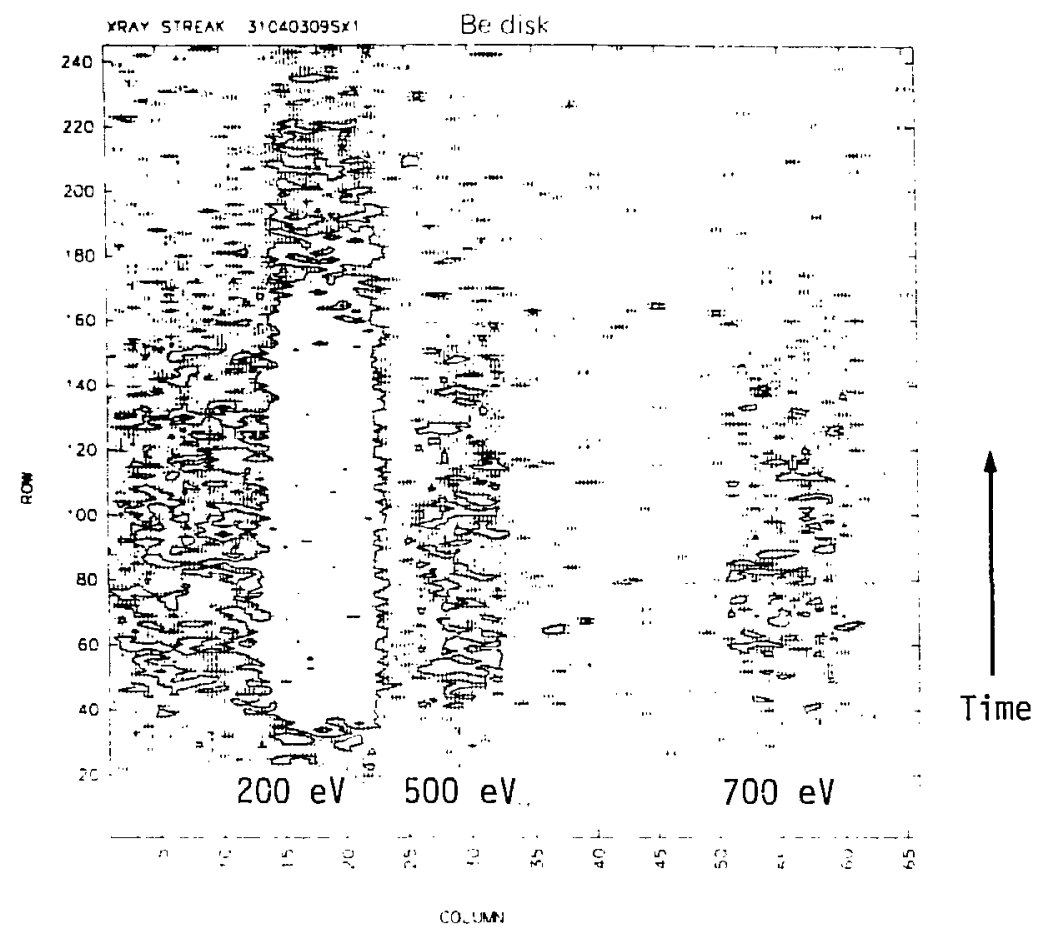

Figure A-18. Converted iso-intensity contours from figure A-17. 


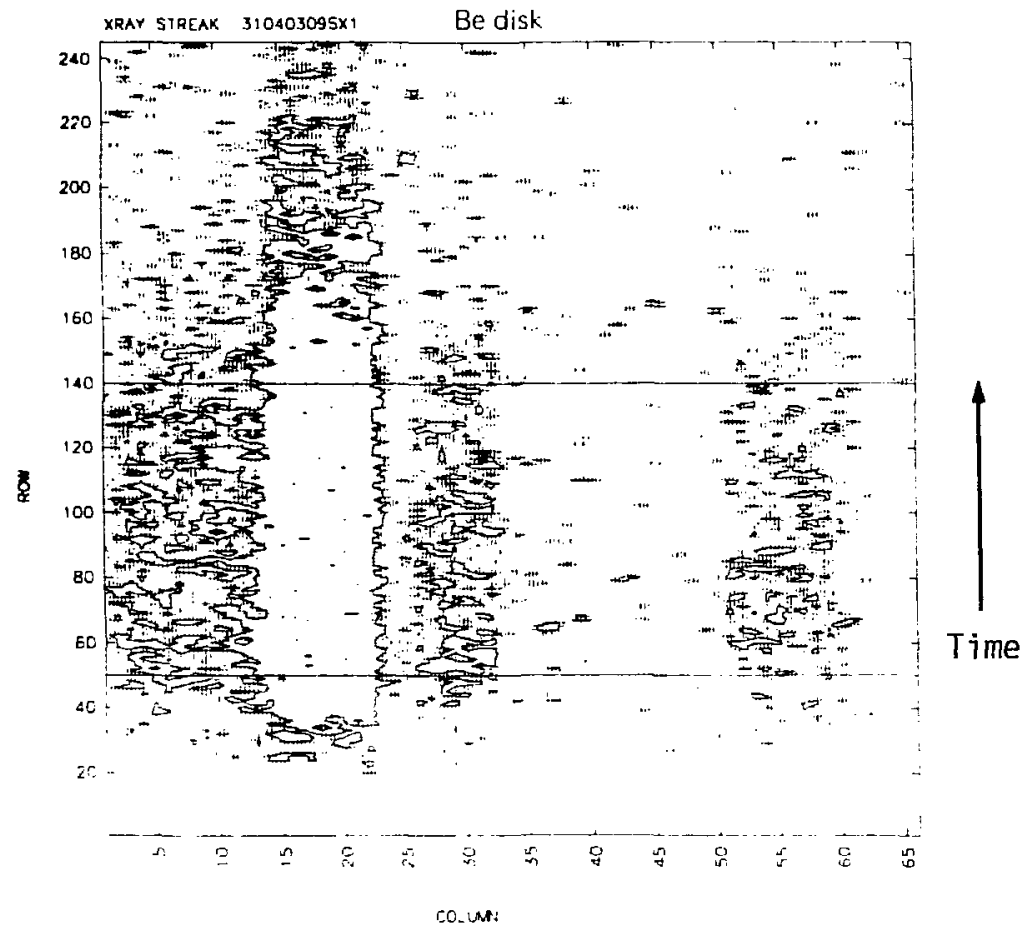

Figure A-19. Iso-intensity contour plot marked for temporal averaging over rows 50 through 140 . 


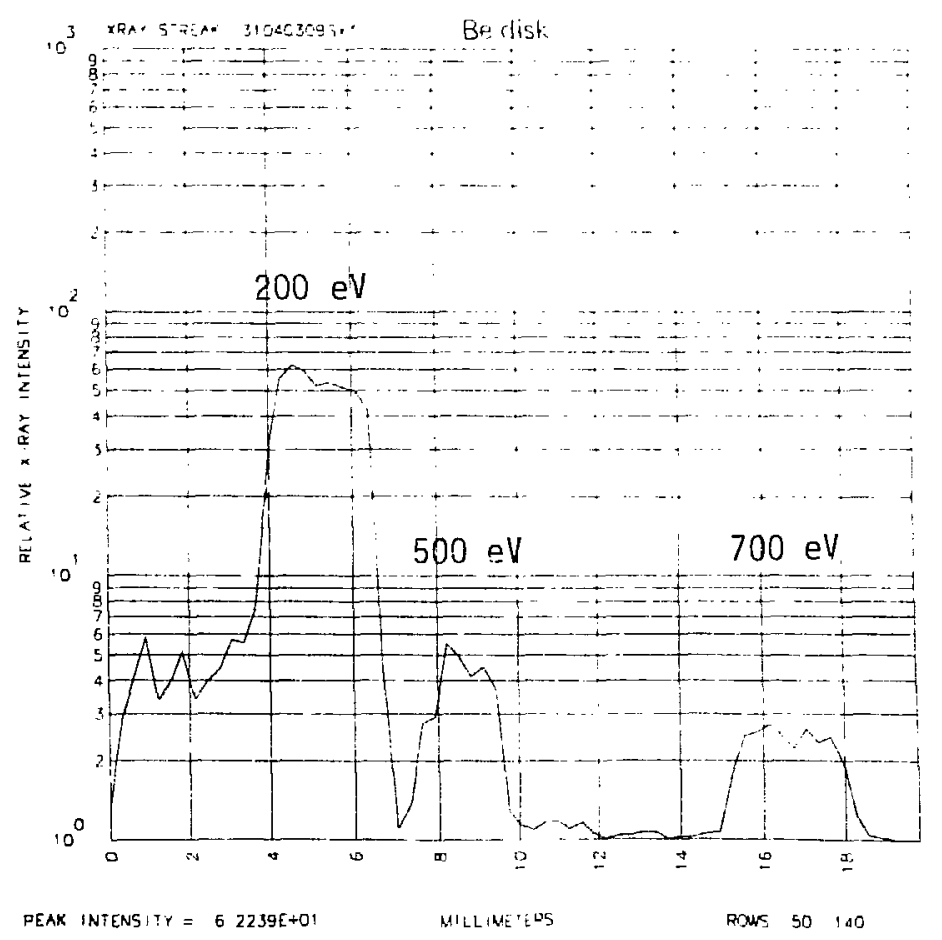

Figure A-20. Photocathode-position dependent, temporally-averaged channel levels. Low, signal levels result from the averaging of very-spikey, noisy data. 


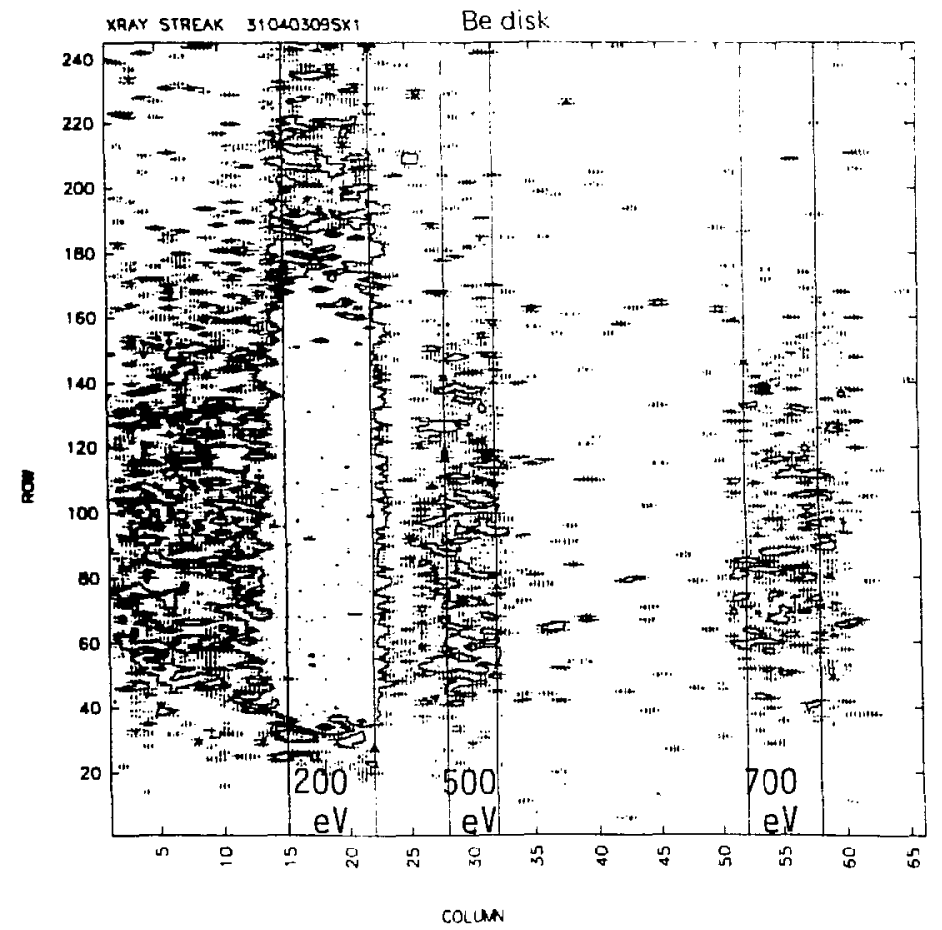

Figure A-21. Iso-intensity contour plot marked for spatial averaging in three channels. 


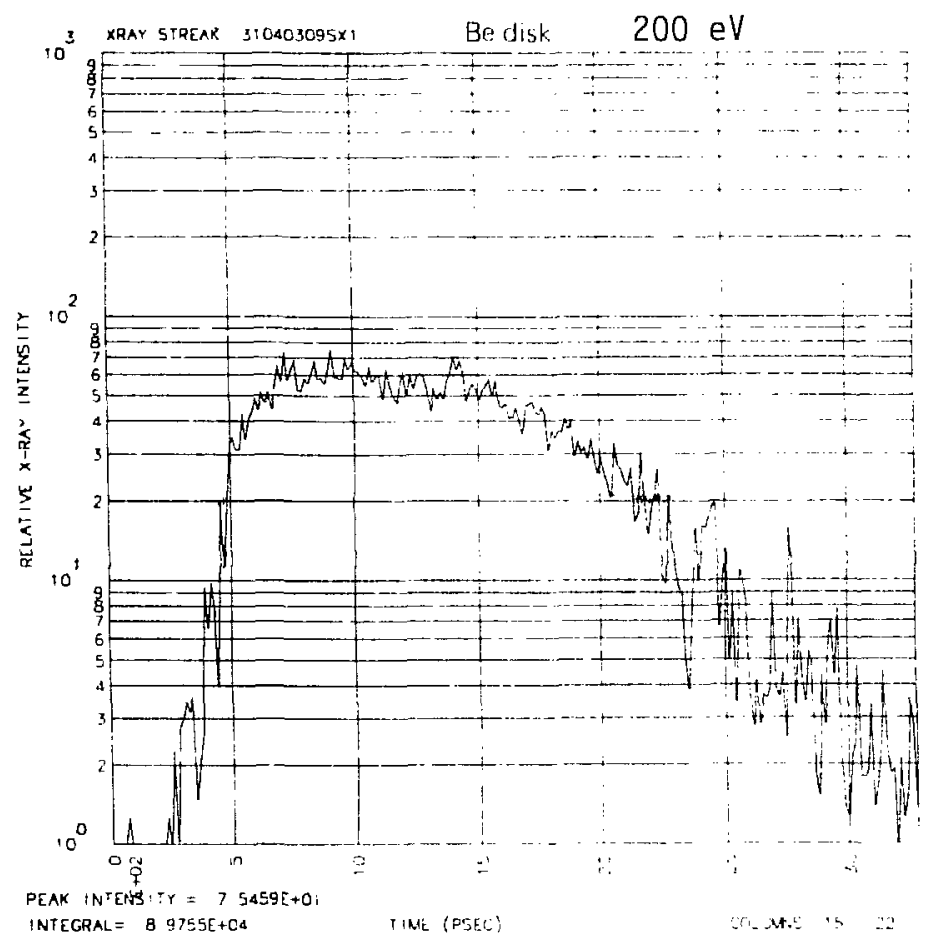

Figure A-22. Spatially averaged 200-eV (carbon filtered) channel as a function of time. Noise superimposed on the pulse shape hampers interpretation. 


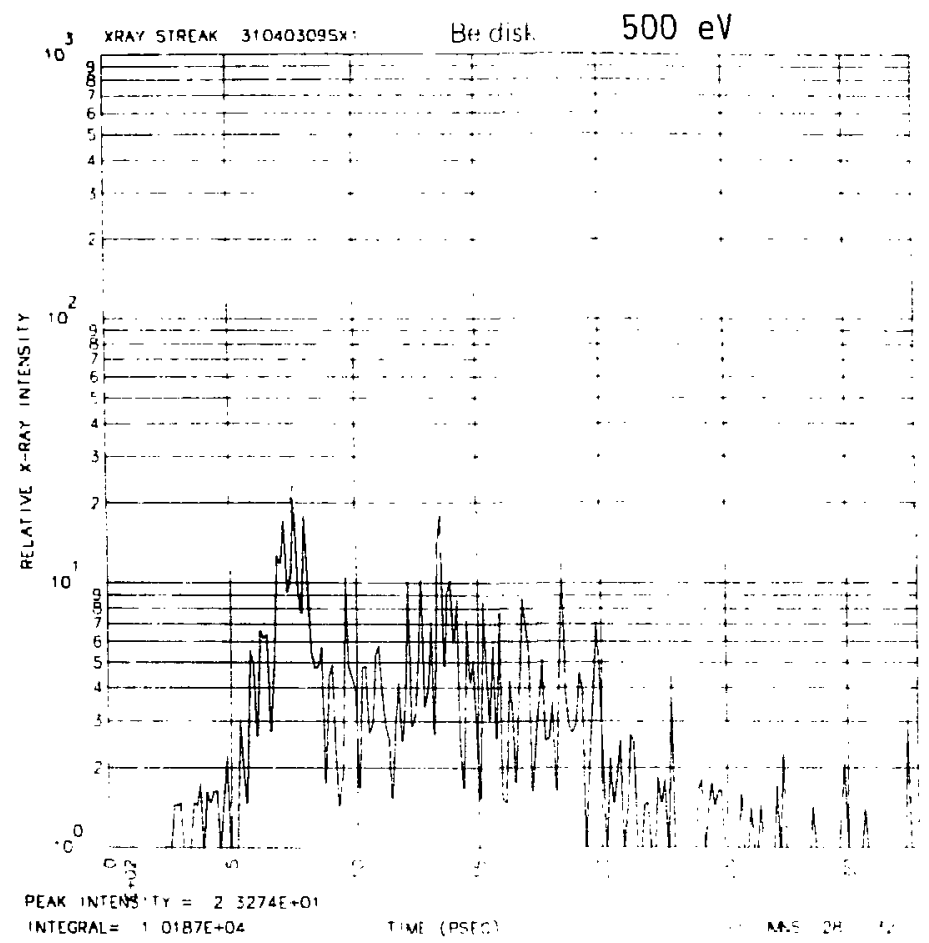

Figure A-23. Spatially-averaged 500-eV (vanadium filtered) channel. Data is too noisy for meaningful temporal analysis. 


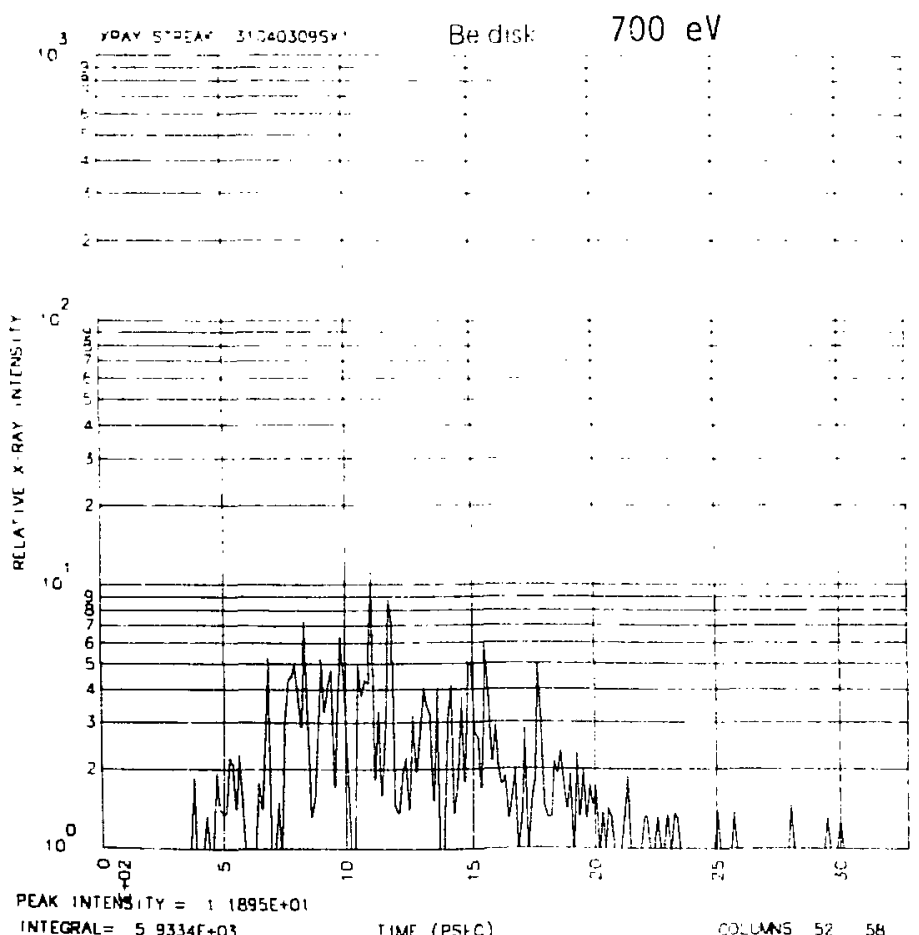

Figure A-24. Spatially-averaged 700-eV (iron filtered) channel. Data is too noisy for meaningful temporal analysis. 


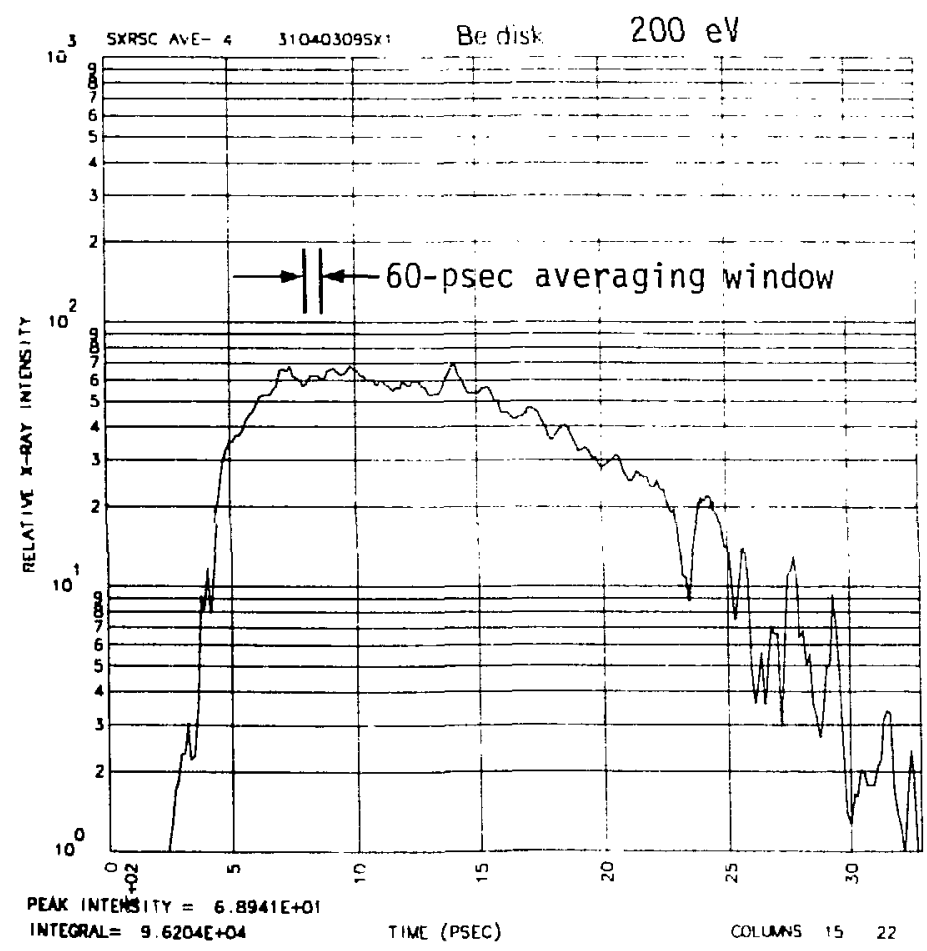

Figure A-25. The spatially-averaged 200-eV channel. Sixty picosecond temporal smoothing has been employed to suppress the noise. 


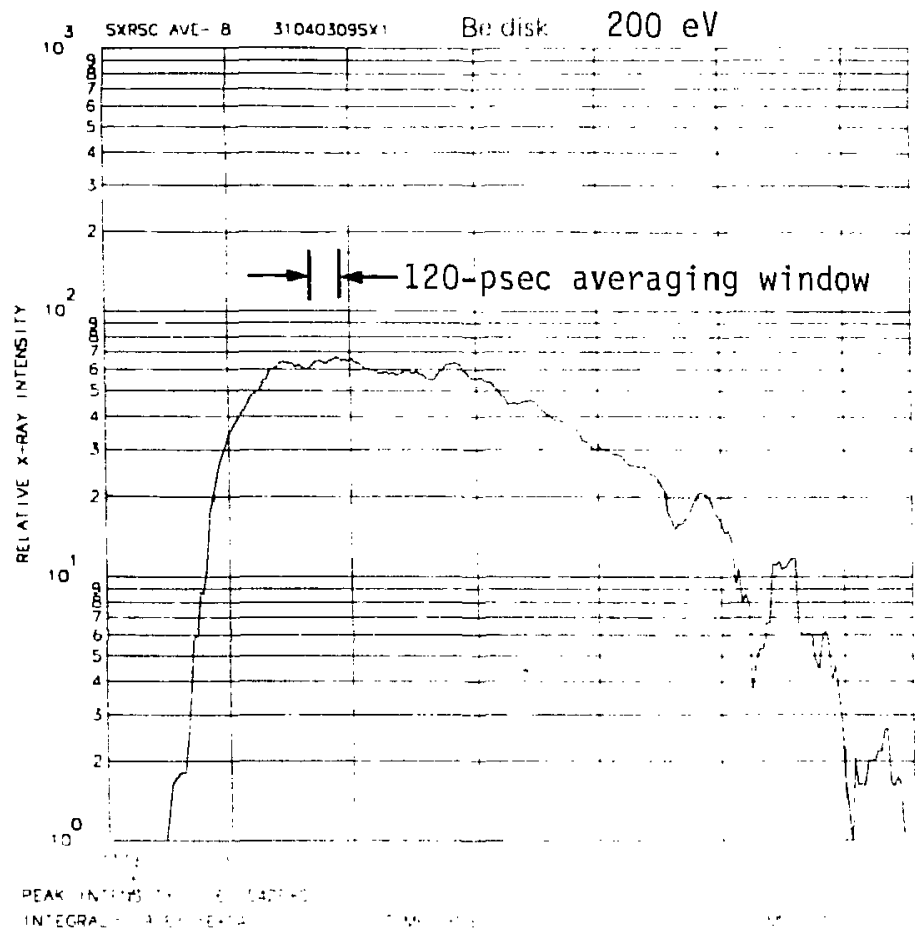

Figure A-26. The spatially-averaged 200-eV channel with 120-psec temporal smoothing. Small-scale noise has been eliminated and the signal shape is apparent. 


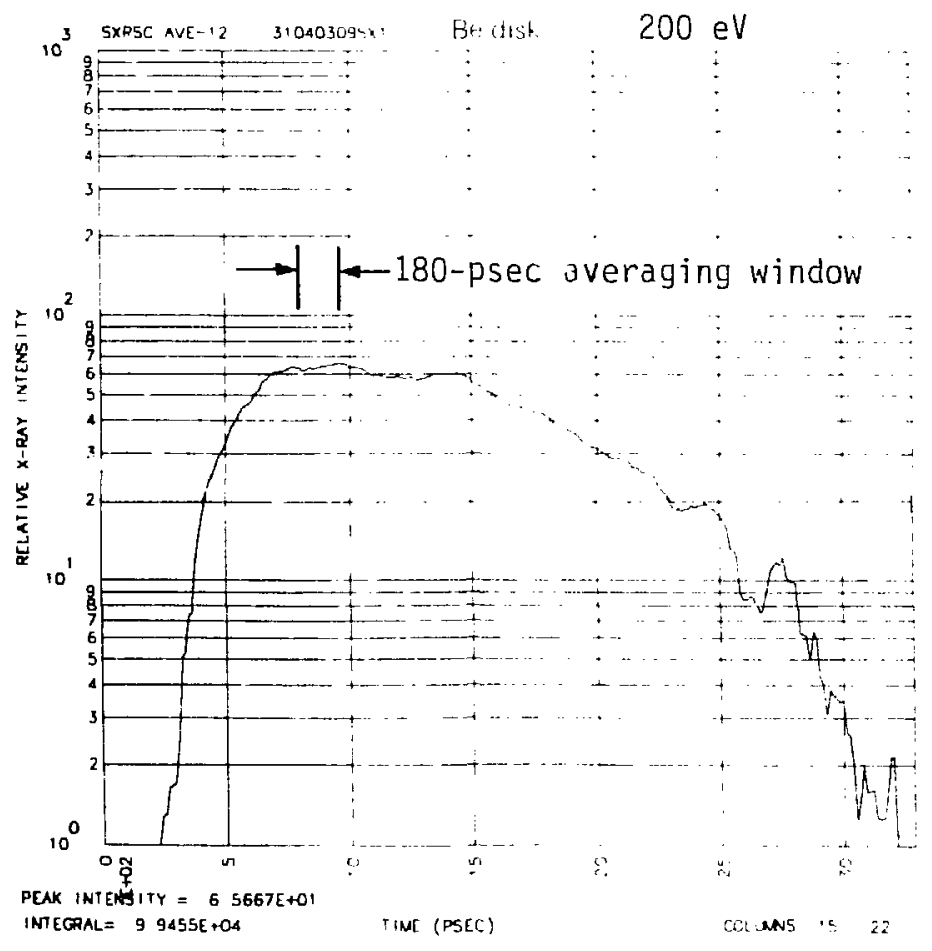

Figure A-27. The spatially-averaged 200-eV channel with 180-psec temporal smoothing. 